\title{
MASTER
}

ANL-CT-80-3

\section{DOE/ANL/HTRI HEAT EXCHANGER TUBE VIBRATION DATA BANK}

by

H. Halle, J. M. Chenoweth, and M. W. Wambsganss

Components Technology Division

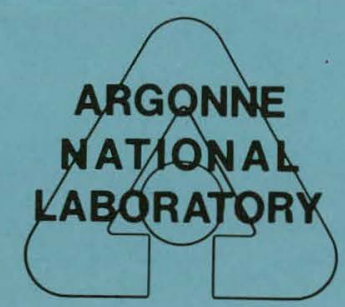

February 1980 


\section{DISCLAIMER}

This report was prepared as an account of work sponsored by an agency of the United States Government. Neither the United States Government nor any agency Thereof, nor any of their employees, makes any warranty, express or implied, or assumes any legal liability or responsibility for the accuracy, completeness, or usefulness of any information, apparatus, product, or process disclosed, or represents that its use would not infringe privately owned rights. Reference herein to any specific commercial product, process, or service by trade name, trademark, manufacturer, or otherwise does not necessarily constitute or imply its endorsement, recommendation, or favoring by the United States Government or any agency thereof. The views and opinions of authors expressed herein do not necessarily state or reflect those of the United States Government or any agency thereof. 


\section{DISCLAIMER}

Portions of this document may be illegible in electronic image products. Images are produced from the best available original document. 
The facilities of Argonne National Laboratory are owned by the United States Government. Under the terms of a contract (W-31-109-Eng-38) among the U.S. Department of Energy, Argonne Universities Association and The University of Chicago, the University employs the staff and operates the Laboratory in accordance with policies and programs formulated, approved and reviewed by the Association.

\section{MEMBERS OF ARGONNE UNIVERSITIES ASSOCIATION}

The University of Arizona

Carnegie-Mellon University

Case Western Reserve University

The University of Chicago

University of Cincinnati

Illinois Institute of Technology

University of Illinois

Indiana University

The University of Iowa

Iowa State University
The University of Kansas

Kansas State University

Loyola University of Chicago

Marquette University

The University of Michigan

Michigan State University

University of Minnesota

University of Missnuri

Northwestern University

University of Notre Dame
The Ohio State University

Ohio University

The Pennsylvania State University

Purdue University

Saint Louis University

Southern Illinois University

The University of Texas at Austin

Washington IIniversity

Wayne State University

The University of Wisconsin-Madison

\section{NOTICE}

This report was prepared as an account of work sponsored by an agency of the United States Government. Neither the United States Government or any agency thereof, nor any of their employees, make any warranty, express or implied, or assume any legal liability or responsibility for the accuracy, completeness, or usefulness of any information, apparatus, product, or process disclosed, or represent that its use would not infringe privately owned rights. Reference herein to any specific commercial product, process, or service by trade name, mark, manufacturer, or otherwise, does not necessarily constitute or imply its endorsement, recommendation, or favoring by the United States Government or any agency thereof. The views and opinions of authors expressed herein do not necessarily state or reflect those of the United States Government or any agency thereof. 
ANL-CT-80-3

Engineering and

Equipment (UC-38)

DOE/ANL/HTRI HEAT EXCHANGER TUBE

VIBRATION DATA BANK

by

H. Halle, J. M. Chenoweth, and M. W. Wambsganss

Components Technology Division

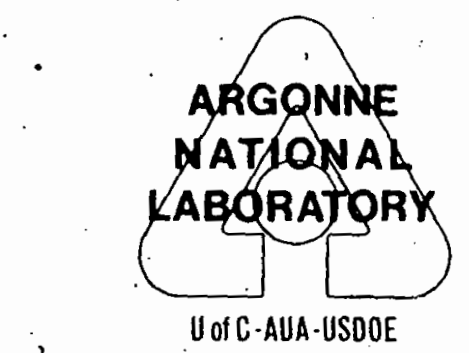

,

DISClaimer

This book was prepared as an occount of work sponsored oy en ogeney of itit Unitod Sintes Government. Neither the United States Government not any apency thereoil. nor any of their emploves. macasach. warraniy, express or implied, or asdumes any legal hatast product, or process disclosed, or

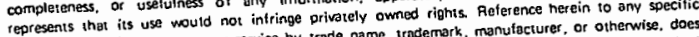
commercial product, process, or service by rade name, racemmendation, or thworing by the United not nesessarily constivis or imply hrot. The views and opinions of authors expressed herein do not any agency theteof.

February 1980

\footnotetext{
*Assistant Technical Director, Heat Transfer Research, Inc., Alhambra, CA
} 
I. INTRODUCTION :

II. BACKGROUND :

III. OBJECTIVES + . . . . 5

IV. ASSESSMENT OF DIFFICULTIES

V. DATA FORM

VI. SOLICITATION . $\quad \ldots \quad \ldots \quad \ldots \quad 7$

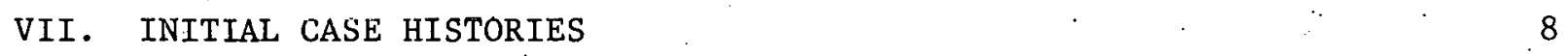

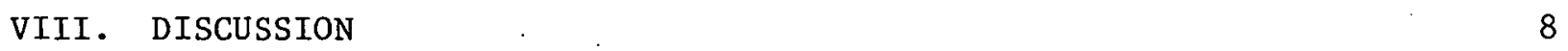

APPENDIX 1: HTRI Solicitation Letter for Tube Vibration Case Histories 1-1 and DOE/AIJL/HTRI Heat Exchanger Tube Vibration Data Form 
DOE/ANL/HTRI . HEAT EXCHANGER TUBE VIBRATION DATA BANK

by

H. Halle, J. M. Chenoweth, and M. W. Wambsganss

ABSTRACT

Development of a new heat exchanger tube vibration data bank at Argonne National Laboratory is described. The objective is to accumulate comprehensive case histories on heat exchangers that have experienced tube-vibration problems and units that have been trouble-free, and render this information available for evaluation, improvement, and development of vibration-prediction methods and design guidelines. Discussions include difficulties in generating a data bank, data form development, and solicitation efforts. Also included are 15 case histories upon which the data bank will be built. As new case histories are received, they will be asseinbled and published as addenda to this report.

\section{INTRODUCTION}

Flow-induced vibration has become a vital factor in the design of heat exchangers along with the more traditional thermal, hydraulic, and mechanical considerations. There have been many cases of shell-and-tube heat exchangers experiencing vibration problems, often leading to tube failures, and, in some cases, costly plant shutdowns. These vibration problems have motivated theoretical and experimental studies which have resulted in the development of prediction methods and design guidelines to avoid detrimental tube vịbration. However, most of these studies involved single tubes or tube banks subjected to idealized uniform crossflow or parallel flow conditions. Although these conditions can occur locally, they are not $\therefore$ 
typical of the changing flow pattern's throughout an actual she11-and-tube exchanger: Flow passages, and thus flow conditions, are much more complex than simulated by either mathematical models or experimental equivalents. Consequently, extrapolation of these methods for predicting flow-induced vibration problems to heat exchanger tube bundles has been seriously challenged. Available prediction methods provide useful guidance but are considered unreliable to the extent that their application to heat exchanger design evaluation is uncertain thus requiring the use of large factors of safety.

To examine the ability of these methods to correctly predict the absence ur presence of flow-induced tube vibrations, fleld data must be collected from case histories of individual heat exchangers and stored in a manner that can be input into these methods.

Efforts in support of such a data bank are being carried out at Argonne National Laboratory' (ANL), and funded by the U.S. Department of Energy, Office of Fossil Energy,.Division of Fossil Fuel Ittilizatinn. The activity io also a part of a U.S. contribution to an International Energy Agency (IEA) program of Research and Development on Heat Transfer and Heat Exchangers.

Solicitation and collection of case histories for the data bank has been assigned by ANL to Heat Transfer Research, Inc. (HTRI), a nonprofit cooperative of heat transfer. equipment manufacturers, engineering contractors, and processing companies to promote application-oriented research in heat transfer. ANL is working with HTRI to take advantage of their experience with data banks, their established contacts with manufacturers and users throughout the world, and to ensure anonymity of contributors to the bank.

This report describes the DOE/ANL/HTRI Heat Exchanger Tube Vibration Data Bank, including background information, objectives, assessment of difficulties involved, data form development, solicitation efforts, and, as an appendix, fifteen case histories of heat exchanger tube vibration. These histories were purchased 
from HTRI with program funds and represent cases in which acoustic vibration, damaging tube vibration, and no vibration were present. They constitute the initial entries and the base upon which the new data bank will be built. As additional case histories are received, the data will be collated and published annually as addenda to. this report.

\section{BACKGROUND}

The need for a bank of field data for she11-and-tube heat exchargers that have experienced flow-induced tube vibration problems was recognized by the Tubular Exchanger Manufacturers Association (TEMA) in 1969. Data collected from its members represented observations of gross effects that were considered significant parameters concerning tube vibrations. These proprietary data were made available to HTRI in 1971. Since that time, a new understanding of vibration phenomena has shown that the data are inadequate to evaluate critically current prediction methods.

In 1972, HTRI started collecting case histories for a heat exchanger tube vibration data bank. A form was designed that requested more data than found in the TEMA Data Bank. More specifically, case histories also were requested for heat exchangers which had not experienced vibration problems.

The data obtained ranged from very complete to sparse. In some instances, data gaps could be filled either by contacting the organization submitting the case, by making back-calculations, or by using "good engineering judgment." Although the HTRI data bank is proprietary to HTRI members, gross comparisons of predicliouns by known methods with fiel.d observations have been published. ${ }^{\dagger}$ of the more than 60 cases submitted, only 25 were sufficiently documented to

\footnotetext{
†J. M. Chenoweth, "Flow-Induced Tube Vibrations in Shell and Tube Heat Exchangers," ERDA Report SAN/1273-1 (February 1977).
} 
be included in the data bank. However, only six of these cases deal with liquid shellside flow. Additionally, the data' are heavily biased toward cases for which vibration problems have been experienced; there is a lack of cases for which no vibration problems occurred.

In England, Heat Transfer and Fluid Flow Service (HTFS) also has collected, analyzed, and prepared a report on tube-vibration data. However, the report is proprietary to HTFS members.

In 1976, HTRI conducted a study on flow-induced tube vibration in she11and-tube heat exchangers for the Division of Conservation Research and Technology of the Energy Research and Development Administration (ERDA). This study included a Heat Exchanger Tube Vibration Workshop to identify the most promising areas of needed research in flow-induced vibration in industrial shell-and-tube heat exchangers. An international panel of 14 vibration experts, representing ongoing research, was invited to present their evaluation of the current stateof-the-art, and to participate with other attendees in discusciono and formulation of research recommendations. The results of the presentations and discussions were published by Chenoweth.

During the workshop, the panelists stressed the importance of obtaining and using field data to establish the validity of any prediction method. The difflculty of obtaining reliable field data was recognized, and the development of improved methods (i.e.., dața banks) to acquire such data was recommended.

Based, in part, on the results and recommendations from this workshop, development of a bank of field data was selected as one of two program activities to be assigned to ANL. The second activity involves obtaining tubevibration data under controlled conditions from testing of a specially built, industrial size; segmentally baffled shell-and-tube heat exchanger. 


\section{OBJECTIVES}

The DOE/ANL/HTRI Heat Exchanger Tube Vibration Data Bank has as its immediate objective the collection of sufficient, reliable, well-documented field data to (1) demonstrate confidence in the application of available prediction methods to the design of equipment; (2) provide a basis for improving these methods; or (3) provide the researcher with information for developing new methods. The ultimate goal, in addition to reducing the number of heat exchangers that experience detrimental vibrations, is to minimize the number of heat exchangers that are unnecessarily overdesigned to avoid flow-induced vibration problems.

\section{ASSESSMENT OF DIFFICULTIES}

Following are some of the reasons why it may be difficult to obtain comprehensive field data on flow-induced vibration in shell-and-tube heat exchangers.

(1) Plant managers and field service engineers are, understandably, more interested in getting a plant back into production than investigating the source of vibration-induced failure of a heat exchanger.

(2) Before a vibration problem develops, there is no incentive to make observations that could identify the source of the problem. Thus, although a long-term phenomenon may have caused the failure, recent operational changes are usually suspected.

(3) Actual operating conditions of ten do not agree with conditions assumed in the design of a heat exchanger, particularly during startup, shutdown, and plant upsets. It is reasonable to suspect many vibration problems are initiated during these phases of operation. 
(4) Flow velocity profiles within a tube bundle and tube damping, both important parameters in any prediction method, are difficult to measure and to calculate.

(5) Records are seldom kept of "as built" heat exchangers. Deviations during initial construction and subsequent field changes are not always noted. on drawings.

(6) Before organizations will release the desired amount of data, they must be assured that its source will be protected and that its use will be restricted to evaluation of prediction methods.

The last item was a contributing factor in the decision to assign an independent, non-government organization (i.e., HTRI) the task of collecting, storing, and coding the data forwarded to ANL.

\section{DATA F FORM}

It is important that the data be collected in a systematic way, be as complete as possible, and as accurate as practical.' Toward this end, a standard questionnaire is required which lists the desired information that may be available but may not be considered significant by each, contributor. The questionnaire should provide entries for sufficient primary data to enable an investigator (1) to calculate important derived data such as flow velocity fractions and natural frequencies (if not measured); (2) to input the data in various predition methods; (3) to compare the calculated results with actual performance; and (4) to request additional information that may. be made available by the source. 
The DOE/ANL/HTRI Heat Exchanger Tube Vibration Data Form and Instruction Sheet developed to satisfy these requirements are reproduced in Appendix 1. The 4-page data form is based on a Heat Exchanger Vibration Data Sheet developed and used by HTRI to accumulate their data bank. In consultation with HTRI, the format of their data sheet has been rearranged and enlarged to motivate and accommodate more input, particularly with respect to vibration damage description, analysis of two-phase flow, and evaluation of the influence of axial forces on tubes.

As the case histories are received by HTRI, they will be coded. Page 1 of the Data Form will be retained in a confidential file. Pages 2, 3, and 4, which contain the pertinent technical information will be sent to ANL for inclusion in the DOE/ANL/HTRI Heat Exchanger Tube Vibration Bank.

VI. SOLICITATION

As discussed above, the task of soliciting case histories has been assigned to HTRI. The letter of solicitation that accompanies the data form is included in Appendix 1.

A direct solicitation for case histories has been made to HTRI member organizations (> 150) located through the world. Additionally, non-HTRI member organizations will be solicited.

The vibration problem is sufficiently complex, that a follow-up procedure will. be implemented to ensure complete documentation of each case history. In addition, the quality of the information will be evaluated; poor data with inadequate documentation will only be misleading. It is better to have fewer. reliable data in a data bank than a larger amount of questionable data. 


\section{INITIAL CASE HISTORIES}

Fifteen of the most representative of we11-documented case histories have been purchased from the HTRI Data Bank, and the information transferred to the new data forms. These forms are reproduced as Appendix 2, and represent the initial case histories of the DOE/ANL/HTR.I Heat Exchanger Tube Vibration Data Bank. Owing to the enlarged format and increased number of entries, there are areas where the data are incomplete.

Also included in Appendix 2 is a summary tabulation of the geometries and various process fluids of the heat exchangers.

\section{DISCUSSION}

Successful development of a data bank of heat exchanger tube vibration case histories cannot be guaranteed a priori because of the many difficulties in obtaining data that are sufficiently detailed and reliable. For the most part, these difficulties are beyond the control of the data bank developer. In fact, some investigators who have attempted to assemble a similar bank for evaluation of prediction methods have concluded that such an effort, while, initially appealing, is difficult to accomplish in practice because of inherent uncertainties in the data.

Nevertheless, development of the DOF/ANI/HTRT Heat Exchanger Tubs Vibration Data Bank is deemed a significant effort toward a better understanding of the phenomena involved, and marked improvement in formulation and application of prediction methods. .

More speclfically: Although information in the data bank will be predominantly of a qualitative nature, it will aid researchers and design engineers in (1) identifying regions within a tube bundle (such as the first row after the baffle cut) that are most susceptible to vibration and whether 
the mode of failure is tube wear at the baffle or intertube impacting; and (2) determining the relative effectiveness of various design features (e.g., impingement plates) in reducing the potential for tube vibration.

As a source of practical information, the data bank will be particularly beneficial to researchers who have no direct experience in design or operation of heat exchangers, but are involved in development of design guidelines and corresponding performance-prediction methods.

For the effort to be successful will require the cooperation of the contributors to the bank to ensure that complete and accurate data are provided. Also, it will be necessary to collect case histories from heat exchangers that have as we 11 as those that have not experienced vibration problems, and from units with liquids, gases, and two-phase mixtures as the shellside fluid. As the number of respective case histories increases, trends in the dynamic behavior will become apparent, as will any anomalies that should be disregarded because of inaccurate or uncertain data. As necessary, selected case histories will be followed up with personal contacts and/or on-site visits for purposes of obtaining clarification or additional information.

It is not unreasonable to expect that it will take several years to collect a sufficient number of we11-documented case's for the data bank to become a useful tool in the evaluation of prediction methods. However, during this time period the available data will provide useful guidance of research efforts. For example, new methods to calculate flow velocities may be derived which will increase the potential usefulness of the data over what it would be today. 
APPENDIX 1

HTRI Solicitation Letter for Tube Vibration Case Histories

and

DOE/ANL/HTRI Heat Exchanger Tube Vibration Data Form 
Subject: Tube Vibration Data Bank on Shell-and-Tube Heat Exchangers

The importance of correctly predicting, and thus eliminating, the danger of possible tube vibration in shell-and-tube heat exchangers hardly needs to be emphasized. There are many known cases where tube vibration resulted in destruction of tubes with very dangerous and costly consequences. HTRI has been active in tube vibration research for the past eight yejars.

In October 1976 HTRI organized under contract to ERDA (presently US Department of Energy, DOE) a Tube Vibration Workshop with a panel discussion on the state of the art by selected international experts. (Reference ERDA Report No. SAN/1273-1.) One of the principal conclusions was the urgent need for a tube vibration data bank. To test the various predictive methods developed from theory or laboratory studies, field data on large-scale industrial shell-and-tube heat exchangers are necessary. Our subsequent contract with DOE resulted in

- HTRI consulting on a research program involving a large-scale test exchanger for vibration study at the Argonne National Laboratory. This test is currently under way and is producing most important data. The results will eventually be published as a report from Argonne National Laboratory and will also be made available to the International Encrgy Agency.

- HTRI, acting on bèhalf of LUE/Argonne, soliciting and collecting data for a new DOE/ANL/HTRI Heat Exchanger Tube Vibration Data Bank.

The reason Argonne National Laboratory has given the task of establishing the Tube Vibration Data Bank to HTRI is (a) our previous experience in the proper assembly of tube vibration data, and (b) to assure anonymity of the data sources.

By this letter I am requesting support from your company to supply field data on shell-and-tube heat exchangers that experienced vibration problems as well as data on similar exchangers that did not experience vibration problems. Such cases to be useful need to be reasonably well documented. A new Data Form (attached) was developed to assist in collecting the data. It reflects our experience and anticipates items that are significant but might be overlooked. The identity of the data source will be confldential to H'I'KI Statt as only pages 2, 3, and 4 of the Data Form will be included in the Data Bank. Your cooperation will assure that badly needed data will be available for a well-organized international cooperative project on flow-induced vibration and ultimately the formulation of improved methods for tube vibration prediction.

Sincerely yours,

JT:pth

Attachment
Jerry Taborek

Technical Director 


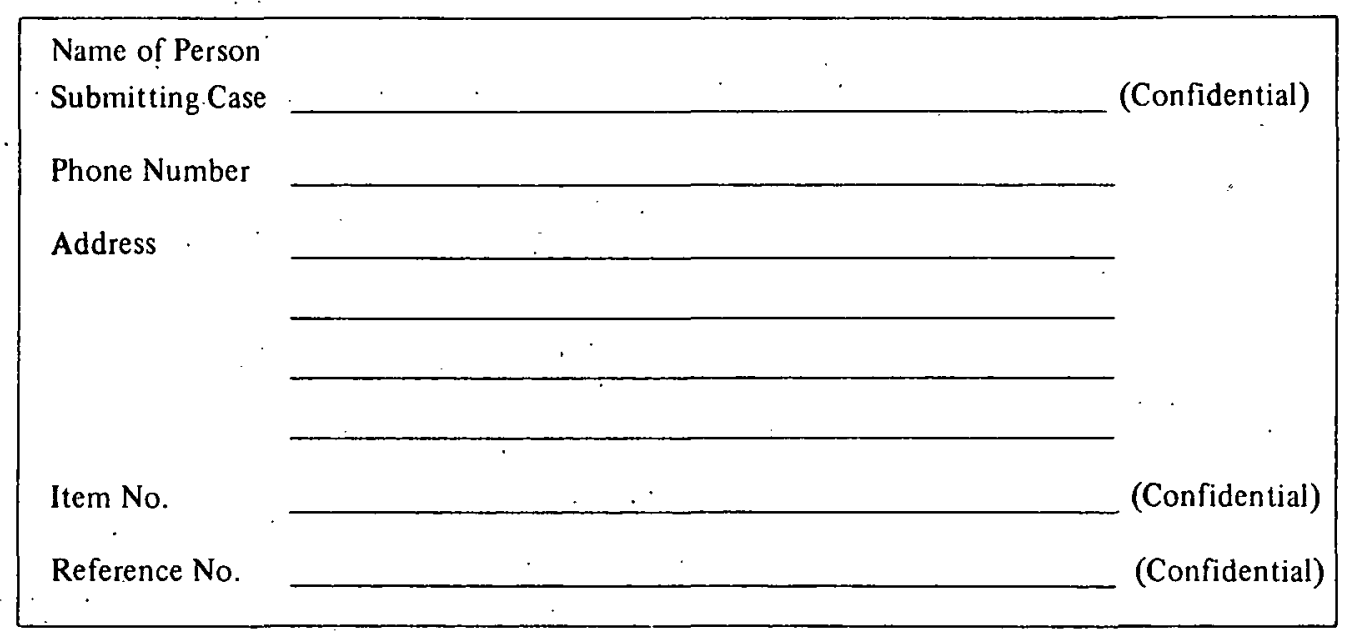

Assigned

Case No.
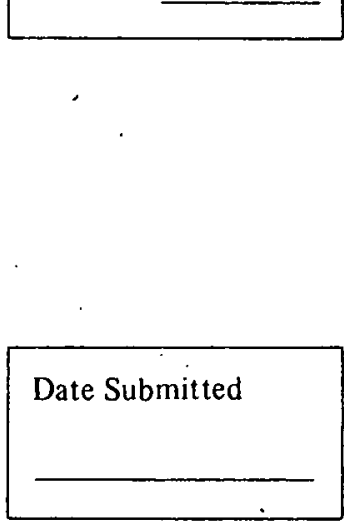

\section{INSTRUCTIONS}

HTRI is collecting data on vibration and vibration damage in shell-and-tube heat exchangers which will be incorporated into a Vibration Data Bank sponsored by the U. S. Department of Energy (DOE). Some years ago, TEMA and HTRI collected case studies of vibration which have become the initial entries in the data bank. Recognizing that more comprehensive data are needed, this data collection form has been prepared. The case studies will be used to test and to aid in the improvement of proposed correlations for predicting the occurrence of vibration and possible damage which might result. Until a sufficiently broad data bank can be assembled and used to test possible prediction methods, it will be impossible to make predictions with any degree of confidence.

Any predictive method must not only indicate when vibration problems are probable, but must also predict accurately when they will be absent. Consequently, we are interested in also receiving a number of cases of heat exchangers which did not experience vibration and yet were similar to ones which had vibration problems. Much of the requested information may be available from drawings and/or specification sheets for the exchangers. If these are supplied, only the flow conditions and observed descriptions of damage, vibration frequencies, etc. need be filled in on the data sheets. The company submitting the data and any Item and Reference Numbers used to identify the exchanger will be confidential. HTRI will assign a case number and forward the data to Argonne National Laboratory (ANL) for publication for DOE.

Plcasc fill in the fornis as completely as possible. Supplement with photographs, sketches, drawings, and descriptions if available. Indicate known deviation from construction drawings. Add anything you fecl would assist in understanding flow-induced vibration. For the benefil of all, please contribute as many cases as possible. Mail the completed forims with attachments to:

Heat Transfer Research, Inc. $1000 \mathrm{~S}$. Fremont Avenue

Alhambra, CA 91802 
Type $\square$ TI:MA Exchanger Designation

Special Exchanger (Describe)

SHELL GEOMETRY (Complete Sketch Below)

Inside Diameter, mm (in.)

Wall Thickness, mm (in.) Material

Inlet Nozzle ID, mm (in.)

Outlet No\%lc ID, mm (in.)

Impingement Protection $\square$ No $\square$ Yes

(Describe)

Annular Distributur $\square$ PNu a'res

Open Cut Area $\mathrm{mm}^{2}$ (in. ${ }^{2}$ )

Nozzle-to-First Tube Row Distance, mm (in.)

\section{CROSS BAFFLE GEOMETRY}

Type $\square$ Segınental; $\square$ Double-Segmental $\square$ Disc/Doughnut

口 Triple-Segmental; $\square$ No=Tubes-in-Window

Baffle Cut, \% Shell Diameter

Cut Orientation Relative to Axis of Inlet Nozzle

Inlet Baffle $\square$ Perpendicular $\square$ Parallel $\square 45^{\circ}$

Central Baffles $\square$ Perpendicular $\square$ Parallel $\square 45^{\circ}$

Baffle Thickness, mm (in.) ___ Material

Diametral Clearances Shell-to-baffle, mm (in.)

Tubc-to-bafflc mm (in.)

Bundle-to-shell, mm (in.)

Number of Baftles Along Length of Shell

Baffle Spacing, $\mathrm{mm}$ (in.)

Central

Inlet Outlet

Unsupported Tube Span Lengths, mm (in.)

Longest

Inlet

Outlet
Shell Orientation " $\square$ Vertical

\section{TUBE GEOMETRY}

Outside Diameter, mm (in.)

Wall Thickness, mm (in.) Material

Tubc Lengths

Straiglıt Tube, Inside Tubeslieets, mun (in.)

U.T'ube, Tubesheet to Bend Tangent, IIIII (in.)

Tube Pitch, mm (in.)

Layout (Please Cirilè)

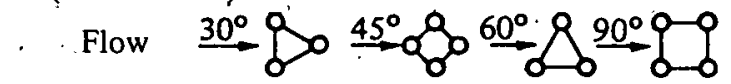

No. of Tubes

No. of Tubepasses

First Tubepass $\square$ Countercurrent $\square$ Cocurrent

Tube-to-Tubesheet Joint

$\square$ Welded $\square$ Roller Expanded $\square$ Other

If U-Tube

Maximum Bend Radius, mm (in.)

Bend Orientation Relative to Axis of Shellside Inlet Nozzle

$\square$ Perpendicular $\square$ Parallel

If Bend Supported, Describe in Comments Below

If Finned Tubes

Fins/m (Fins/in.) Fin Material

Diameter, mm (in.), Root Over Fins

If Limlanted Surface Tubes

(Describe)

\section{DETUNING BAFFLE}

If Detuning Baffle Used to Control Acoustic

Vihration, Indicate Position on Sketch Below

\section{COMMENTS AND SKETCH}

Complete sketches by drawing in tubeside and shelislde nozzles.

Indicate inlet nozzles with an arrow.
Show shellside Inlet nozzle location, baffle cut orientation, and impingement devices.
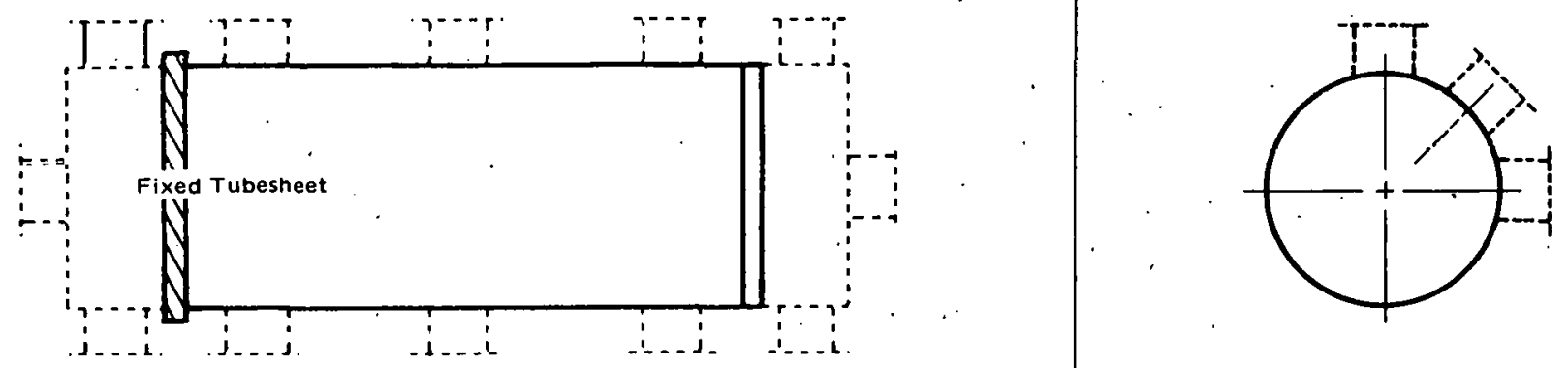

$\leftrightarrow$ Indicate top of exchanger as mounted 
Assigned

Case No.

PROCESS CONDITIONS

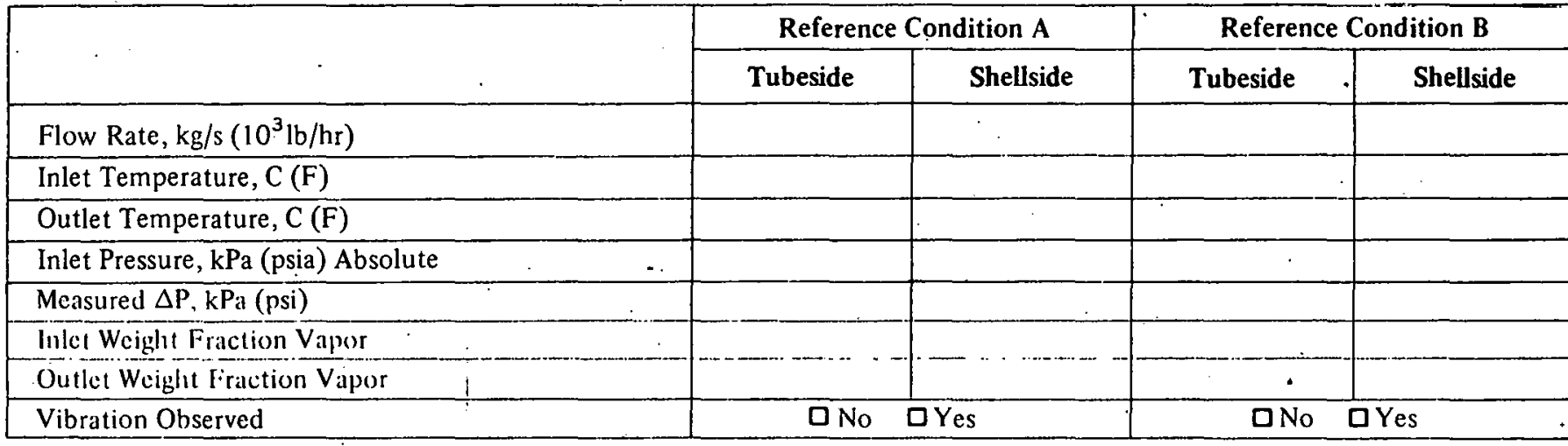

\section{FLUID PHYSICAL PROPERTIES}

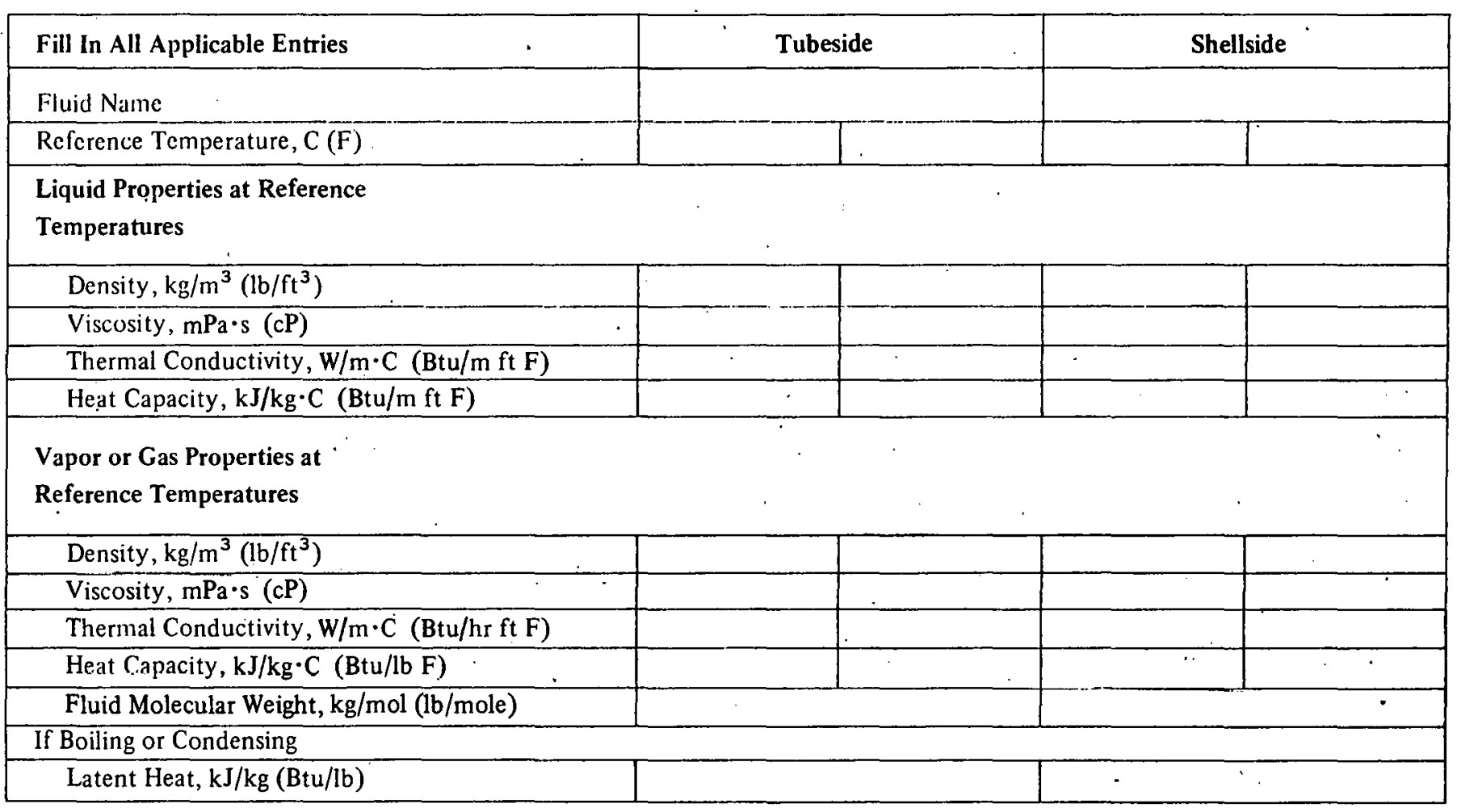


VIBRATION AND DAMAGE DESCRIPTION

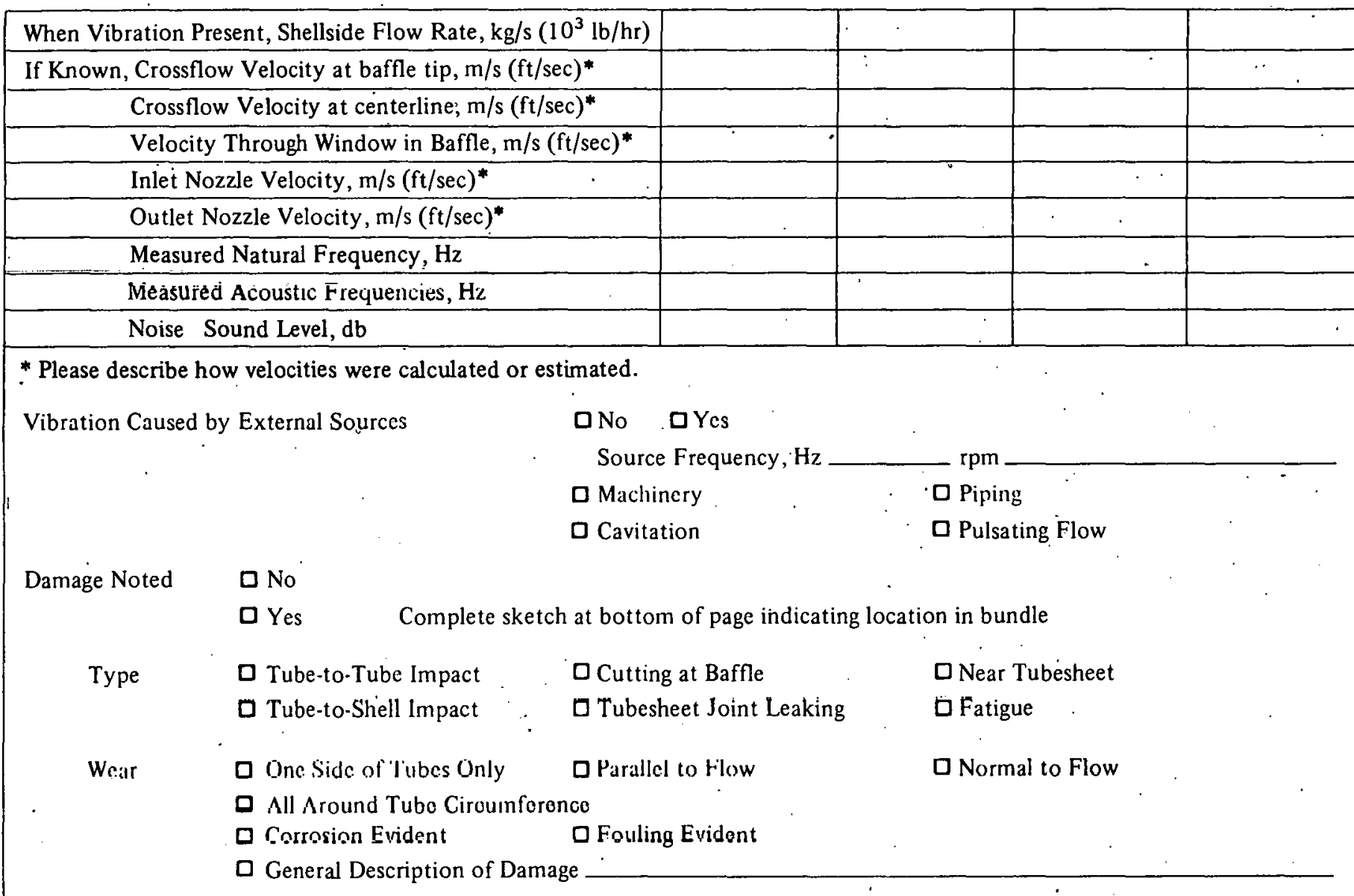

Exchanger Operation History

- How Long on Stream Before Damage Occurred?

- Any. Unusual Occurrence Observed Prior to Vibration as a Consequence of 口Start-up $\square$ Plant-Upset $\square$ Shutdown

Describe

- If Vibration Remedy Applied, Describe and Indicate Results:

\section{TUBE BUNDLE DAMAGE SKE ICH}
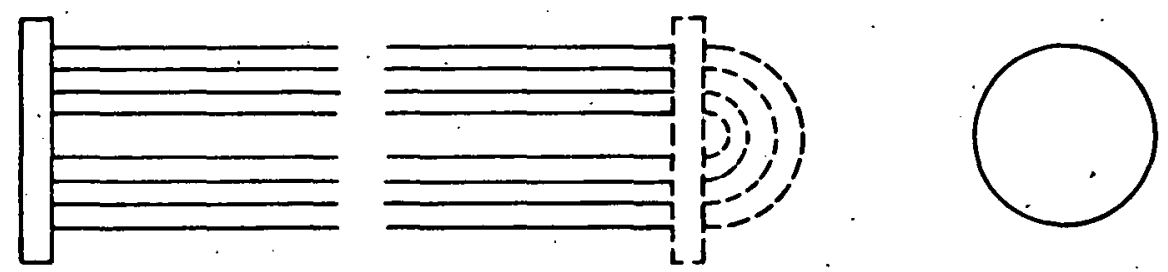
APPENDIX 2

Case Histories 101-115 of the DOE/ANL/HTRI Tube Vibration Data Bank 
Summary of Heat Exchanger Case Histories in Appendix 2

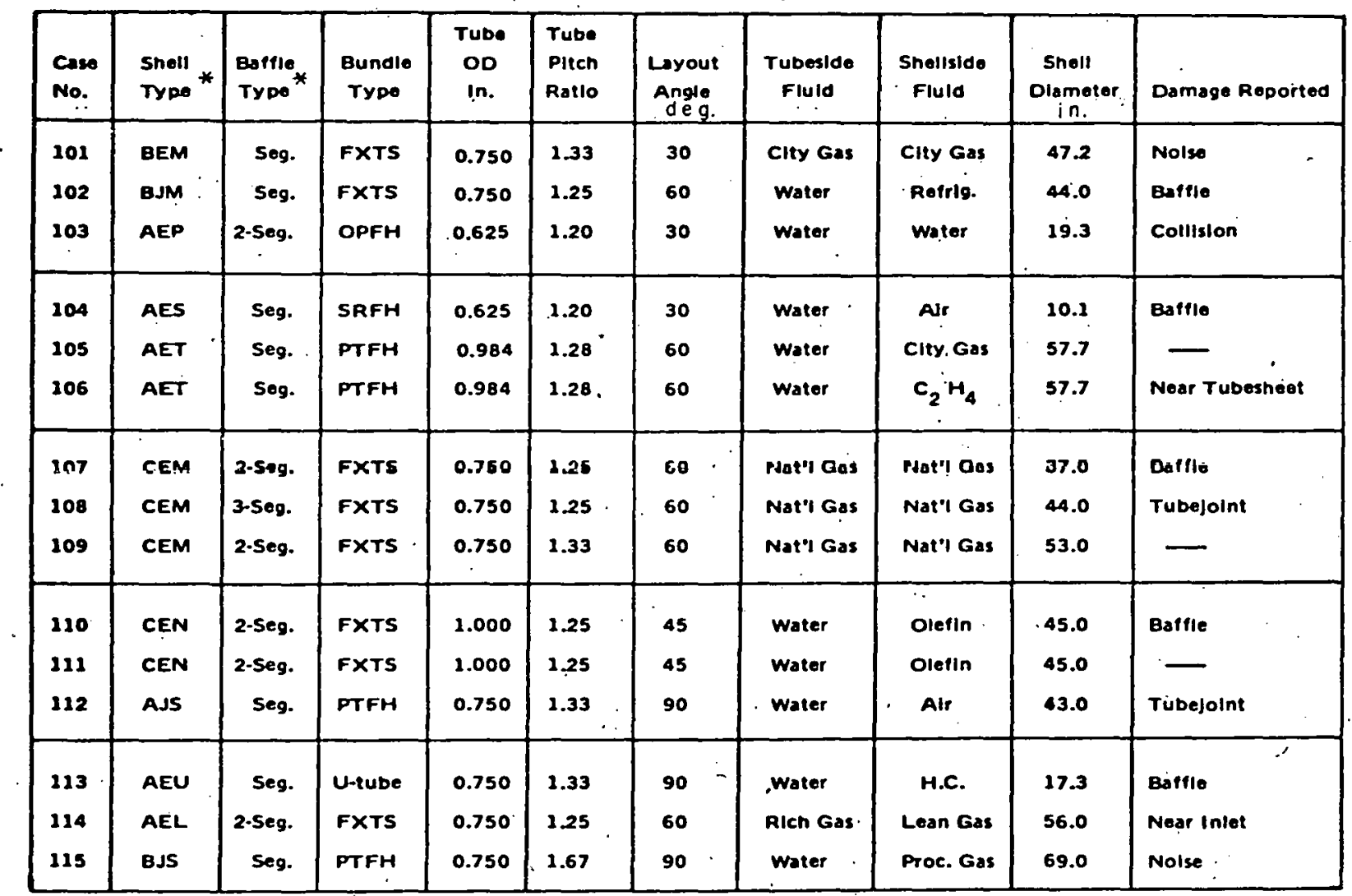

\footnotetext{
"For definitions, see Standards of Tubular Exchanger Manufacturers, Association, Sixth Edition, 1978.
} 
To protect the identity of the organization submitting this case, HTRI has assigned a case number. Additionally the data on pages 2, 3, and 4 have been reviewed to ensure that they do not include any propietary information.

This is a replacement for the original page 1 that provides space for Assigned Case No. additional comments, drawings, photographs, etc.

Summary

This 48-in. diameter by 13-ft long BEM TEMA style' heat exchanger with segmental baffles had "city gas" in both" the shell side and the tube side. Although no direct damage to the tubes was noted, there was a loud noise during. operation. The noise was assumed to have been the result of flow-induced acoustic vibration. Information is limited to that presented on data form. 


\begin{tabular}{|c|c|}
\hline & $\begin{array}{l}\text { Assigned } \\
\text { Case No. }\end{array}$ \\
\hline $\begin{array}{ccc}\text { Type } & \text { TEMA Exchanger } & \text { Designation BEM } \\
& \square \text { Special Exchanger } & \text { (Describe) } \\
\end{array}$ & $\begin{array}{l}\text { QHorizontal } \\
\text { DVertical }\end{array}$ \\
\hline SHELL GEOMETRY (Complete Sketch Below) & TUBE GEOMETRY \\
\hline $\begin{array}{l}\text { Inside Diameter, (in.) } 47.2 \\
\text { Wall Thickness, min (in.) } \\
\text { Inlet Nozzle ID, Material (in.) } 20 \\
\text { Outlet Nozzle ID, (in.) } 20\end{array}$ & 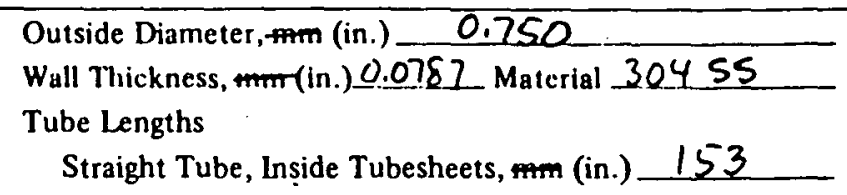 \\
\hline $\begin{array}{l}\text { Impingement Protection RNo } \square \text { Yes } \\
\text { (Describe) }\end{array}$ & $\begin{array}{l}\text { U.Tube, Tubesheet.to Bend Tangent, mm (in.) - } \\
\text { Tube Pitch, (in.) } \quad 1.00\end{array}$ \\
\hline 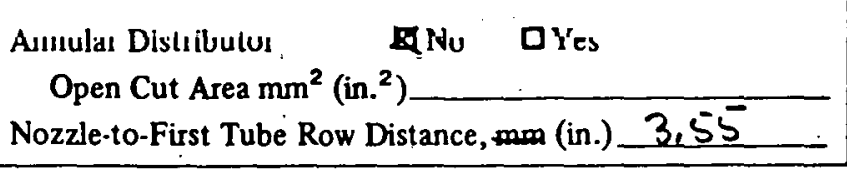 & Flow (30, 80$)$ No. \\
\hline CROSS BAFFLE GEOMETRY & First Tubepass $\square$ Countercurrent $\square$ Cocurrent \\
\hline $\begin{array}{l}\text { Type Segmental; } \square \text { Double-Segmental } \square \text { Disc/Doughnut } \\
\text { a Triple-Segmental; } \square \text { No-Tubes-in-Window } \\
\text { Baffle Cut, \% Shell Diameter } 25\end{array}$ & $\begin{array}{l}\text { Tube-to-Tubesheet Joint } \\
\text { ॠWelded } \square \text { Roller Expanded } \square \text { Other } \\
\text { If U-Tube } \\
\text { Maximum Bend Radius, mm (in.) }\end{array}$ \\
\hline $\begin{array}{l}\text { Cut Orientation Relative to Axis of Inlet Nozzle } \\
\text { Inlet Baffle Perpendicular } \square \text { Parallel } \square 45^{\circ} \\
\text { Central Baffles Perpendicular OParallel } \square 45^{\circ}\end{array}$ & $\begin{array}{l}\text { Bend Orientation Relative to Axis of Shellside Inlet Nozzle } \\
\text { Q Perpendicular D Parallel } \\
\text { If Bend Supported, Describe in Comments Below }\end{array}$ \\
\hline $\begin{array}{l}\text { Baffle Thickness, (in.) } 0.437 \text { Material } \frac{?}{\text { Diametral Clearances Shell-to-baffle, (in.) } 0.118} \\
\text { Dian }\end{array}$ & $\begin{array}{l}\text { If Finned Tubes } \\
\text { Fins/m (Fins/in.) __ Fin Material }\end{array}$ \\
\hline $\begin{array}{l}\text { Tube-to-baffle (in.) } 0.01 \\
\text { Bundle-to-shell, (in.) } 4\end{array}$ & $\begin{array}{l}\text { Diameter, mm (in.), Root }=\text { Over Fins } \\
\text { If Enhanced Surface Tubes }\end{array}$ \\
\hline Number of Baffles Along Length of Shell $\frac{3}{3}$ & (Describe) \\
\hline 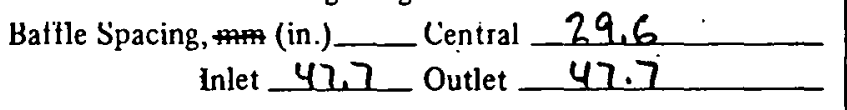 & DETUNING BAFFLE \\
\hline $\begin{array}{l}\text { Unsupported Tube Span Lengths, (in.) } \\
\text { Longest } 77.3 \text { Inlet } 77.3 \text { Outlet } 77.3\end{array}$ & $\begin{array}{l}\text { If Detuning Baffle Used to Control Acoustic . } \\
\text { Vibration, Indicate Position on Sketch Below }\end{array}$ \\
\hline
\end{tabular}

\section{COMMENTS AND SKETCH}

Complete sketches by drawing in tubeside and sheliside nozzles.

Indicate inlet nozzles with on arrow.

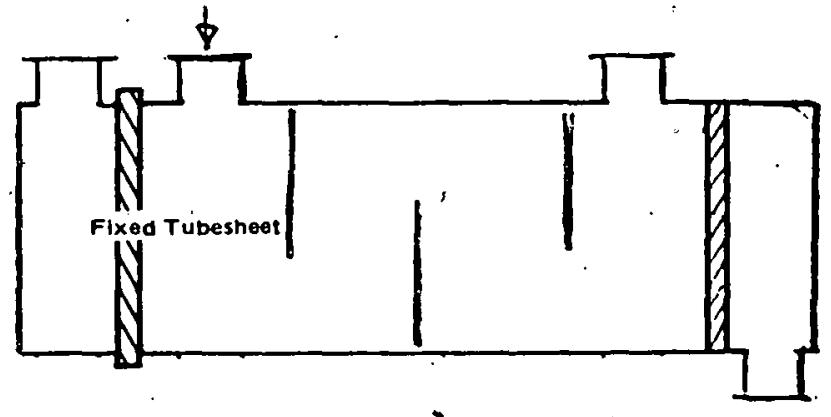

Show shellside Inlet nozzle location, baffle cut oricntation, and impingement devices. .

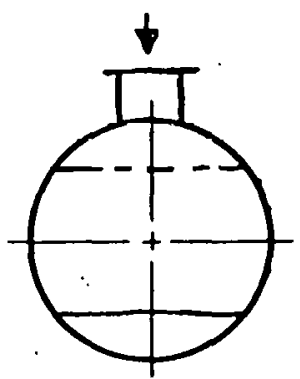




\begin{tabular}{|l|l|}
\hline $\begin{array}{l}\text { Assigned } \\
\text { Case No. } 101\end{array}$ & 1 \\
\hline & $\vdots$
\end{tabular}

PROCESS CONDITIONS

\begin{tabular}{|c|c|c|c|c|}
\hline & \multicolumn{2}{|c|}{ Reference Condition A } & \multicolumn{2}{|c|}{ Reference Condition B } \\
\hline . & Tubeside & Shellside & - Tubeside & Shellside \\
\hline Flow Rate, $\left(10^{3} \mathrm{lb} / \mathrm{hr}\right)$ & $?$ & 184 & & \\
\hline Inlet Temperature, $\in(\mathrm{F})$ & 69.8 & 37.4 & & \\
\hline Outlet Temperature, $\mathrm{e}(\mathrm{F})$ & 53.6 & 54.5 & & \\
\hline Inlet Pressure, $\mathbf{k P}$ (psia) Absolute & 370 & 370 & & \\
\hline Measured $\triangle P, k \mathbb{P}_{Q}(\mathrm{psi}) \quad$ CALCUCATED & 0.34 & 0.9 & & \\
\hline Inlet Weight Fraction Vapor & 1.0 & 1.0 & & \\
\hline Outlet Weight Fraction Vapor & 1.0 & 1.0 & & \\
\hline Vibration Observed & \multicolumn{2}{|l|}{ 口No } & \multicolumn{2}{|c|}{ DYes } \\
\hline
\end{tabular}

\section{FLUID PHYSICAL PROPERTIES}

\begin{tabular}{|c|c|c|c|c|}
\hline Fill In All Applicable Entries & \multicolumn{2}{|c|}{ Tubeside } & \multicolumn{2}{|c|}{ Shellside } \\
\hline Fluid Name & \multicolumn{2}{|c|}{ "CITY GAS" } & \multicolumn{2}{|c|}{ "CITY GAS" } \\
\hline Reference Temperaturc $e(F)$ & 69.8 & 53,6 & 37.4 & 54.5 \\
\hline \multicolumn{5}{|l|}{$\begin{array}{l}\text { Liquid Properties at Reference } \\
\text { Temperatures }\end{array}$} \\
\hline \multicolumn{5}{|l|}{ Density, $\mathrm{kg} / \mathrm{m}^{3}\left(\mathrm{lb} / \mathrm{ft}^{3}\right)$} \\
\hline \multicolumn{5}{|l|}{ Viscosity, $\mathrm{mPa} \cdot \mathrm{s}(\mathrm{cP})$} \\
\hline \multicolumn{5}{|l|}{ Thermal Conductivity, $\mathrm{W} / \mathrm{m} \cdot \mathrm{C}(\mathrm{Btu} / \mathrm{m} \mathrm{ft} \mathrm{F})$} \\
\hline \multicolumn{5}{|l|}{ Heat Capacity, $\mathrm{kJ} / \mathrm{kg} \cdot \mathrm{C}(\mathrm{Btu} / \mathrm{m} \mathrm{ft} \mathrm{F)}$} \\
\hline \multicolumn{5}{|l|}{$\begin{array}{l}\text { Vapor or Gas Properties at } \\
\text { Reference Temperatures }\end{array}$} \\
\hline Density, $\mathrm{k}^{3}\left(\mathrm{lb} / \mathrm{ft}^{3}\right)$ & 0.82 & 0.82 & 0.825 & 0.825 \\
\hline Viscosity, (cP) & $\cdot$ & & 0.0146 & 0.0146 \\
\hline \multicolumn{5}{|l|}{ Thermal Conductivity, $\mathrm{W} / \mathrm{m}-\mathrm{G}(\mathrm{Btu} / \mathrm{hr} \mathrm{ft} \mathrm{F})$} \\
\hline Heat Capacity, kJ/Agof (Btu/lb F) & & & & \\
\hline \multicolumn{5}{|l|}{ - Fluid Molecular Weight, (lb/mole) } \\
\hline \multicolumn{5}{|l|}{ If Boiling or Condensing } \\
\hline Latent Heat, KHAg (Btu/lb) & & & - & \\
\hline
\end{tabular}




\begin{tabular}{|l|l|}
\hline Assigned & $10: 1$ \\
Case No. & 101 \\
\hline
\end{tabular}

VIBRATION AND DAMAGE DESCRIPTION

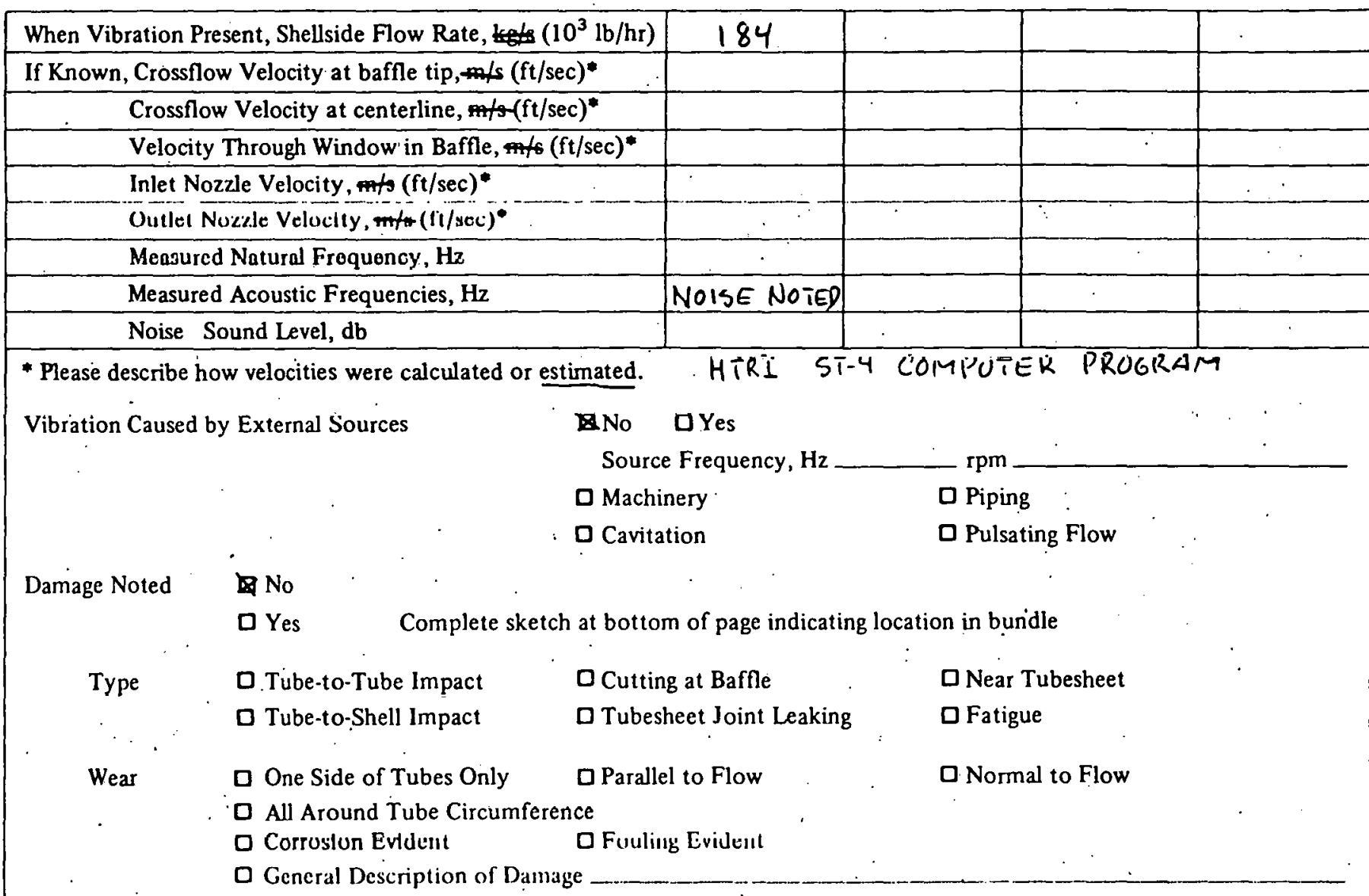

Exchanger Operation History

- How Long on Stream Before Damage Occurred?

- Any Unusual Occurrence Observed Prior to Vibration as a Consequence of
DStart-up
口 Plant-Upset
$\square$ Shutduwn

Describe

- If Vibration Remedy Applied, Describe and Indicate Results:

TUBE BUNDLE DAMAGE SKETCH
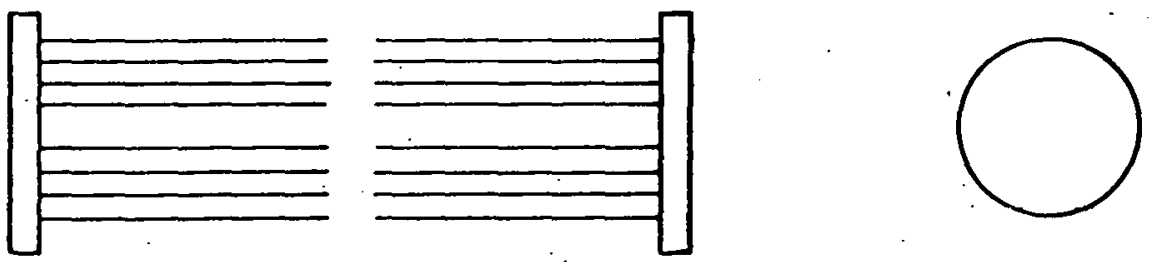

$2-6$ 
To protect the identity of the organization submitting this case, HTRI has assigned a case number. Additionally the data on pages 2, 3, and 4 have been reviewed to ensure that they do not include any propietary information.

Assigned

Case No.

This is a replacement for the original page 1 that provides space for additional comments, drawings, photographs, etc.

Summary

This 44-in. diameter by 12-ft long BEJ TEMA style heat exchanger with segmental baffles had propylene vapor on the shell side and cooling water on the tube side. The vapor entered the shell through the double nozzles. The vapor was cooled but not condensed. Tube damage was noted as cutting at the baffles; however, the specific location in the bundle was not noted. Information is limited to that presented on dața form. 


\begin{tabular}{|l|}
\hline Assigned \\
Case No. 102 \\
\hline
\end{tabular}

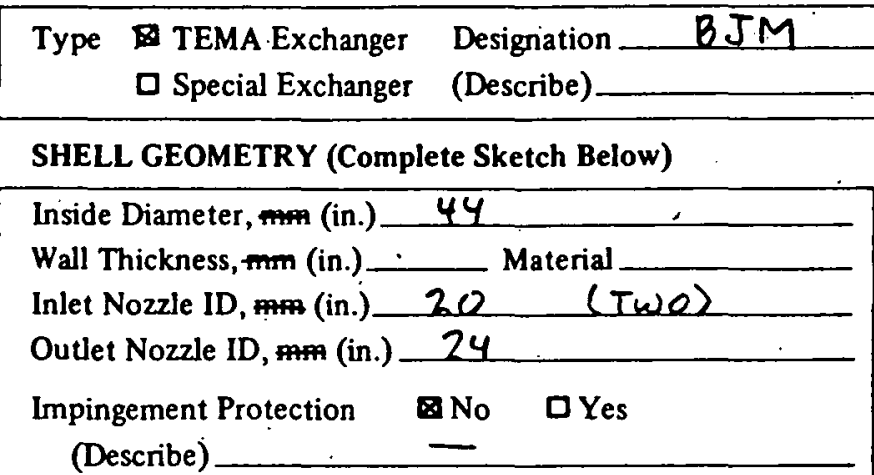

Annular Distributor a $\quad$ No Yes

Open Cut Area ${ }^{2}$ (in. ${ }^{2}$ )

Nozzle-to-First Tube Row Distance, (in.)

\section{CROSS BAFFLE GEOMETRY}

Type Segmental; $\square$ Double-Segmental $\square$ Disc/Doughnut

$\square$ Triple-Segmental; $\square$ No-Tubes-in-Window

Baffle Cut, \% Shell Diameter 46

Cut Orientation Relative to Axis of Inlet Nozzle

Inlet Baffle $\square$ Perpendicular $\otimes$ Parallel $045^{\circ}$

Central Baffles $\square$ Perpendicular EParallel $\square 45^{\circ}$

Baffle Thickness, (in.) 0.625 Material

Diametral Clearances Shell-to-baffe, (in.) 0.225

Tube-to-baffle (in.) 0.015625

Bundle-to-shcll, (in.) 0.50

Number of Baffles Along Length of Shell

Baffle Spacing, (in.) Central 26.375

Inlet 29.75 Outlet 25.375

Unsupported Tube Span Lengths, (in.)

Longest 56.75 Inlet 56.75 Outlet 52.375

\section{COMMENTS AND SKETCH}

Complete sketches by drawing in tubeside and sheliside nozzles.

Indicate intet nozzles with an arrow.

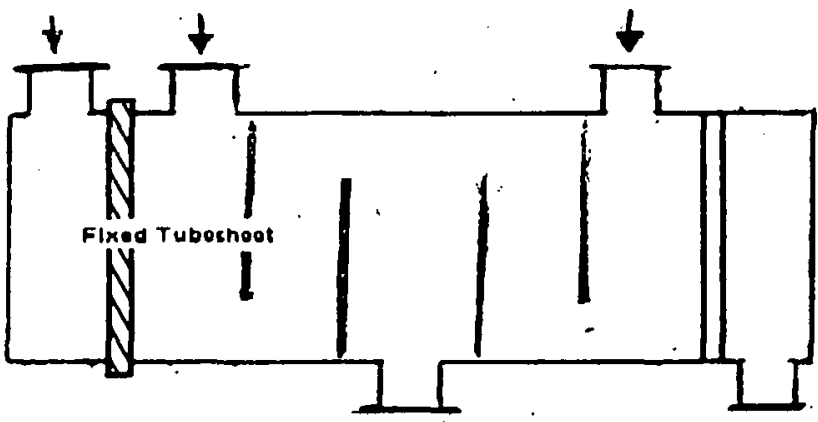

TUBE GEOMETRY

Outside Diameter, (in.) 0i750

Wall Thickness, (in.) 0.065 Material ADMIRACTY

Tube Lengths

BRASS

Straight Tube, Inside Tubesheets, (in.) 140.25

U-Tube, Tubesheet to Bend Tangent, $\mathrm{mm}$ (in.)

Tube Pitch, (in.) 0.9375

Layout (Please Circle)

$$
\text { Flow } 30^{\circ} \text { ofo }
$$

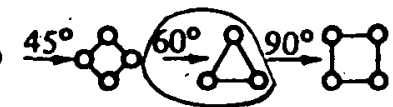

Nọ. uf Tules INo, of Tubepasses

First Tubepass $\otimes$ Countercurrent $\otimes$ Cocurrent

Tubc-to-Tubesheet Joint

$\square$ Welded $\mathbf{Q}$ Roller Expanded $\mathbf{D}$ Other

If U-Tube

Maximum Bend Radius, mm.(in.)

Bend Orientation Relative to Axis of Shellside Inlet Nozzle

口 Perpendicular Q Parallel

If Bend Supported, Describe in Comments Below

If Finned Tubes

Fins/m (Fins/in.) _ _ Fin Material _ -

Diameter, mm (in.), Root - Over Fins -

If Enhanced Surface Tubes

(Describe)

\section{DETUNING BÁFFLE}

If Detuning Baffle Used to Control Acoustic

Vibration, Indicate Position on Sketch Below 


\section{PROC.FSS CONDITIONS}

\begin{tabular}{|c|c|c|c|c|}
\hline \multirow{2}{*}{$\cdot$} & \multicolumn{2}{|c|}{ Reference Condition A } & \multicolumn{2}{|c|}{ Reference Condition B } \\
\hline & Tubeside & Shellside & Tubeside & Shellside \\
\hline Flow Rate, $\operatorname{kg}\left(10^{3} \mathrm{lb} / \mathrm{hr}\right)$ & $?$ & 676 & & \\
\hline Inlet Temperature, $\mathrm{e}(\mathrm{F})$ & 75 & 182 & & \\
\hline Outlet Temperature, e(F) & 120 & 100 & & \\
\hline Inlet Pressure, $1 \mathbb{P}^{2}$ (psia) Absolute & 69.7 & 236 & & \\
\hline Measured $\Delta P, k P_{\text {(psi) }}$ & 12.4 & 4.0 & & \\
\hline Inlet Weight Fraction Vapor & 0 & 1.0 & & \\
\hline Outlet Weight Fraction Vapor & 0 & 1.0 & & \\
\hline Vibration Observed & DNo & & 口No & \\
\hline
\end{tabular}

\section{FLUID PHYSICAL PROPERTIES}

\begin{tabular}{|c|c|c|c|}
\hline \multirow{3}{*}{$\begin{array}{l}\text { Fill In All Applicable Entries } \\
\text { Fluid Name } \\
\text { Reference Temperature, (F) }\end{array}$} & Tubeside & \multirow{2}{*}{\multicolumn{2}{|c|}{$\begin{array}{r}\text { Shellside. } \\
\text { PROPYLENE }\end{array}$}} \\
\hline & COOLING WATER & & \\
\hline & \begin{tabular}{l|l} 
& \\
\end{tabular} & 100 & 182 \\
\hline \multicolumn{4}{|l|}{ Liquid Properties at Reference } \\
\hline \multicolumn{4}{|l|}{ Temperatures } \\
\hline \multicolumn{4}{|l|}{ Density, $\mathrm{kg}_{\mathrm{g}} / \mathrm{m}^{3}\left(\mathrm{lb} / \mathrm{ft}^{3}\right)$} \\
\hline \multicolumn{4}{|l|}{ Viscosity, mPa $(\mathrm{cP})$} \\
\hline \multicolumn{4}{|l|}{ Thermal Conductivity, $/ \mathrm{m} \cdot \epsilon(\mathrm{Btu} / \mathrm{m} \mathrm{ft} \mathrm{F})$} \\
\hline \multicolumn{4}{|l|}{ Heat Capacity; $\mathbf{k} \mathbf{H} / \mathbf{k g} \cdot \in(B t u / m$ ft F) } \\
\hline \multicolumn{4}{|l|}{$\begin{array}{l}\text { Vapor or Gas Properties at } \\
\text { Reference Temperatures }\end{array}$} \\
\hline Density, $\mathrm{m}^{3}\left(\mathrm{lb} / \mathrm{ft}^{3}\right)$ & & 1.62 & 1.44 \\
\hline$V_{\text {iscosity, rmPa-s (cP) }}$ & & 0.0087 & 0.0095 \\
\hline \multicolumn{4}{|l|}{ Thermal Conductivity, $=\mathrm{W} / \mathrm{m}-\mathrm{C}(\mathrm{Btu} / \mathrm{hr} \mathrm{ft} \mathrm{F})$} \\
\hline \multicolumn{4}{|l|}{ Heat Capacity, kf/kg $\in$ (Btu/lb F) } \\
\hline \multicolumn{4}{|l|}{ Fluid Molecular Weight, kgtmot (lb/mole) } \\
\hline \multicolumn{4}{|l|}{ If Boiling or Condensing } \\
\hline Latent Heat, $\mathrm{kJ} / \mathrm{kg}(\mathrm{Btu} / \mathrm{lb})$ & - & & \\
\hline
\end{tabular}




\begin{tabular}{l} 
Assigned \\
Case No. 102 \\
\hline
\end{tabular}

\section{VIBRATION AND DAMAGE DESCRIPTION}

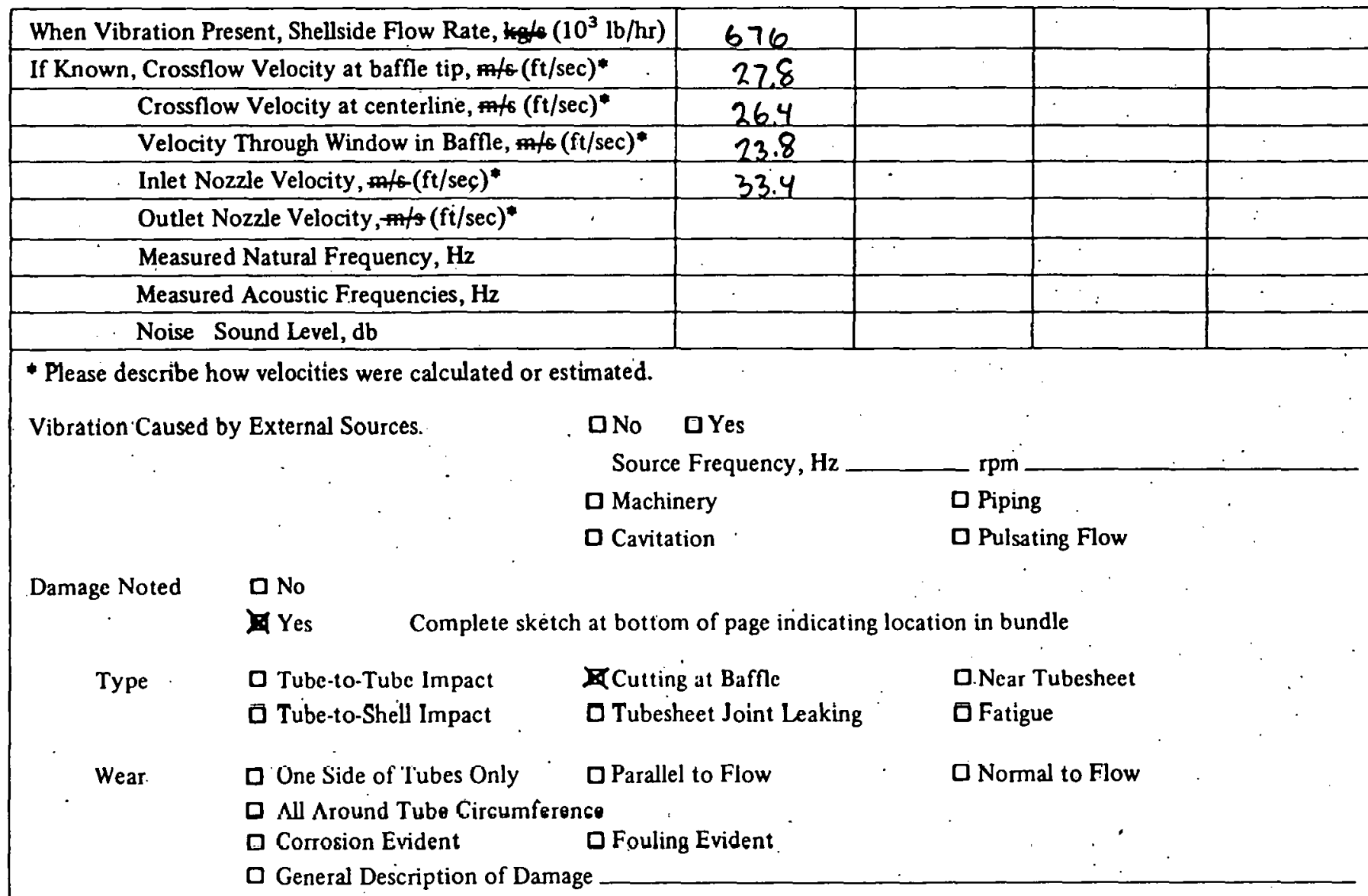

Exchanger Operation History

- How Long on Stream Before Damage Occurred?

- Any Unusual Occurrence Observed Prior to Vibration as a Consequence of QStart-up 口 Plant-Upset $\square$ Shutdown

Describe

- If Vibration Remedy Applied, Describe and Indicate Results:

TUDE DUNDLE DAMAGE SKETCII
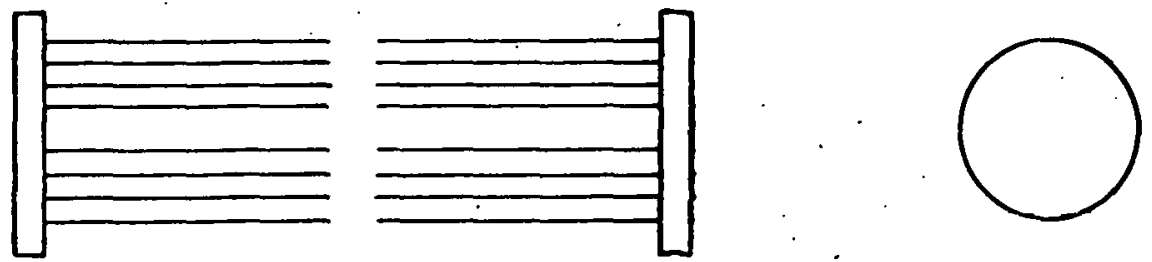

$2-10$ 
To protect the identity of the organization submitting this case, HTRI has assigned a case number. Additionally the data on pages 2,3 , and 4 have been reviewed to ensure that they do not include any propietary information.

This is a replacement for the original page 1 that provides space for additional comments, drawings, photographs, etc.

\section{Summary}

This 20-in. diameter by 7-ft long AEP TEMA style heat exchanger with segmental baffles had water on both the shell side and the tube side. The inlet nozzle orientation was such that its centerline intersected the baffle cut at $45^{\circ}$. The baffle cut was 45 percent of the shell inside diameter which results. in little crossflow. The tube damage reported tube-to-tube impact resulting in splitting of the tube material until leaks developed. Information is limited to that presented on data form. 


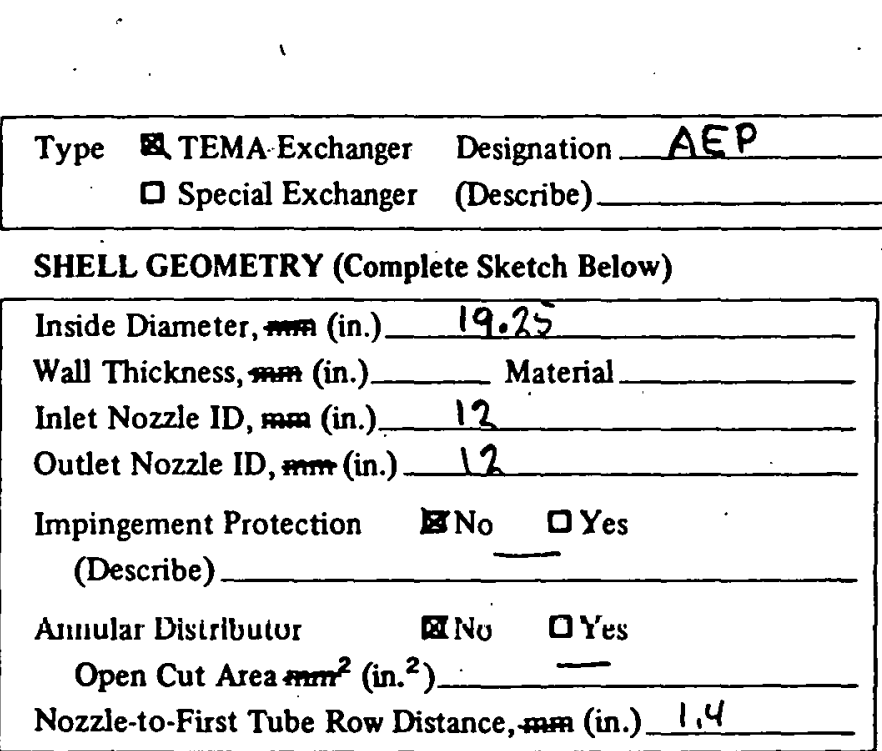

\section{CROSS BAFFLE GEOMETRY}

Type Regmental; $\square$ Double-Segmental $\square$ Disc/Doughnut 口 Triple-Segmental; $\square$ No-Tubes-in-Window

Baffle Cut, \% Shell Diameter 45

Cut Orientation Relative to Axis of Inlet Nozzle

Inlet Baffle $\square$ Perpendicular $\square$ Parallel $45^{\circ}$

Central Baffles $\square$ Perpendicular $\square$ Parallel $45^{\circ}$

Baffle Thickness, (in.) 0.375 Material

Diametral Clearances Shell-to-baffle, (in.) 0.141

Tube-to-baffle (in.) $\quad 0.019$

Bundle-to-shell, (in.) 2.812

Number of Baffles Along Length of Shell __ 3

Battle Spacing, (in.) Central 21

$$
\text { - Inlet } 21 \text { Outlet } 21
$$

Unsupported Tube Span Lengths, (in.)

Longest 42 inlet Outlet

\begin{tabular}{l} 
Assigned \\
Case No. 103 \\
\hline
\end{tabular}

Shell Orientation Horizontal

DVertical

\section{TUBE GEOMETRY}

Outside Diameter, (in.) $\quad 0.625$

Wall Thickness, (in.) 0.049 Material $90 / 10 \mathrm{CuNi}$

Tube Lengths

Straight Tube, Inside Tubesheets, (in.) 84

U-Tube, Tubesheet to Bend Tangent, (in.) -

Tube Pitch, min (in.) $\quad 0.750$

Layout (Please Rirrle)

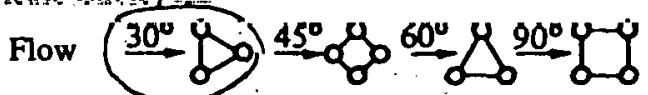

No. of Tubes 407

No. of Tubepasses

First Tubepass $\square$ Countercurrent $\square$ Cocurrent

Tube-tu-Tubesheet Joint

a Welded Roller Expanded 0 Other

If U-Tube

Maximum Bend Radius, mm (in.)

Bend Orientation Relative to Axis of Shellside Inlet Nozzle

$\square$ Perpendicular $\square$ Parallel

If Bend Supported, Describe in Comments Below

If Finned Tubes

Fins/m (Fins/in.) _ _ Fin Material _ -

Diameter, mm (in.), Root - Over Fins _ -

If Enhanced Surface Tubes

(Describe)

\section{DETUNING BAFFLE}

If Detuning Baffle Used to Control Acoustic

Vibration, Indicate Position on Sketch Below

\section{COMMENTS AND SKETCH}

Complete sketches by drawing in tubeside and shellside nozzles.

Indicate Inlet nozples with an arrow.

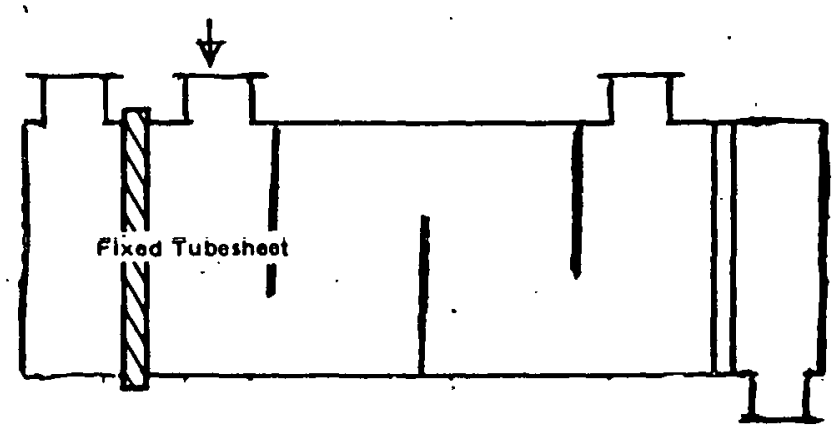

Show shellslde Inlot nozzle location, batfle cut orientation, and impingement devices.

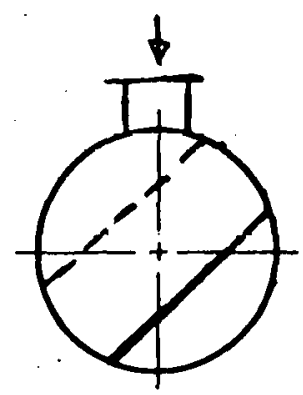

PAIR OF SEAL STRIPS. $\leadsto$ indicate top of exchanger as mounted 


\begin{tabular}{|c|}
\hline $\begin{array}{l}\text { Assigned } \\
\text { Case No. } 103\end{array}$ \\
\hline
\end{tabular}

\section{PROCESS CONDITIONS}

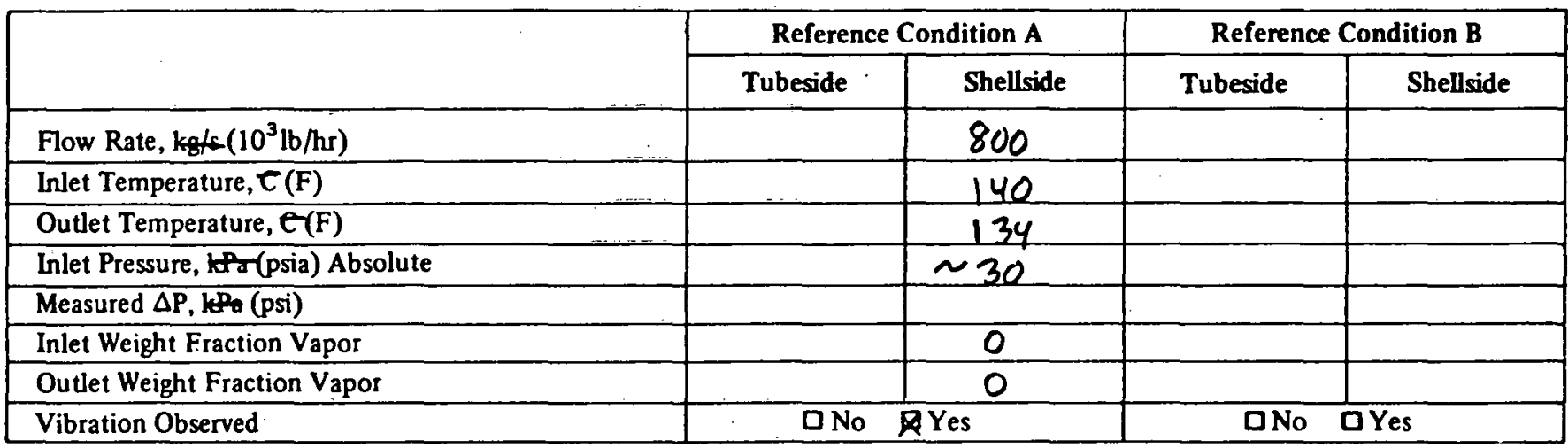

\section{FLUID PHYSICAL PROPER TIES}

\begin{tabular}{|c|c|c|}
\hline Fill In All Applicable Entries & Tubeside & Shellside \\
\hline Fluid Name & WATER & WATER \\
\hline Reference Temperature, C (F) & & \\
\hline \multicolumn{3}{|l|}{$\begin{array}{l}\text { Liquid Properties at Reference } \\
\text { Temperatures }\end{array}$} \\
\hline Density, $\mathrm{kg} / \mathrm{m}^{3}\left(\mathrm{lb} / \mathrm{ft}^{3}\right)$ & & \\
\hline Viscosity, $\mathrm{mPa} \cdot \mathrm{s}(\mathrm{cP})$ & & \\
\hline Thermal Conductivity, $\mathrm{W} / \mathrm{m} \cdot \mathrm{C}(\mathrm{Btu} / \mathrm{m} \mathrm{ft} \mathrm{F})$ & & \\
\hline Heat Capacity, $\mathrm{kJ} / \mathrm{kg} \cdot \mathrm{C}(\mathrm{Btu} / \mathrm{m} \mathrm{ft} \mathrm{F)}$ & & \\
\hline $\begin{array}{l}\text { Vapor or Gas Properties at } \\
\text { Reference Temperatures }\end{array}$ & & \\
\hline Density, $\mathrm{kg} / \mathrm{m}^{3}\left(\mathrm{lb} / \mathrm{ft}^{3}\right)$ & & \\
\hline Viscosity, mPa.s (cP) & & \\
\hline Thermal Conductivity, $\mathrm{W} / \mathrm{m} \cdot \mathrm{C}(\mathrm{Btu} / \mathrm{hr} \mathrm{ft} \mathrm{F})$ & & \\
\hline Heat Capacity, $\mathrm{kJ} / \mathrm{kg} \cdot \mathrm{C}(\mathrm{Btu} / \mathrm{lb} \mathrm{F})$ & & \\
\hline Fluid Molecular Weight, $\mathrm{kg} / \mathrm{mol}(\mathrm{b} / \mathrm{mole})$ & & \\
\hline If Boiling or Condensing & & \\
\hline Latent Heat, $\mathrm{kJ} / \mathrm{kg}(\mathrm{Btu} / \mathrm{lb})$ & & \\
\hline
\end{tabular}




\begin{tabular}{l}
\hline Assigned, 103 \\
Case No. 103 \\
\hline
\end{tabular}

VIBRATION AND DAMAGE DESCRIPTION

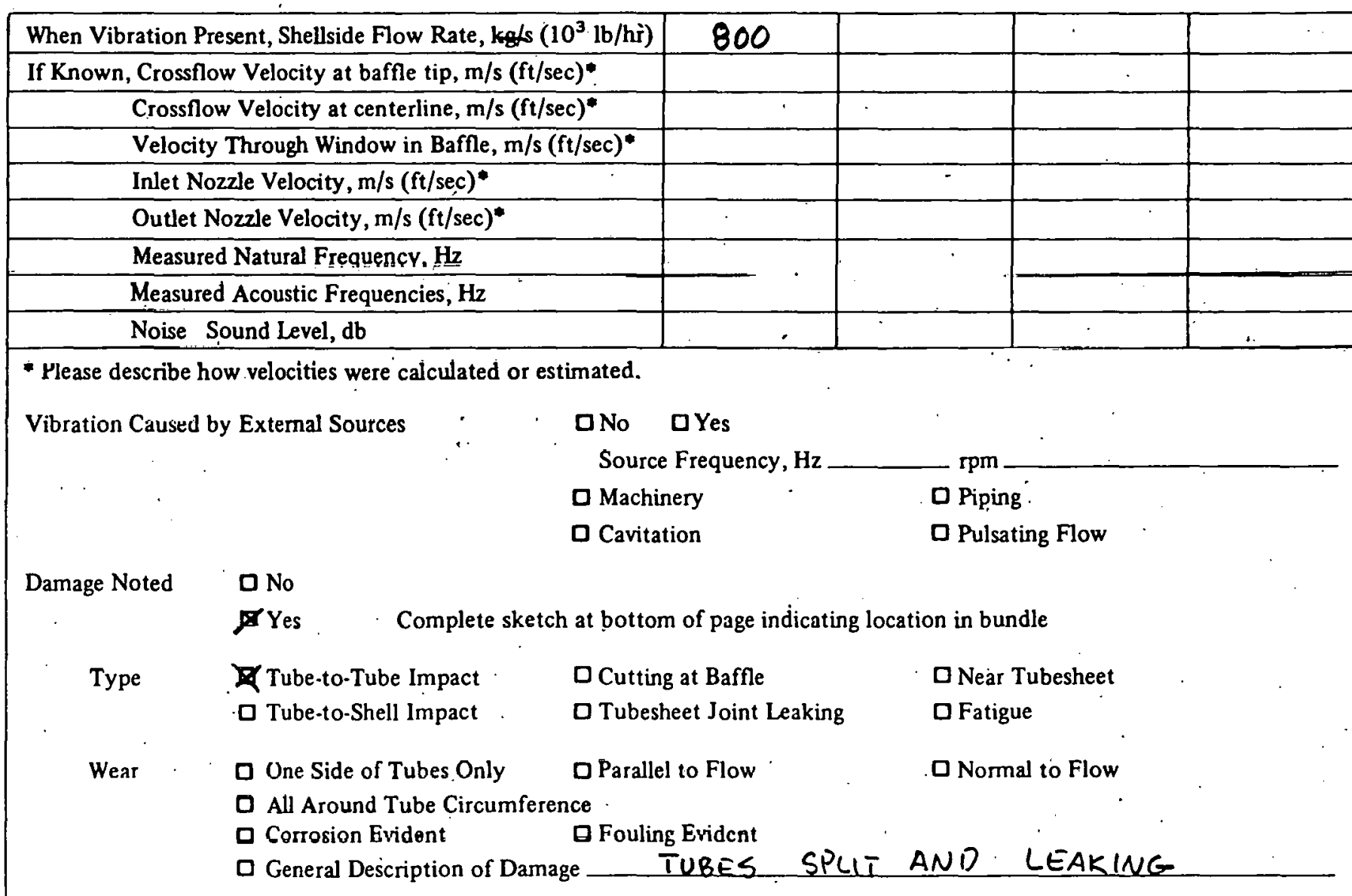

Exchanger Operation History

- How Long on Stream Before Damage Occurred?

- Any Unusual Occurrencc Obscrved Prior to Vibration as a Consequence of

DStart-up Q D Plant-Upset Dhutdown

Describe

- If Vibration Remedy Applied, Describe and Indicate Results:

TUBE BUNDLE DAMAGE SKETCII
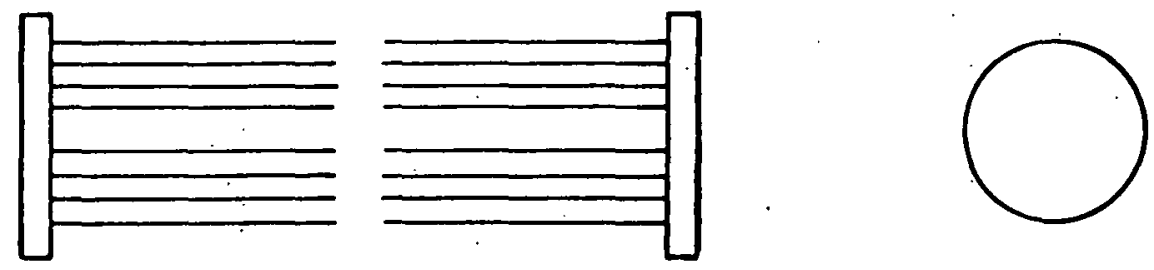

$2-14$ 
To protect the identity of the organization submitting this case, HTRI has assigned a case number. Additionally the data on pages 2,3 , and 4 have been reviewed to ensure that they do not include any propietary information.

This is a replacement for the original page 1 that provides space for additional comments, drawings, photographs, etc.

\section{Summary}

This 10-in. diameter by 6-ft long AES TEMA style heat exchanger with segmental baffles had air on the shell side and water on the tube side. The inlet nozzle orientation was such that its centerline intersected the baffle cut at $45^{\circ}$. The baffle cut was 45 percent of the shell inside diameter which results in little crossflow. All tubes showed evidence of cutting at each baffle. It was noted that there was a possibility that the vibration might have been induced by outside sources. Information is limited to that presented on data form. 


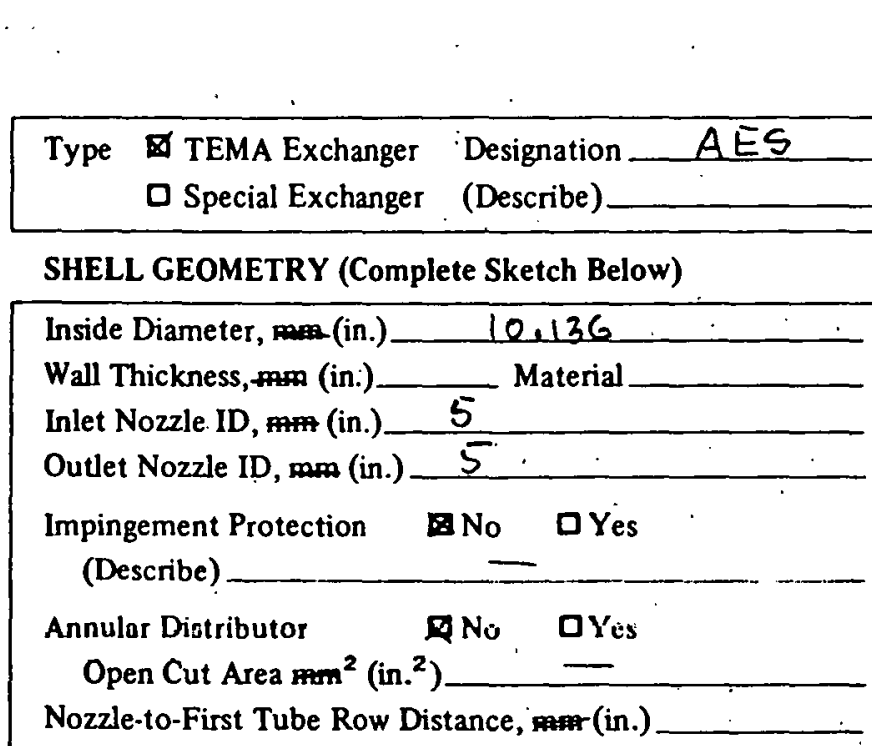

\section{CROSS BAFFLE GEOMETRY}

Type Segmental; $\square$ Double-Segmental $\square$ Disc/Doughnut

口 Triple-Segmental; $\square$ No-Tubes-in-Window

Baffle Cut, \% Shell Diameter 45

Cut Orientation Relative to Axis of Inlet Nozzle

Inlet Baffle $\square$ Perpendicular $\triangle$ Parallel $45^{\circ}$

Central Baffles $\square$ Perpendicular $\square$ Parallel $45^{\circ}$

Baffle Thickness, (in.) 0.375 Material

Diametral Clearances Shell-to-baffle, (in.) 0.090

Tube-to-baffle (in.) 0.019

Bundle-to-shell, (in 2.636

Number of Baffles Along length of Shell $\quad 6$

$D_{a} f$ th 3yatints,

Inlet 10 Outlet 10

Unsupported Tube Span Lengths, (in.)

Longest 20 Inlet 20 Outlet 20

\section{TUBE GEOMETRY}

Outside Diameter, m (in.) $\quad 0.625$

Wall Thickness, mm (in.) 0.065 Material $90 / 10 \mathrm{CuNi}$

Tube lengths

Straight Tube, Inside Tubesheets, (in.) 70

U-Tube, Tubesheet to Bend Tangent, (in.) _-

Tube Pitch, am (in.) _ 0.750

Layout (Please Circle)

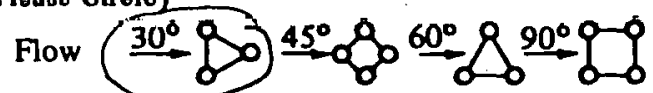

No. of Tubes 78 No. of Tubepasses _ _

First Tubepass $\square$ Countercurrent $\square$ Cocurrent

Tube-to-Tubesheet Joint

$\square$ Welded $\square$ Roller Expanded DOther NOT GIUEN

If U-Tube

Maximum Bend Radius, mm (in.)

Bend Orientation Relative to Axis of Shellside Inlet Nozzle

D Perpendicular $\square$ Parallel

If Bend Supported, Describe in Comments Below

If Finned Tubes

Fins/m (Fins/in.) _ _ _ Fin Material __ _

Diameter, mm (in.), Root - Over Fins _ -

If Enhance.d Surface Tubes

(Describe)

\section{DETUNING BAFFLE}

If Detuning Baffle Used to Control Acoustic

Vibration, Indicate Position on Sketch Below

\section{COMMENTS AND SKETCH}

Complete sketches by drawing In tubeslde and shellside nozzles.

Indicate Inlet nozzles with an arrow.

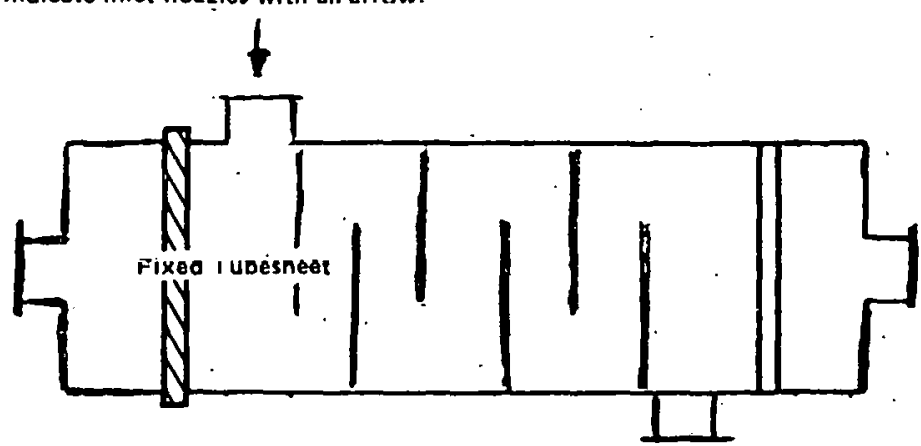

$\hookrightarrow$ indicate top of exchanger as mounted by fllling in appropriate arrow.
Show sheliside Inlet nozzle location, baffle cut orlentation, and impingement devices.

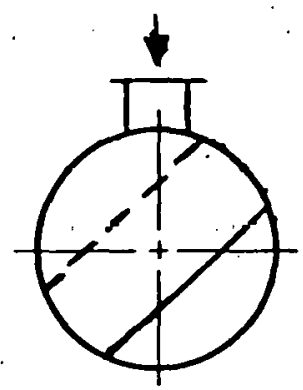

Two PaIRS. OF SEAL STRIPS 
PROCESS CONDITIONS

\begin{tabular}{|c|c|c|c|c|c|}
\hline & & \multicolumn{2}{|c|}{ Reference Condition A } & \multicolumn{2}{|c|}{ Reference Condition B } \\
\hline Flow Rate, $\left(10^{3} \mathrm{lb} / \mathrm{hr}\right)$ & & & 10.476 & & \\
\hline Inlet Temperature, $\in(F)$ & & 163.7 & 530 & & \\
\hline Outlet Temperature, $\mathrm{E}(\mathrm{F})$ & & 170.6 & 350 & . & \\
\hline Inlet Pressure, 19a (psia) Absolute & . & & 113 & & ? \\
\hline Inlet Weight Fraction Vapor & & & 1.0 & & \\
\hline Outlet Weight Fraction Vapor & & & 1.0 & & . \\
\hline Vibration Observed & & \multicolumn{2}{|c|}{ DNo $\mathbf{X Y e s}$} & \multicolumn{2}{|c|}{ DYes } \\
\hline
\end{tabular}

FLUID PHYSICAL PROPERTIES

\begin{tabular}{|c|c|c|c|}
\hline Fill In All Applicable Entries & Tubeside & Shellside & \\
\hline \multicolumn{4}{|l|}{ Reference Temperature C (F) } \\
\hline Liquid Properties at Reference & & & \\
\hline Temperatures & & & \\
\hline Viscosity, $\mathrm{mPa} \cdot \mathrm{s}(\mathrm{cP})$ & & & \\
\hline \multicolumn{4}{|l|}{ Thermal Conductivity, $\mathrm{W} / \mathrm{m} \cdot \mathrm{C}(\mathrm{Btu} / \mathrm{m} \mathrm{ft} \mathrm{F})$} \\
\hline \multicolumn{4}{|l|}{ Heat Capacity, $\mathrm{kJ} / \mathrm{kg} \cdot \mathrm{C}(\mathrm{Btu} / \mathrm{m}$ ft F $)$} \\
\hline \multicolumn{4}{|l|}{ Reference Temperatures } \\
\hline \multicolumn{4}{|l|}{ Density, $\mathrm{kg} / \mathrm{m}^{3}\left(\mathrm{lb} / \mathrm{ft}^{3}\right)$} \\
\hline \multicolumn{4}{|l|}{ Thermal Conductivity, $\mathrm{W} / \mathrm{m} \cdot \mathrm{C}(\mathrm{Btu} / \mathrm{hr} \mathrm{ft} \mathrm{F})$} \\
\hline \multicolumn{4}{|l|}{ Heat Capacity, $\mathrm{kJ} / \mathrm{kg} \cdot \mathrm{C}(\mathrm{Btu} / \mathrm{lb} \mathrm{F})$} \\
\hline \multicolumn{4}{|l|}{ Fluid Molecular Weight, $\mathrm{kg} / \mathrm{mol}(\mathrm{lb} / \mathrm{mole})$} \\
\hline \multicolumn{4}{|l|}{ if Boiling or Condensing } \\
\hline Latent Heat, $\mathrm{kJ} / \mathrm{kg}(\mathrm{Btu} / \mathrm{lb})$ & & & \\
\hline
\end{tabular}




\begin{tabular}{|l|}
\hline Assigned . \\
Case No. $\quad 104$ \\
\hline
\end{tabular}

\section{VIBRATION AND DAMAGE DESCRIPTION}

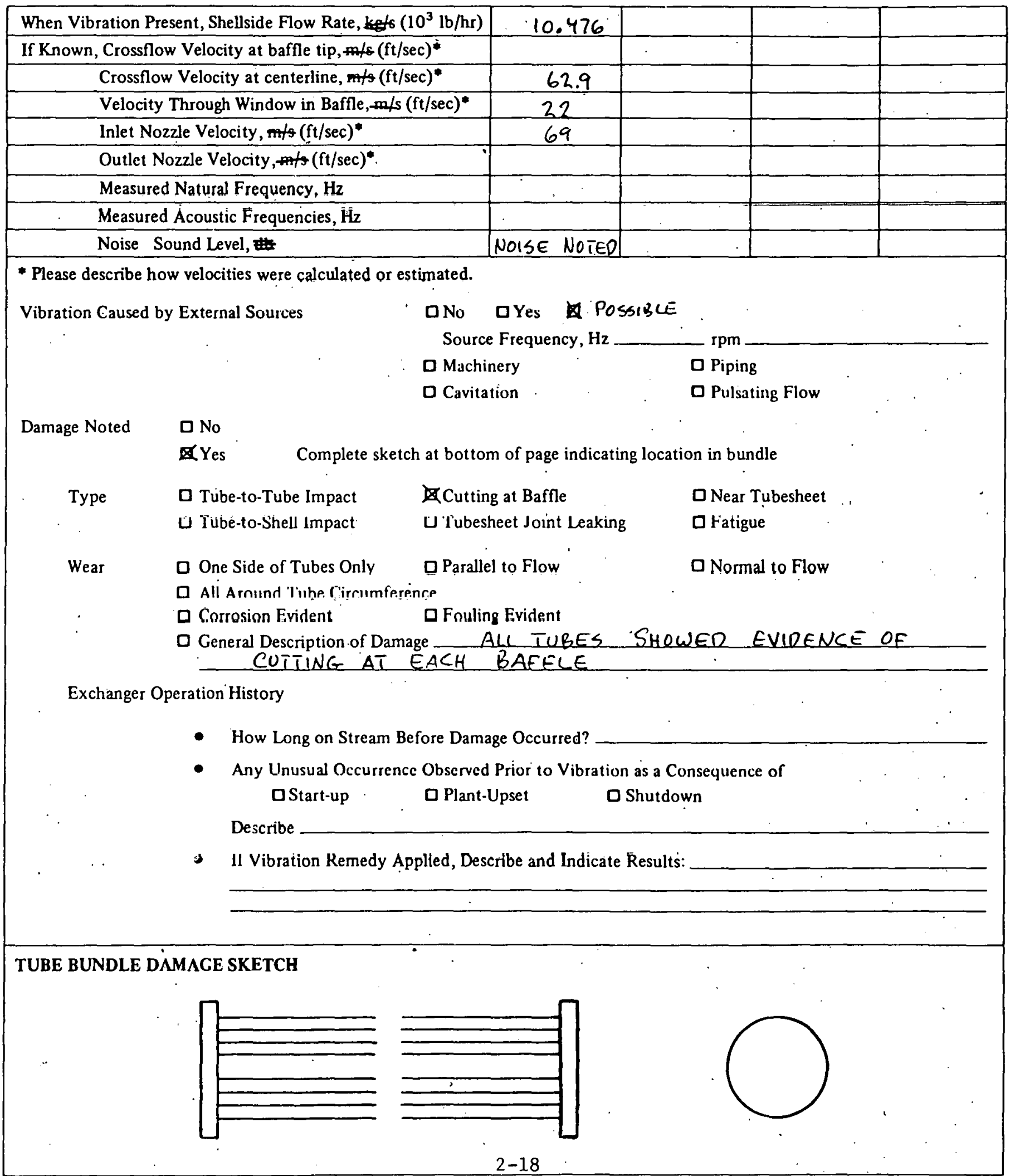


To protect the identity of the organization submitting this case, HTRI has assigned a case number. Additionally the data on pages 2, 3, and 4 have been reviewed to ensure that they do not include any propietary information.

This is a replacement for the original page 1 that provides space for Assigned Case No. additional comments, drawings, photographs, etc.

Summary

This 57-in. diameter by 12-ft long AET TEMA style heat exchanger with segmental baffles had acetylene on the shellside and water on the tubeside. The tube span for baffle window tubes at the inlet and the outlet greatly exceeded the TEMA standards for maximum spans for $1-i n$. carbon steel tubes. However, for this case no vibration was noted for the given flow rates. Information is limited to that presented on data form. 


\begin{tabular}{|l|}
\hline Assigned \\
Case No. 105 \\
\hline
\end{tabular}

Type $\begin{array}{ll}\text { \& TEMA Exchanger } & \begin{array}{l}\text { Designation } \\ \text { (Describe) }\end{array}\end{array}$

SHELL GEOMETRY (Complete Sketch Below)

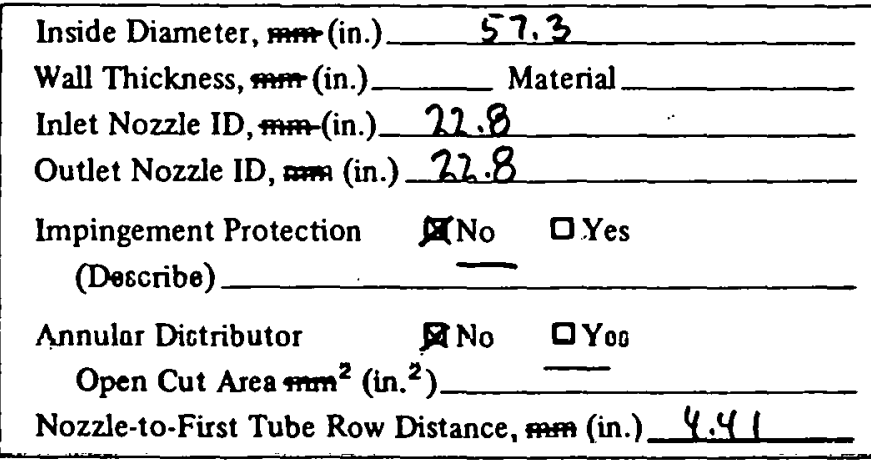

\section{CROSS BAFFLE GEOMEIRY}

\section{Type Segmental; $\square$ Double-Segmental $\square$ Disc/Doughnut}

D Triple-Segmental; D No-Tubes-in-Window

Baffle Cut, \% Shell Diameter $\frac{44}{\text { Cut Orientation Relative to Axis of Inlet Nozzle }}$

Inlet Baffle $\square$ Perpendicular $\mathbb{Q}$ Parallel $\square 45^{\circ}$

Central Baffles $\square$ Perpendicular Parallel $\square 45^{\circ}$

Baffle Thickness, (in.) 0.625 Material

Diametral Clearances Shell-to-baffle, (in.) 0.27

$$
\text { Tube-to-baffle mm (in.) } 0.02
$$

Bundle-to-shell, (in.) 3.74

Number of Baffles Along Length of Shell __ 6

Baffle Spacing, (iin.) $=$ Central 34.65 Inlet 55.5 Outlet 40.56

Unsupported Tube Span Lengths, (in.)

Longest 90.15 Inlet 90.15 outlet 75.21
Shell Orientation

\&Horizontal Dertical

\section{TUBE GEOMETRY}

Outside Diameter, (in.) $\quad 0.988$

Wall Thickness, (in.) 0.079 Material CS

Tube Lengths

Straight Tube, Inside Tubesheets, (in.) 234.65

U-Tube, Tubesheet to Bend Tangent, (in.) -

Tube Pitch, $\operatorname{mm}($ in.) $\quad 1.26$

Layout (Please Circle)

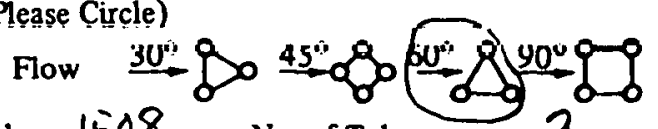

No. of Tubes 1508

INo. of Tubepasses 2

First Tubepass $\otimes$ Countercurrent 0 Cocurrent

Tube-to-Tubesheet Joint

RWelded $\square$ Roller Expanded $\square$ Other

If U-Tube

Maximum Bend Radius, ma (in.)

Bend Orientation Relative to Axis of Shellside Inlet Nozzle

D Perpendicular D Parallel

If Bend Supported, Describe in Comments Below

If Finned Tubes

Fing/m (Fins/in.) _ _ Fin Material _-

Diameter, mm (in.), Root = Over Fins -

If Enhanced Surface Tubes

(Describe)

\section{DETUNING BAFFLE}

If Deturing Baffle Uséd to Control Acoustic

Vibration, Indicate Position on Sketch Below

\section{COMMENTS AND SKETCH}

Complete sketches by drawing in tubeside and shellslde nozzles.

indicate inlet nozzles with an arrow.

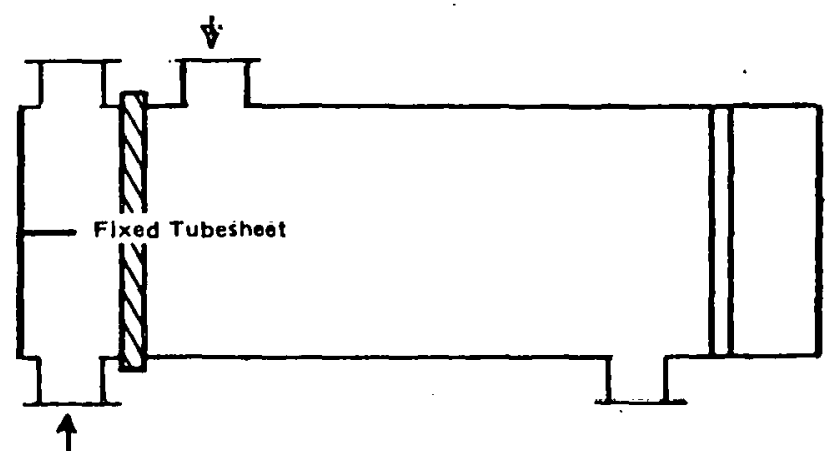

Show shellslde inlet nozzle location, batfle cut orientation, and Impingement devices.

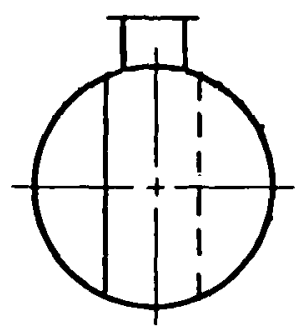




\section{DOE/AŇL/HTRI HEAT EXCHANGER TUBE VIBRATION DATA FORM}

Page 3

\begin{tabular}{l}
\hline Assigned \\
Case No. 105 \\
\hline
\end{tabular}

\section{PROCESS CONDITIONS}

\begin{tabular}{|c|c|c|c|c|c|}
\hline & & \multicolumn{2}{|c|}{ Reference Condition $\mathbf{A}$} & \multicolumn{2}{|c|}{ Reference Condition B } \\
\hline . & & Tubeside & Shellside & Tubeside & Shellside \\
\hline Flow Rate, $\mathrm{kg} / \mathrm{f}\left(10^{3} \mathrm{lb} / \mathrm{hr}\right)$ & & & 351 & & \\
\hline Inlet Temperature, $G(F)$ & & 74.7 & 201 & & \\
\hline Outlet Temperature, $G(F)$ & & 10,4 & 86 & & \\
\hline Inlet Pressure, kP\& (psia) Absolute & $i$ & 654 & 277 & & \\
\hline \multicolumn{6}{|l|}{ Measured $\Delta P, \ldots P_{Q}$-(psi) } \\
\hline Inlet Weight Fraction Vapor & & 0 & 1.0 & & \\
\hline Outlet Weight Fraction Vapor & & 0 & 1.0 & & \\
\hline Vibration Observed & & \multicolumn{2}{|c|}{ GNo DYes } & \multicolumn{2}{|c|}{ DYes } \\
\hline
\end{tabular}

\section{FLUID PHYSICAL PROPERTIES}

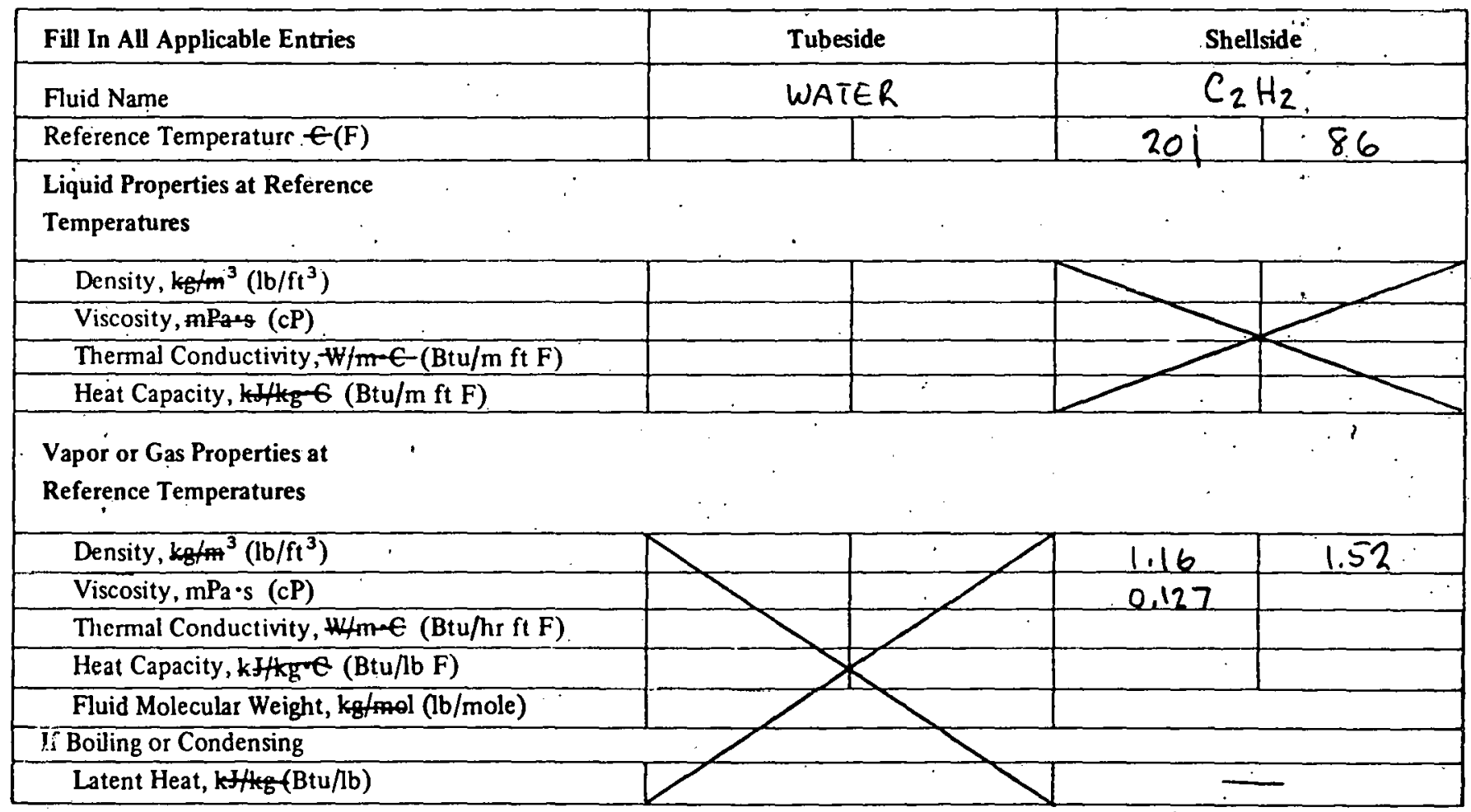




\begin{tabular}{l} 
Assigned \\
Case No. $\quad 105$ \\
\hline
\end{tabular}

VIBRATION AND DAMAGE DESCRIPTION

NO VIBRATION NOTED

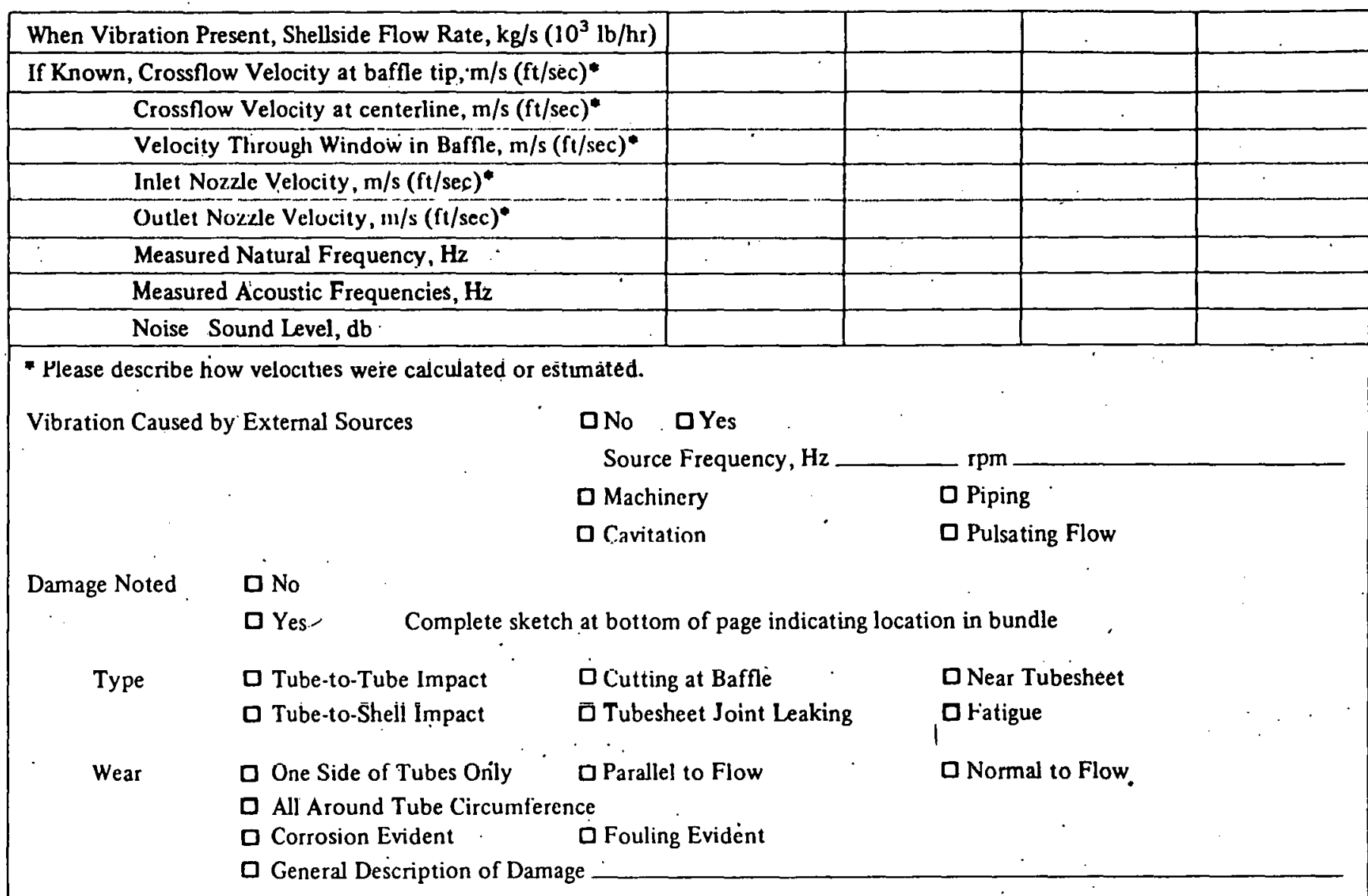

Exchanger Operation History

- How Long on Stream Before Damage Occurred?

- Any Unusual Occurrence Observed Prior to Vibration as a Consequence of

QStart-up Q Plant-Upset Q Shutdown

Describe

- If Vibration Remedy Applied, Describe and Indicate Results:

TUBE BUNDLE DAMAGE SKETCH
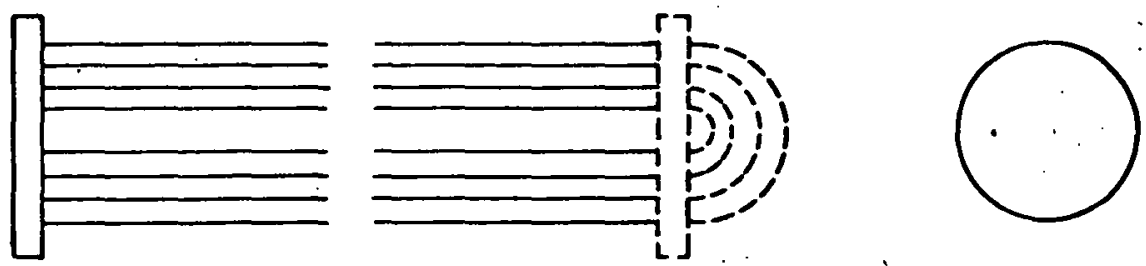

$2-22$ 
To protect the identity of the organization submitting this case, HTRI has assigned a case number. Additionally the data on pages 2,3, and 4 have been reviewed to ensure that they do not include any propietary information.

This is a replacement for the original page 1 that provides space for Assigned Case No. additional comments, drawings, photographs, etc.

\section{Summary}

This 57-in. diameter by 12-ft long AET TEMA style heat exchanger with segmental baffles had acetylene on the shellside and water on the tubeside. The tubespan for the baffle window tubes at the inlet and outlet were nearly the limit specified in the TEMA Standards for $1-i n$. carbon'steel tubes. Flow-induced tube vibration is indicated as the cause for tube damage near the tubesheet. Information is limited to that presented on the data form. 


\begin{tabular}{l} 
Assigned \\
Case No. 106 \\
\hline
\end{tabular}

\begin{tabular}{|c|c|c|}
\hline Type TEMA Exchanger & $\begin{array}{l}\text { Designation_AET } \\
\text { a Special Exchanger }\end{array}$ (Describe) & Shell Orientation $\begin{array}{l}\text { Horizontal } \\
\text { qVertical }\end{array}$ \\
\hline
\end{tabular}

SHELL GEOMETRY (Complete Sketch Below)

Inside Diameter, (in.) 57,3

Wall Thickness, (in.) Material

Inlet Nozzle ID, (in.) $\quad 22.8$

Outlet Nozzle ID, (in.) 22,8

Impingement Protection ${ }^{\prime}$ No $\square$ Yes

(Describe)

Annular Distributor No QYes

Open Cut Area $m^{2}$ (in. $^{2}$ )

Nozzle-to-First Tube Row Distance, (in.) 1.87

\section{CROSS BAFFLE GEOMETRY}

Type Segmental; $\square$ Double-Segmental $\square$ Disc/Doughnut

$\square$ Triple-Segmental; $\square$ No-Tubes-in-Window

Baffle Cut, \% Shell Diameter 44

Cut Orientation Relative to Axis of Inlet Nozzle

Inlet Baffle $\triangle$ Perpendicular $\square$ Parallel $\square 45^{\circ}$

Central Baffles Perpendicular o Parallel $045^{\circ}$

Baffle Thickness, (in.) 0,63 Material

Diametral Clearances Shell-to-baffle, (in.) 0.27

Tube-to-baffle (in.) 0.02

Bundle-to-shell, (in.) 3.74

Number of Baffles Along Length of Shell

Baffle Spacing, (in.) ___ Central 34.49 Inlet 40.1 Outlet 34.34

Unsupported Tube Span Lengths, (in.)

Longest 74.6 Inlet 74.6 Outlet 68.82

\section{TUBE GEOMETRY}

Outside Diameter, $\operatorname{mm}$ (in.) $\quad 0.988$

Wall Thickness, (in.) 0.07874 Material CS

Tube Lengths

Straight Tube, Inside Tubesheets, (in.) 143.48

U-Tube, Tubesheet to Bend Tangent, (in.) -

Tube Pitch, (in.) $\quad 1.26$

Layout (Please Circle)
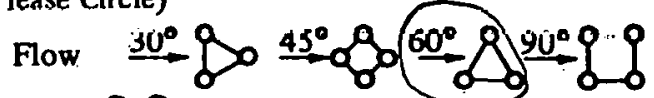

No. of Tubes 1508 No. of Tubepasses 4

First Tubepass $\square$ Countercurrent $\square$ Cocurrent

Tubc-to-Tubesheet Joint

$\nabla$ Welded $\square$ Roller Expanded D Other

If U-Tube

Maximum Bend Radius, mm.(in.)

Bend Orientation Relative to Axis of Shellside Inlet Nozzle

a Perpendicular D Parallel

If Bend Supported, Describe in Comments Below.

If Finned Tubes

Fins/on (Fins/in.) —_ Fin Material _-

Diameter, (in.), Root __ Over Fins__ _

If Enhanced Surface Tubes

(Describe)

\section{DETUNING BAFFLE}

If Detuning Baffle Used to Control Acoustic

Vibration, Indicate Position on Sketch Below

\section{COMMENTS AND SKETCH}

Complete sketches by drawing in tubeslde and shellside nozzles.

Indicate inlet nozzles with an arrow.

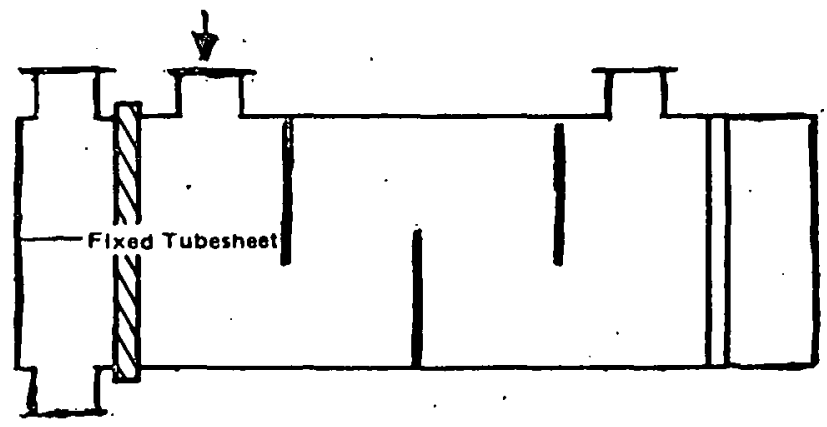

Show shellside inlet nozzle location, batfle cut orientation, and impingement devices.

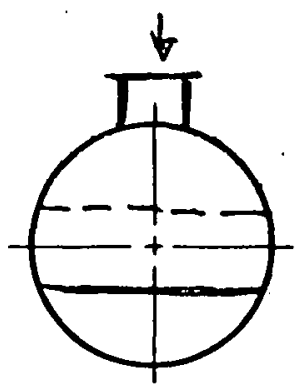


Assignèd

Case No. 106

PROCESS CONDITIONS

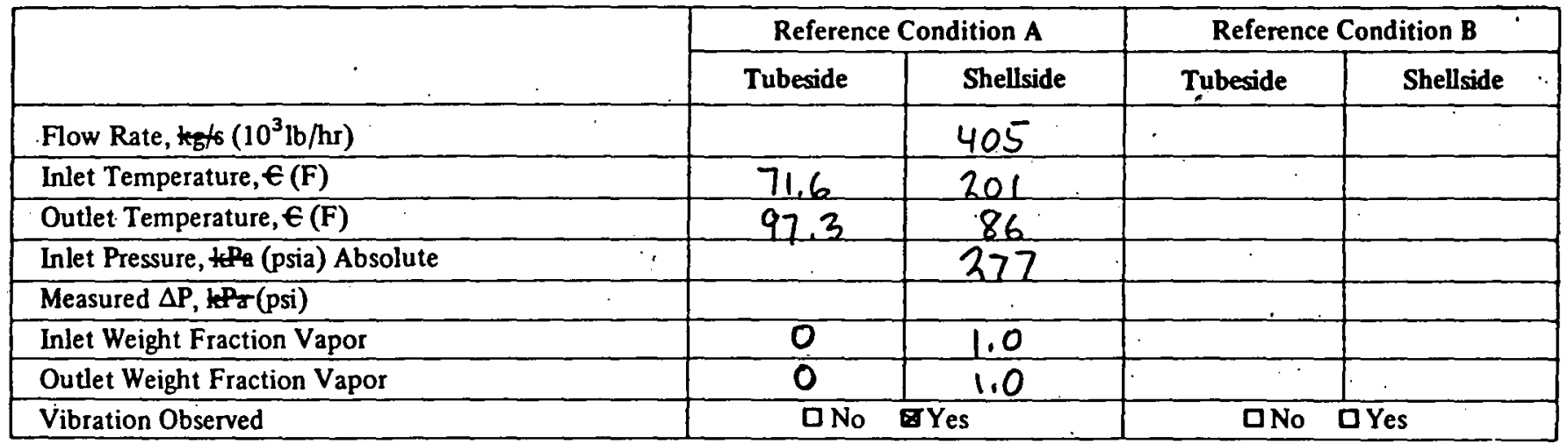

FLUID PHYSICAL PROPERTIES

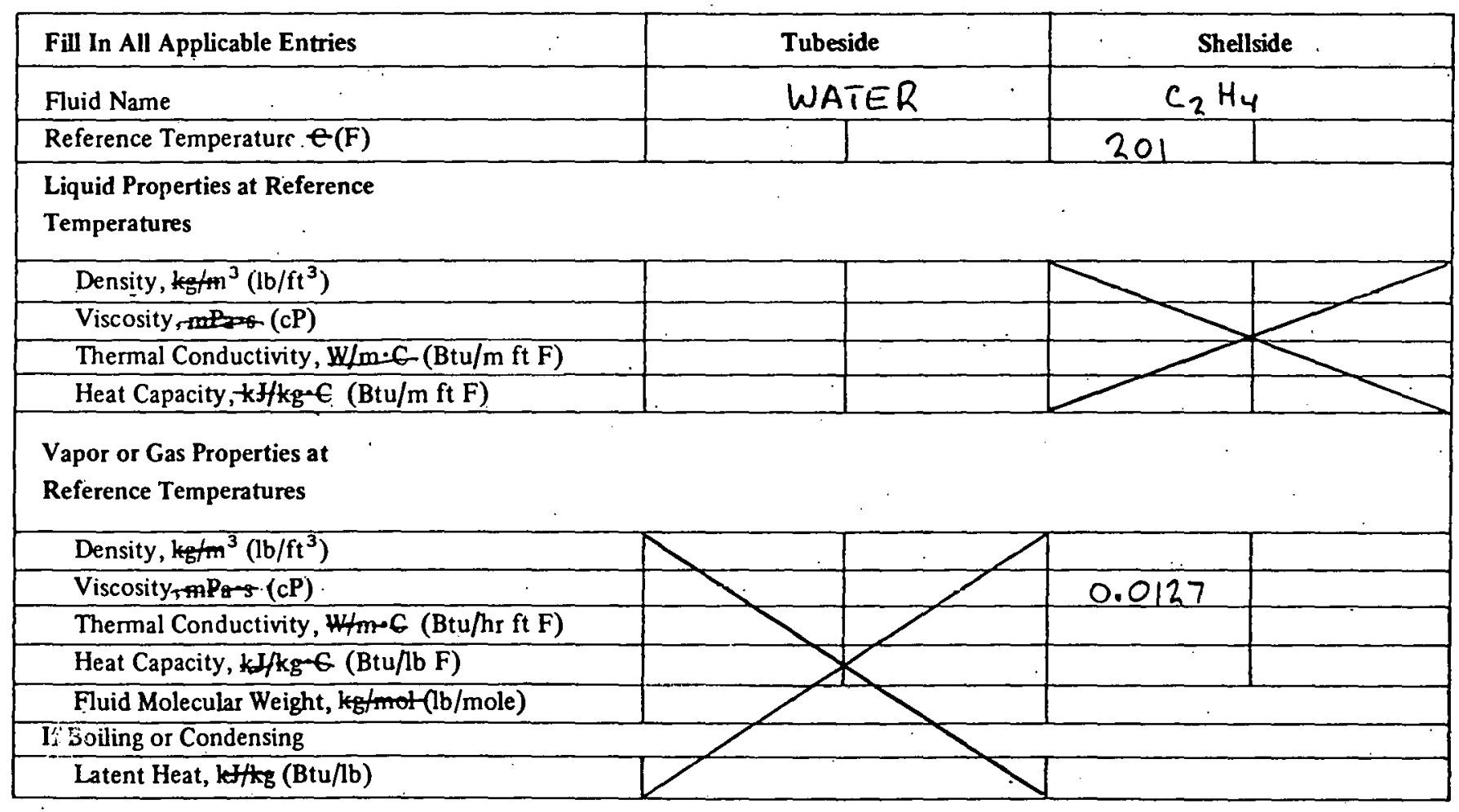




\begin{tabular}{ll|}
\hline Assigned & 106 \\
\hline
\end{tabular}

VIBRATION AND DAMAGE DESCRIPTION

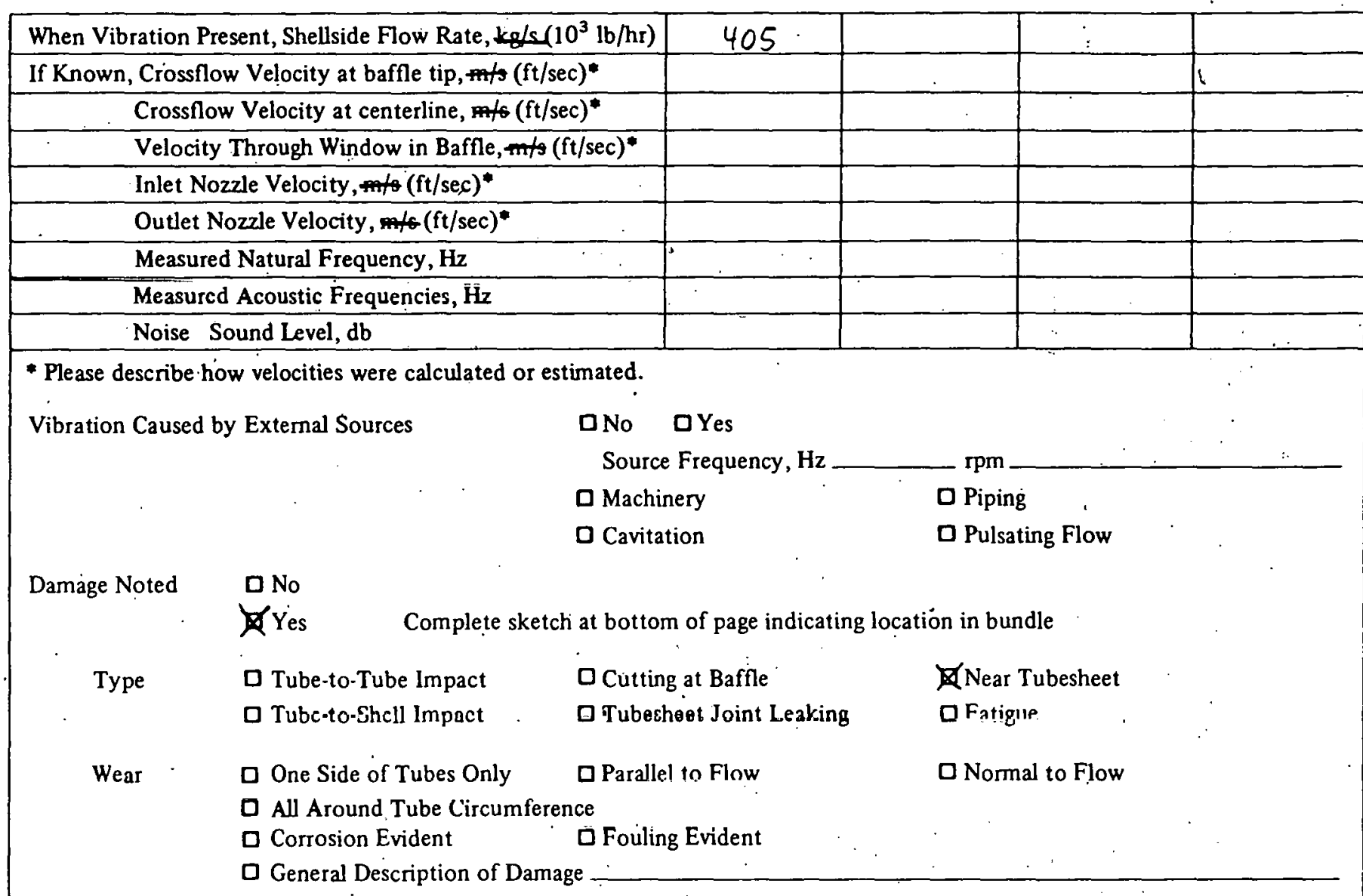

Exchanger Operation History

- How Long on Stream Before Damage Occurred?

- Any Unusual Occurrence Observed Prior to Vibration as a Consequence of
口Start-up
D Plant-Upset ·
a Shutdown

Describe

- If Vibration Remedy Afplied, Describe and Indirate Results:

TUBE BUNDLE DAMAGE SKETCH
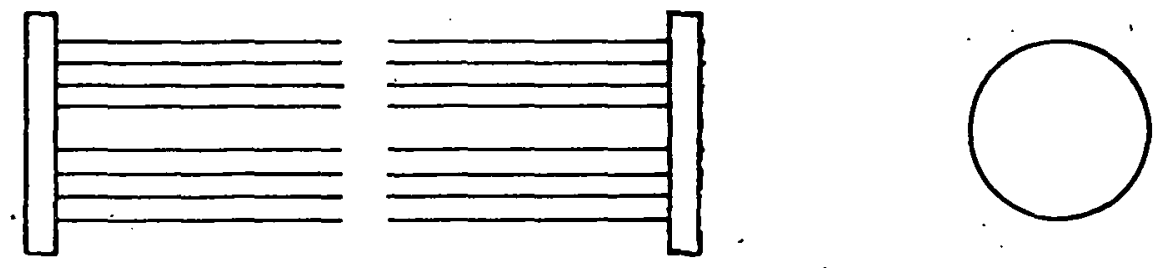

$2-26$ 
To protect the identity of the organization submitting this case, HTRI has assigned a case number. Additionally the data on pages 2, 3, and 4 have been reviewed to ensure that they do not include any propietary information.

This is a replacement for the original page 1 that provides space for

Assigned

Case No. additional comments, drawings, photographs, etc.

Summary

This 37-in. diameter by 40-ft long CEM TEMA style heat exchanger is one of two in series joined by welding their shells together. The tubeside fluid, natural gas with ethylene glycol to prevent hydration, passes from the one exchanger to the next without external piping. The shellside fluid, natural gas, flows counter current and between shells through external piping. Both exchangers were identical on the shell side and both experienced similar tube damage in the same locations of the exchanger. Most of the damage was noted after operating the plant at more than 40 percent higher than the designed shellside flow. Leaking tubes were plugged. During a subsequent overhaul of the bundles, the leaking tubes were found to be "broken on side. near the nozzles." It is interesting to note that the damaged tubes were not those with the longest span at the inlet end zone.

For this case, there are construction drawings and a field report describing the operation history and the repairs made to the exchanger. 


\begin{tabular}{|c|c|c|c|c|c|}
\hline & & & & $\begin{array}{l}\text { Assigned } \\
\text { Case No. } 1\end{array}$ & 07 \\
\hline Type & $\begin{array}{l}\text { TEMA Exchanger } \\
\text { Special Exchanger }\end{array}$ & $\begin{array}{l}\text { Designation CEM } \\
\text { (Describe) }\end{array}$ & FIXEO TUBESHEET & Shell Orientation & $\begin{array}{l}\text { QHorizontal } \\
\text { Q Vertical }\end{array}$ \\
\hline
\end{tabular}

\section{SHELL GEOMETRY (Complete Sketch Below)}

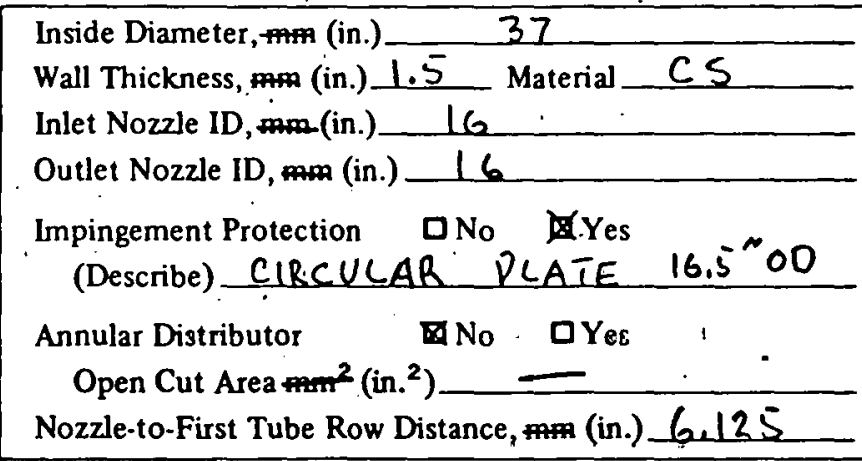

\section{CROSS BAFFLE GEOMETRY}

Type $\square$ Segmental; Double-Segmental $\square$ Disc/Doughnut $\square$ Triple-Segmental; $\square$ No-Tubes-in-Window Baffle Cut, \% Shell Diameter 30,3 (1 TURE OVER LAP) Cut Orientation Relative to Axis of Inlet Nozzle

Inlet Baffle 口Perpendicular \&Parallel $045^{\circ}$

Central Baffles $\square$ Perpendicular Parallel $\square 45^{\circ}$ Baffle Thickness, (in.) $0.62 \bar{\zeta}^{\circ}$ Material CS Diametral Clearances Shell-to-baffle, (in.) 0.1875 Tube-to-baffle (in) $\frac{0.0156}{1.500}$ Bundle-to-shell, (in.) 1.500

Number of Baffles Along Length of Shell 16 Baffle Spacting, (III.)__ Cêlllial 27.3 inlet 30.6 Outlet $\quad 30.6$

Unsupported Tube Span Lengths, (in.)

Longest 57.9 Inlet 57.9 Outlet 57.9

\section{TUBE GEOMETRY}

Outside Diameter, (in.) 0.750

Wall Thickness, (in.) 0.065 Material CS

Tube Lengths

Straight Tube, Inside Tubesheets, (in.) 467.1 U-Tube, Tubesheet to Bend Tangent, mm (in.) -

Tube Pitch, mm (in.) $\quad 0.9375$

Layout (Please Circle)

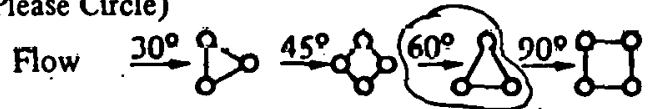

No. of Tubes $117 a$ No. of Tubepasses _................ First Tubepass Countercurrent $\square$ Cocurrent

Tube-to-Tubesheet Joint NOT GIVEN

$\square$ Welded $\square$ Roller Expanded DOther

If U-Tube

Maximum Bend Radius, mm (in.)

Bend Orientation Relative to Axis of Shellside Inlet Nozzle

$\square$ Perpendicular $\square$ Parallel

If Bend Supported, Describe in Comments Below

If Finned Tubes

Einstm (Fins/in.) _ Fin Material _ _

Diameter, (in.), Root - Over Fins -

If Enhanced Surface Tubes ___ _

(Describe)

\section{DETUNING BAFFLE}

If Detuning Baffle Used to Control Acoustic

Vibration, Indicate Position on Sketch Below

\section{COMMENTS AND SKETCH}

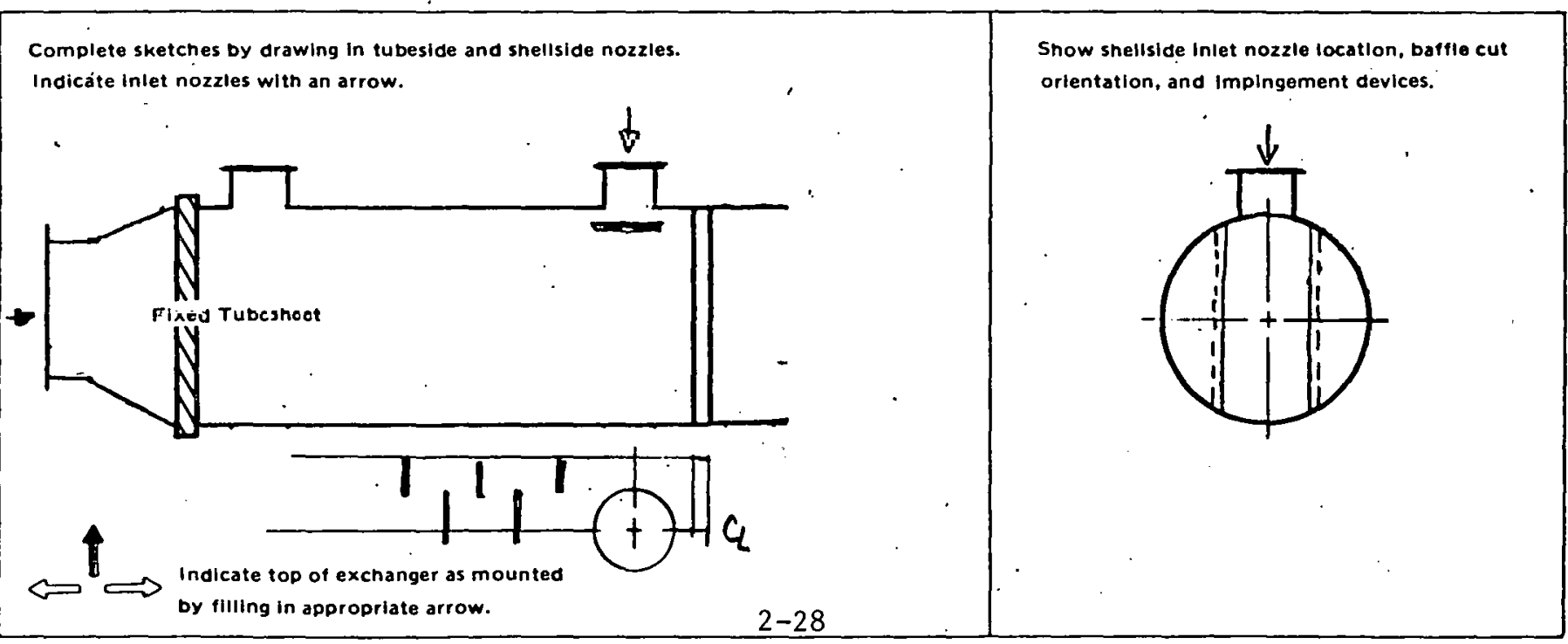


Page 3

\begin{tabular}{|l|}
\hline Assigned \\
Case No. 107 \\
\hline
\end{tabular}

\section{PROCESS CONDITIONS}

\begin{tabular}{|c|c|c|c|c|}
\hline & \multicolumn{2}{|c|}{ Reference Condition A } & \multicolumn{2}{|c|}{ Reference Condition B } \\
\hline & Tubeside & Shellside & Tubeside & Shellside \\
\hline Flow Rate, kfs $\left(10^{3} \mathrm{lb} / \mathrm{hr}\right)$ & - & 341.5 & & 210 \\
\hline Inlet Temperature, $\in(F)$ & 69 & -23 & 86 & -29 \\
\hline Outlet Temperature, $\in(\mathrm{F})$ & 25 & 57 & 26 & 80 \\
\hline Inlet Pressure, $\$ \mathbf{P}_{\mathbf{a}}$ (psia) Absolute & 1055 & 1045 & 10.50 & 1040 \\
\hline Measured $\Delta \mathrm{P}, k \mathbb{P}_{\mathbf{a}}(\mathrm{psi})$ & 10 & 20 & 5 & 8 \\
\hline Inlet Weight Fraction Vapor & 1.0 & 1.0 & 1.0 & 1.0 \\
\hline Outlet Weight Fraction Vapor & 1.0 & 1.0 & 1.0 & 1.0 \\
\hline Vibration Observed & \multicolumn{2}{|l|}{ QNo } & \multicolumn{2}{|c|}{ 及No DYes } \\
\hline
\end{tabular}

\section{FLUID PHYSICAL PROPERTIES}

\begin{tabular}{|c|c|c|}
\hline Fill In All Applicable Entries & Tubeside & Shellside \\
\hline Fluid Name & NATURAC GAS + GLYCOL & NATURAL GAS \\
\hline \multicolumn{3}{|l|}{ Reference Temperature C (F) } \\
\hline \multicolumn{3}{|l|}{ Liquid Properties at Reference } \\
\hline \multicolumn{3}{|l|}{ Temperatures } \\
\hline \multicolumn{3}{|l|}{ Density, $\mathrm{kg} / \mathrm{m}^{3}\left(\mathrm{lb} / \mathrm{ft}^{3}\right)$} \\
\hline \multicolumn{3}{|c|}{ Viscosity, $\mathrm{mPa} \cdot \mathrm{s}(\mathrm{cP})$} \\
\hline \multicolumn{3}{|c|}{ Thermal Conductivity, $\mathrm{W} / \mathrm{m} \cdot \mathrm{C}(\mathrm{Btu} / \mathrm{m} \mathrm{ft} \mathrm{F})$} \\
\hline \multicolumn{3}{|c|}{ Heat Capacity, $\mathrm{kJ} / \mathrm{kg} \cdot \mathrm{C}(\mathrm{Btu} / \mathrm{m} \mathrm{ft} \mathrm{F})$} \\
\hline \multicolumn{3}{|c|}{$\begin{array}{l}\text { Vapor or Gas Properties at } \\
\text { Reference Temperatures }\end{array}$} \\
\hline \multicolumn{3}{|l|}{ Density, $\mathrm{kg} / \mathrm{m}^{3}\left(\mathrm{lb} / \mathrm{ft}^{3}\right)$} \\
\hline \multicolumn{3}{|c|}{ Viscosity, $\mathrm{mPa} \cdot \mathrm{s}(\mathrm{cP})$} \\
\hline \multicolumn{3}{|c|}{ Thermal Conductivity, $\mathrm{W} / \mathrm{m} \cdot \mathrm{C}(\mathrm{Btu} / \mathrm{hr} \mathrm{ft} \mathrm{F})$} \\
\hline \multicolumn{3}{|c|}{ Heat Capacity, $\mathrm{kJ} / \mathrm{kg} \cdot \mathrm{C}(\mathrm{Btu} / \mathrm{lb} \mathrm{F})$} \\
\hline \multicolumn{3}{|c|}{ Fluid Molecular Weight, $\mathrm{kg} / \mathrm{mol}(\mathrm{lb} / \mathrm{mole})$} \\
\hline \multicolumn{3}{|c|}{ If Boiling or Condensing } \\
\hline Latent Heat, $\mathrm{kJ} / \mathrm{kg}(\mathrm{Btu} / \mathrm{lb})$ & $=$ & \\
\hline
\end{tabular}




\begin{tabular}{|l|l|}
\hline Assigned & Page 4 \\
Case No. $\quad 107$ \\
\hline
\end{tabular}

\section{VIBRATION AND DAMAGE DESCRIPTION}

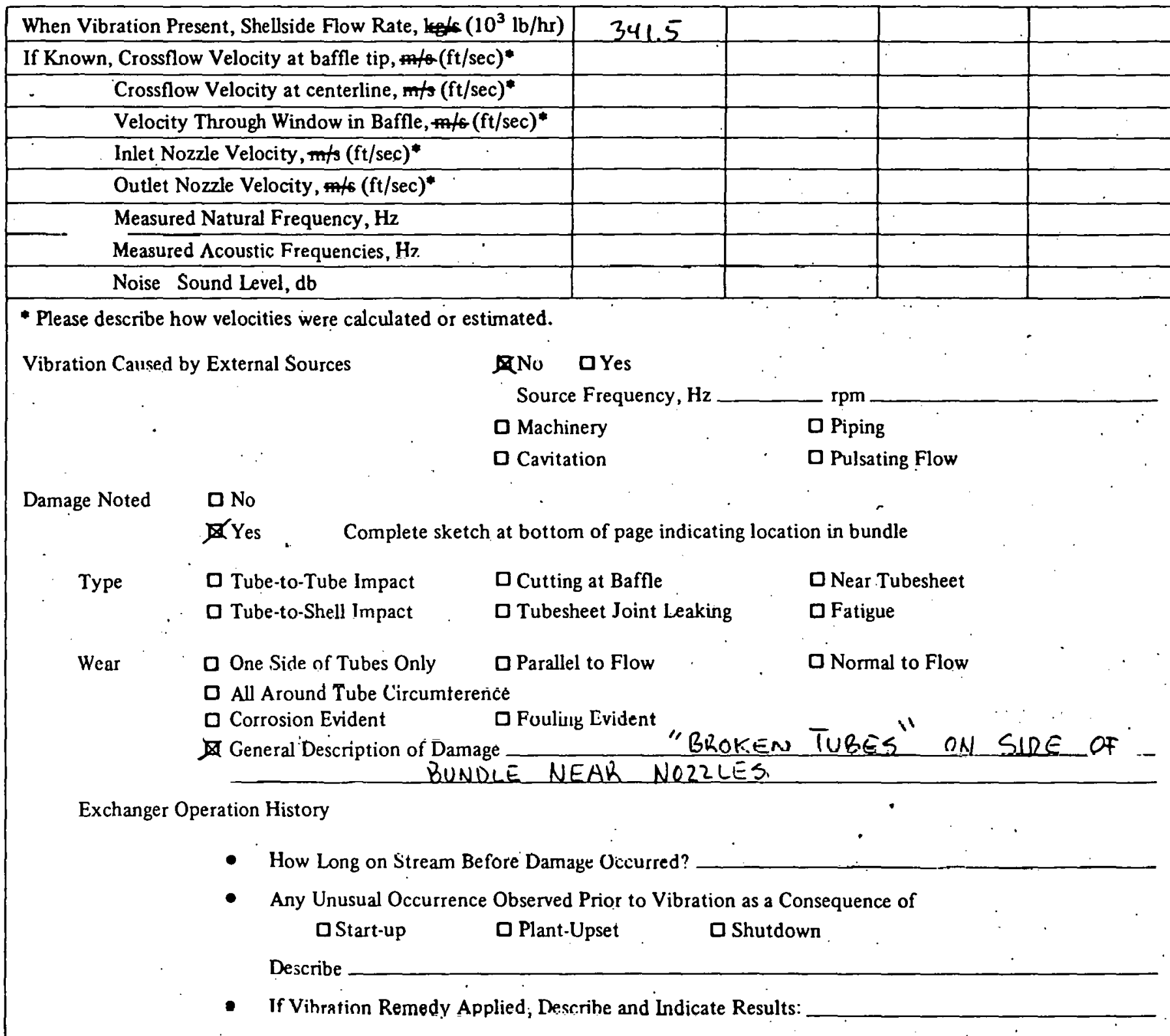

\section{TURE BUNDLE DAMAGE SKETCH}
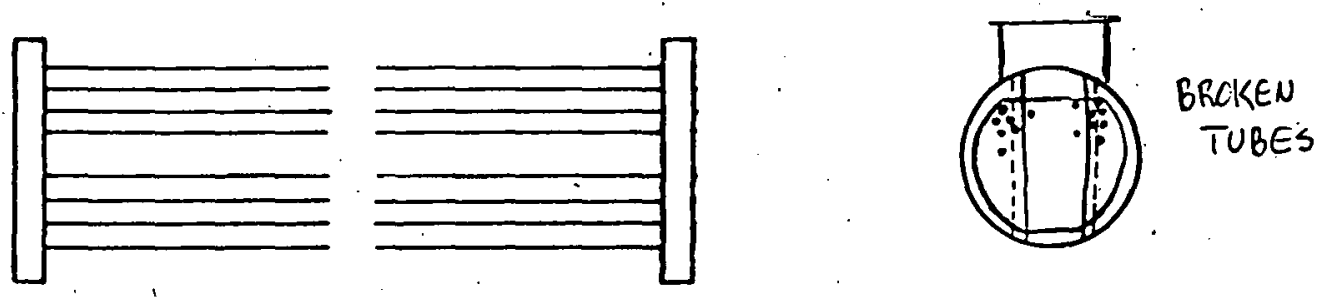
To protect the identity of the organization submitting this case, HTRI has assigned a case number. Additionally the data on pages 2, 3, and 4 have been reviewed to ensure that they do not include any propietary information.

Assigned

Case No.

This is a replacement for the original page 1 that provides space for additional comments, drawings, photographs, etc.

\section{Summary}

This 44-in. diameter by 40-ft long CEM TEMA style heat exchanger is one of three that are joined tubesheet to tubesheet so that in effect the tubes are $120 \mathrm{ft}$ long. The tubeside flow is natural gas with glycol to prevent hydration while the shellside flow is counter current and passes between shells through external piping. The bundle uses triple-segmental baffles so the shellside flow is primarily axial. The tube spans are longer than TEMA standards maximum spans for $3 / 4-i n$. carbon steel. The heat exchanger operated successfully for one and a half years at the design conditions. However, tubes began leaking where they were joined to the tubesheet in all three exchangers when the shellside flow rate was substantially increased beyond that used for design. The sketch below indicates the location of, these failures. The failed tubes were characterized by particularly long spans in the outlet end zones. Minor cutting of the tubes at the baffles was also reported.

For this case, there are construction drawings and a field report describing the operating history, the damage noted, and the repairs made to the exchanger.

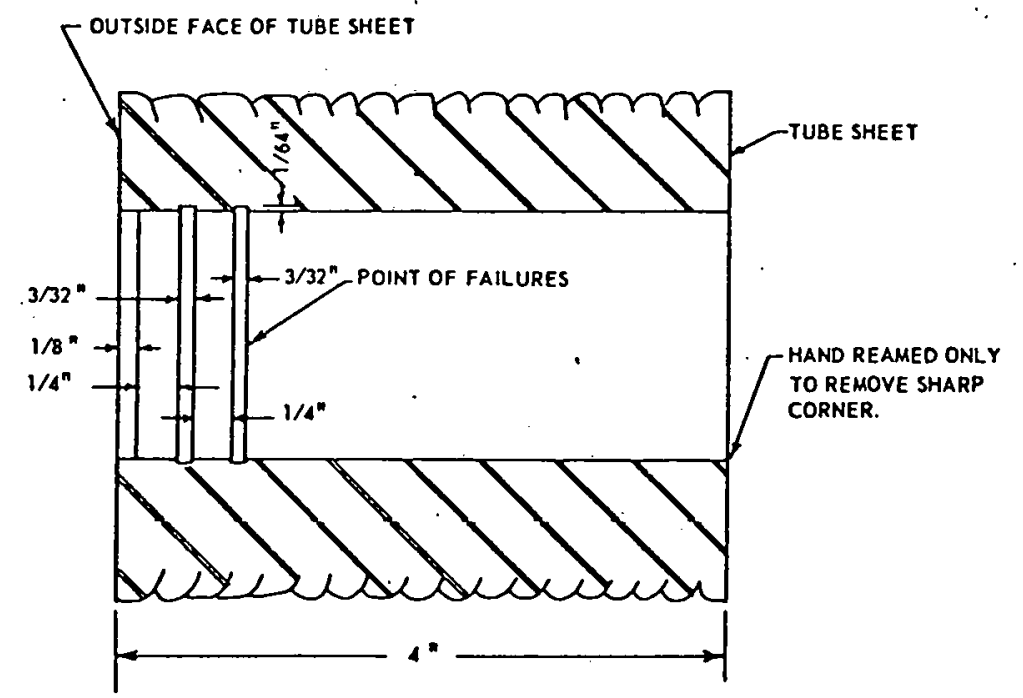




\begin{tabular}{l} 
Assigned \\
Case No. 108 \\
\hline
\end{tabular}

PROCESS CONDITIONS

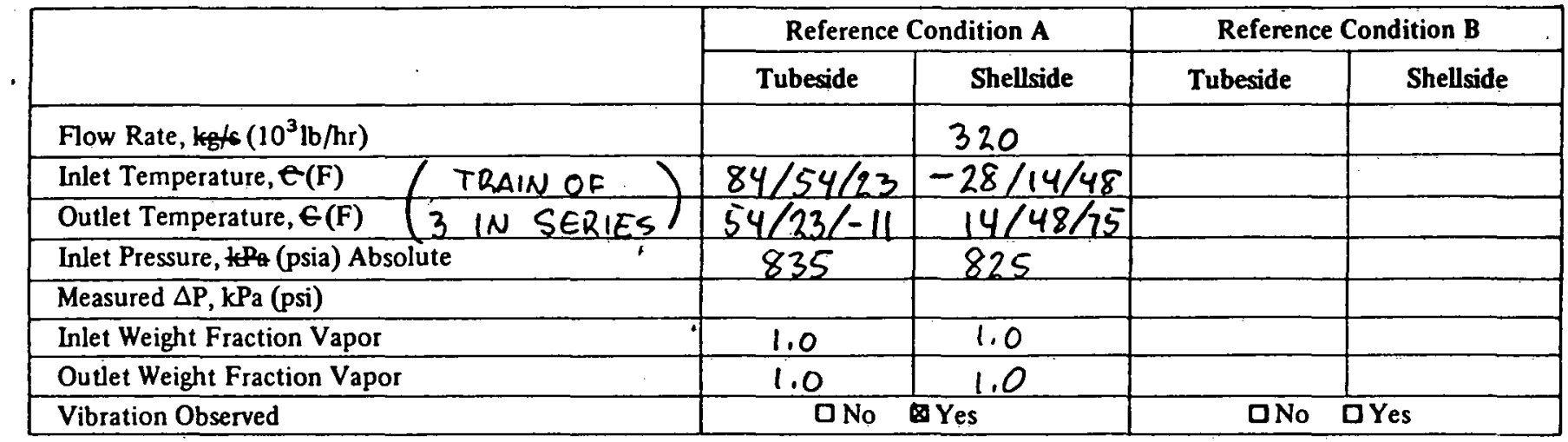

\section{FLUID PHYSICAL PROPERTIES}

\begin{tabular}{|c|c|c|c|c|}
\hline Fill In All Applicable Entries & \multicolumn{2}{|c|}{ Tubeside } & \multicolumn{2}{|c|}{ Shellside } \\
\hline Fluid Name & \multicolumn{2}{|c|}{ NAT'L GAS + GLYCOL } & \multicolumn{2}{|c|}{ NAT'C GAS } \\
\hline Reference Temperature $€(F)$ & 84 & -11 & -28 & 75 \\
\hline \multicolumn{5}{|l|}{$\begin{array}{l}\text { Liquid Properties at Reference } \\
\text { Temperatures }\end{array}$} \\
\hline \multicolumn{5}{|l|}{ Density, $\mathrm{kg} / \mathrm{m}^{3}\left(\mathrm{lb} / \mathrm{ft}^{3}\right)$} \\
\hline \multicolumn{5}{|l|}{ Viscosity, $\mathrm{mPa} \cdot \mathrm{s}(\mathrm{cP})$} \\
\hline \multicolumn{5}{|l|}{ Thermal Conductivity, $\mathrm{W} / \mathrm{m} \cdot \mathrm{C}(\mathrm{Btu} / \mathrm{m} \mathrm{ft} \mathrm{F})$} \\
\hline \multicolumn{5}{|l|}{ Heat Capacity, $\mathrm{kJ} / \mathrm{kg} \cdot \mathrm{C}(\mathrm{Btu} / \mathrm{m} \mathrm{ft} F)$} \\
\hline \multicolumn{5}{|l|}{$\begin{array}{l}\text { Vapor or Gas Properties at } \\
\text { Reference Temperatures }\end{array}$} \\
\hline Density, ${ }^{3}\left(\mathrm{lb} / \mathrm{ft}^{3}\right)$ & 2.79 & 3.86 & 3,82 & 2.62 \\
\hline \multicolumn{5}{|l|}{ Viscosity, mPer (cP) } \\
\hline \multicolumn{5}{|l|}{ Thermal Conductivity, $W / m(B t u / h r f t F)$} \\
\hline \multicolumn{5}{|l|}{ Heat Capacity, $\mathrm{k} / \mathrm{kg} \in(\mathrm{Btu} / \mathrm{lb} \mathrm{F})$} \\
\hline \multicolumn{5}{|l|}{ Fluid Molecular Weight, $\mathrm{kg} / \mathrm{mol}$ (lb/mole) } \\
\hline \multicolumn{5}{|l|}{ It Boiling or Condensing } \\
\hline Latent Heat, $\mathrm{K}_{\mathrm{K}} \mathrm{kg}(\mathrm{Btu} / \mathrm{lb})$ & & & & \\
\hline
\end{tabular}




\begin{tabular}{|l|}
\hline Assigned \\
Case No. 108 \\
\hline
\end{tabular}

VIBRATION AND DAMAGE DESCRIPTION

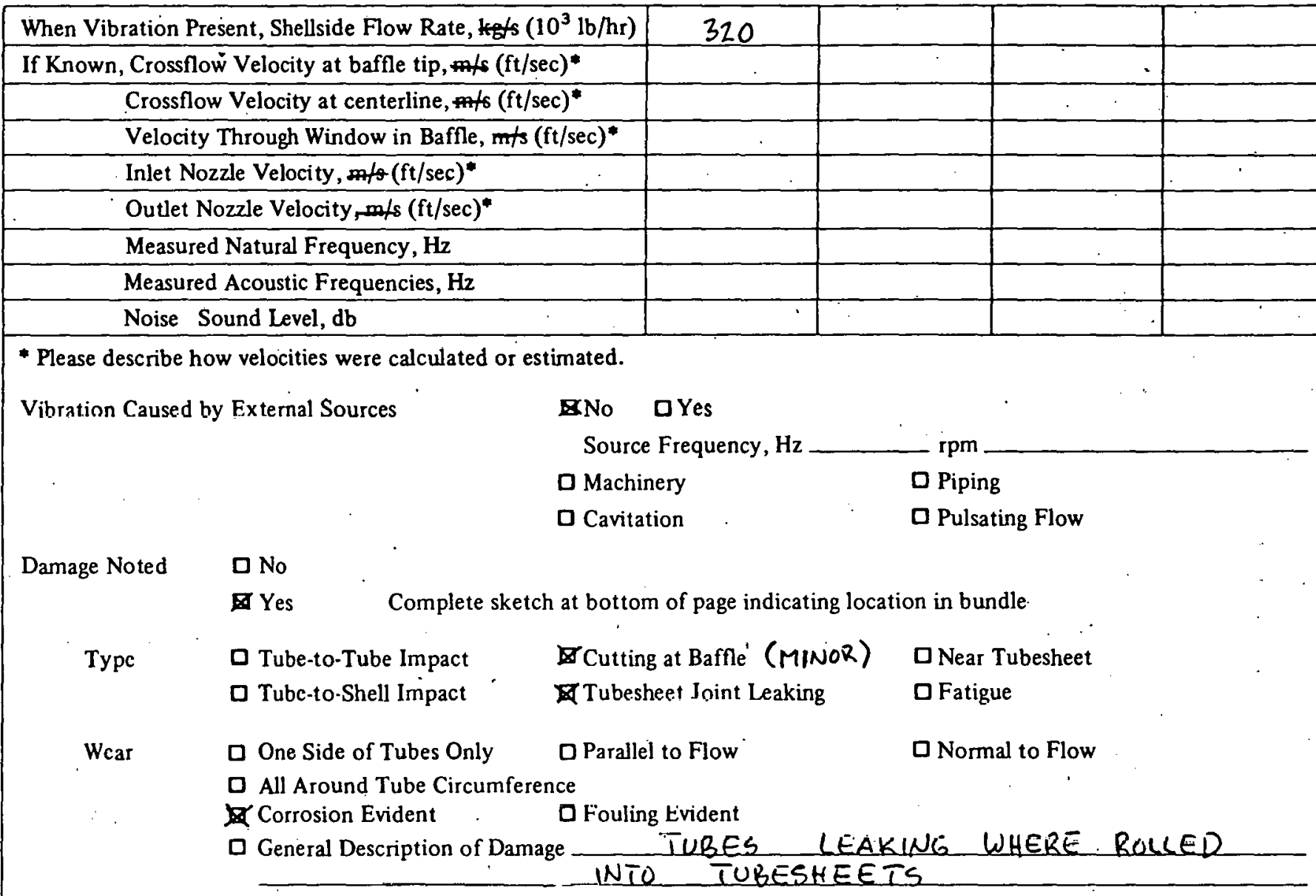

Exchanger Operation History

- How Long on Stream Before Damage Occurred? 1 1 _ Y $\frac{1}{2}$ EARS

- Any Unusual Occurrence Observed Prior to Vibration as a Consequence of
口Start-up
$\square$ Plant-Upset
$\square$ Shutdown

Describe INCREASED FLOWRATE BEYOND DESIGN

- If Vibration Remedy Applied, Doscribe and Indirate Results.

TUBE BUNDLE DAMAGE SKETCH
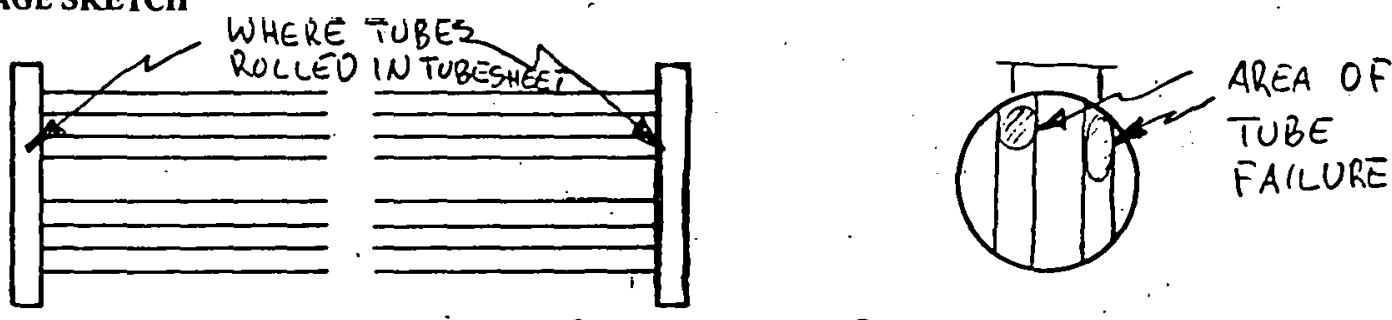

most fallures in SHELLS A AND B.

$2-34$ 
To protect the identity of the organization submitting this case, HTRI has assigned a case number. Additionally the data on pages 2, 3, and 4 have been reviewed to ensure that they do not include any propietary information.

This is a replacement for the original page 1 that provides space for additional comments, drawings, photographs, etc.

\section{Summary}

This 53-in. diameter by 40-ft long CEM TEMA style heat exchanger is one of two in series that share a common shell. There is natural gas with glycol to prevent hydration on the tube side. Between exchangers the tubeside flow passes through an open shell section about $4 \mathrm{ft}$ long before entering the tubes of the downstream exchanger. The sheliside fluid is natural gas flowing counter current and piped externally between shells. The bundle has double-segmented baffles and is flat on top to a depth of 8-1/2 in. A round impingement plate the same diameter as the inlet nozzle ID is $8-1 / 2$ in. below the nozzle. A unique feature is a flat plate seal welded to the shell to block shellside flow bypassing at the top of the bundle. The maximum span lengths are less than the TEMA Standards maximum lengths for $3 / 4-$ in. carbon steel tubes.

No tube vibration has been noted in this unit even when operated at greater than 20 percent over the designed shellside flow rate. For this case, there are construction drawings and a short field report of the operating history of the exchanger train. 


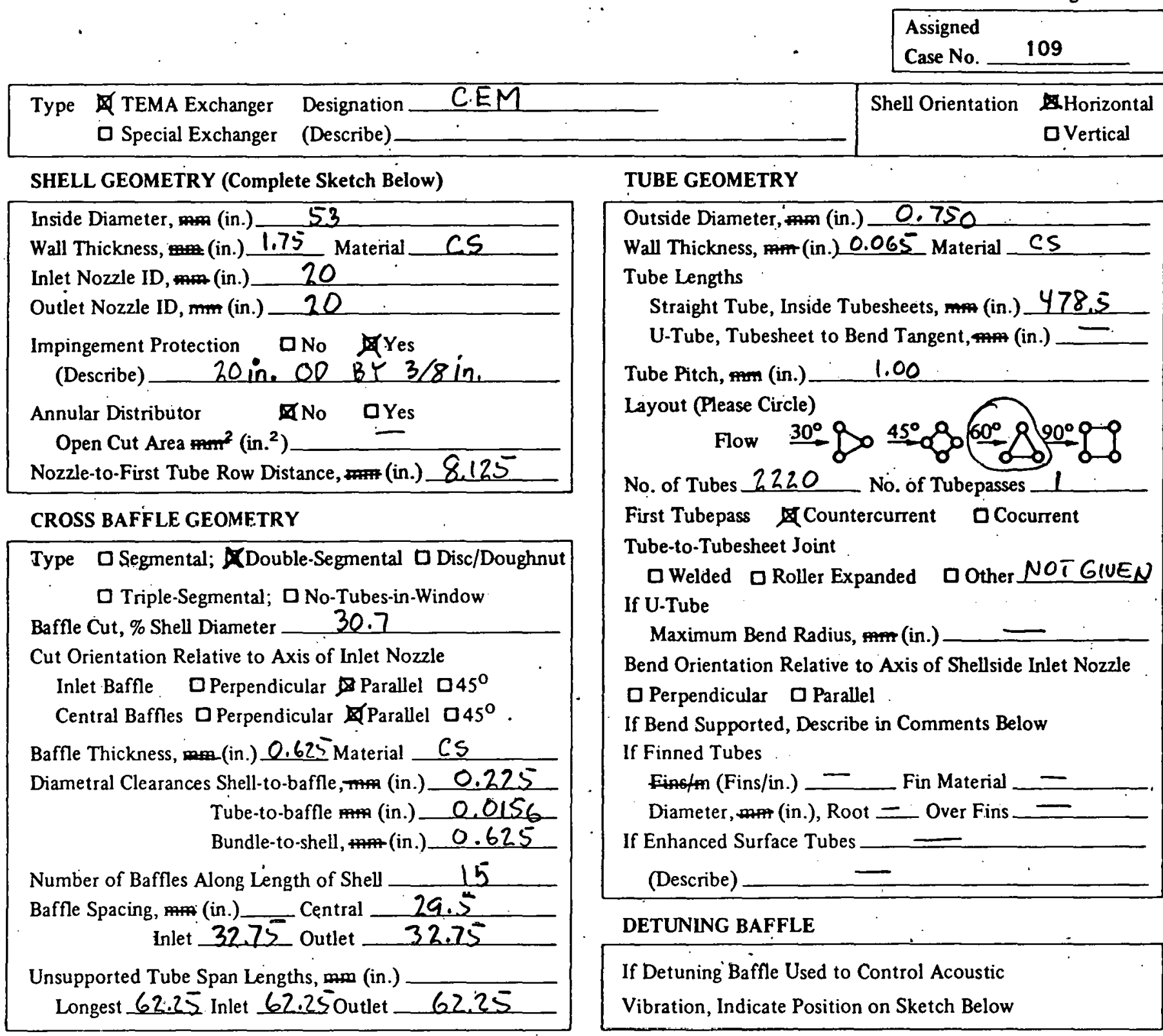

\section{COMMENTS AND SKETCH}

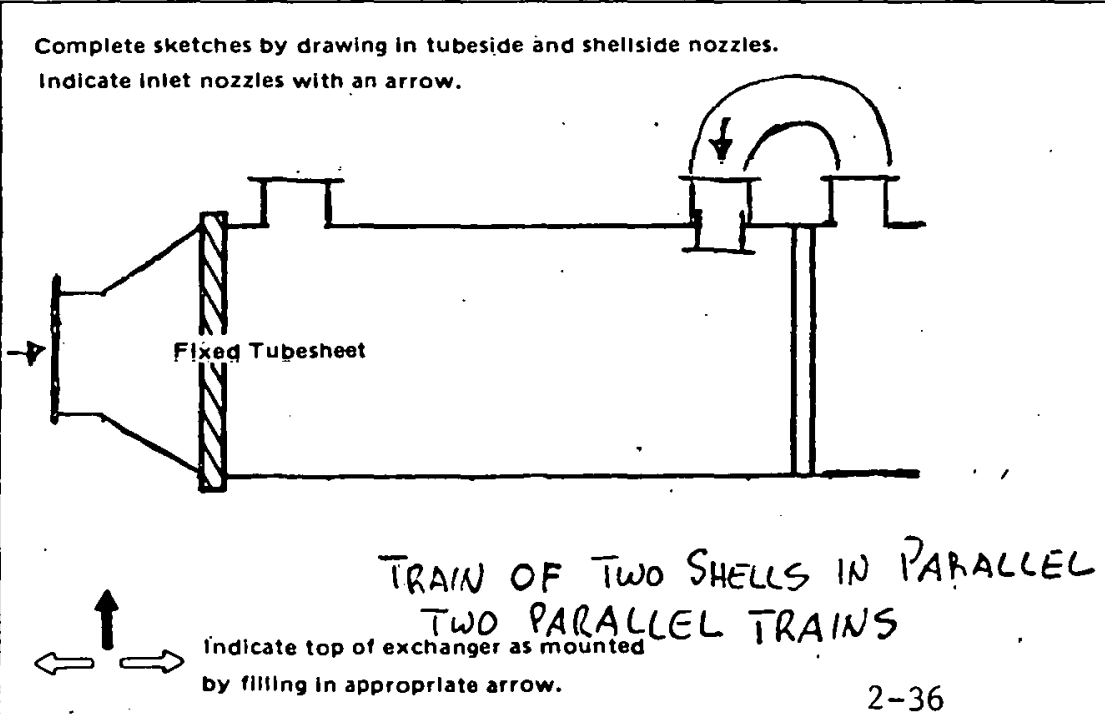

Show shellside inlet nozzle location, baffle cut orientotion, and impingement devices.

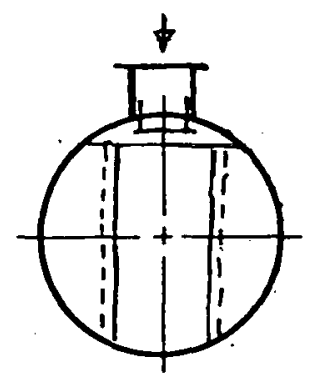

Train of Two Shells in Parallel TWO PARALLEL TRAINS by flliling in approprlate arrow. 


\begin{tabular}{|l|}
\hline Assigned \\
Case No. 109 \\
\hline
\end{tabular}

\section{PROCESS CONDITIONS}

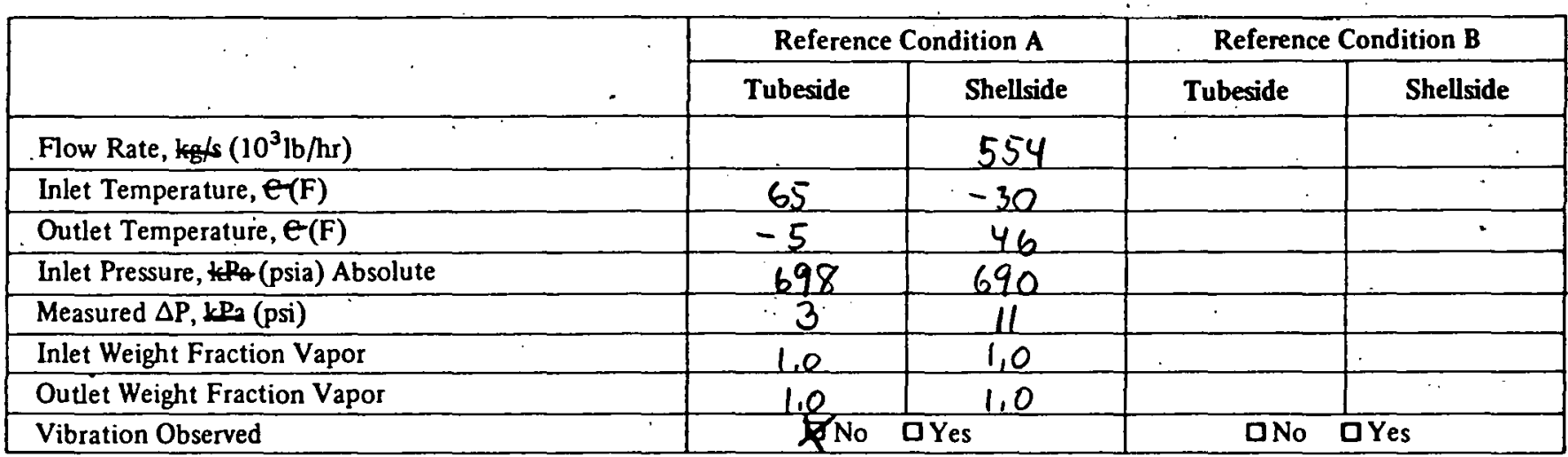

\section{FLUID PHYSICAL PROPERTIES}

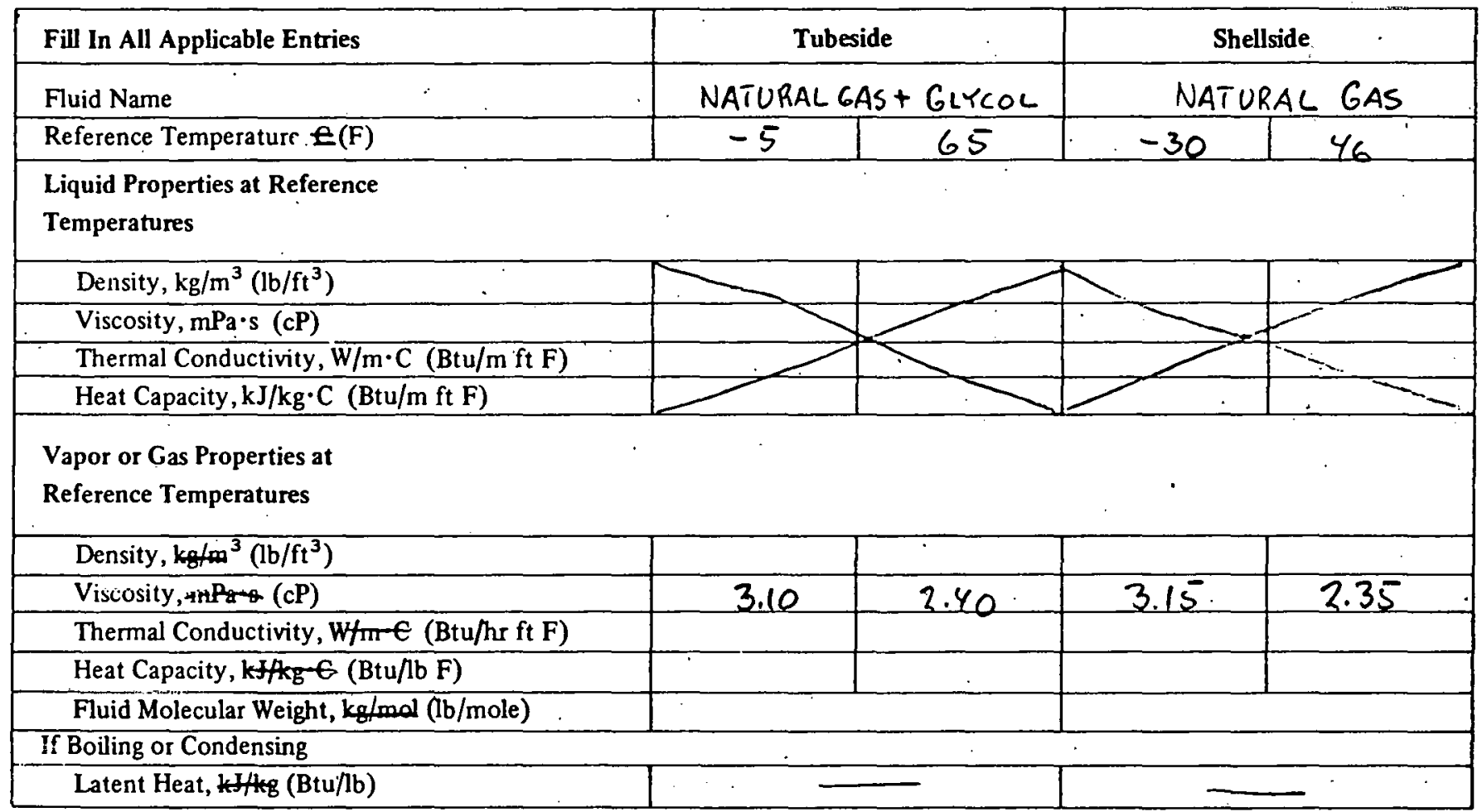




\section{NO VIBRATION OBSERVED}

\begin{tabular}{|l|}
\hline Assigned \\
Case No. 109 \\
\hline
\end{tabular}

VIBRATION AND DAMAGE DESCRIPTION

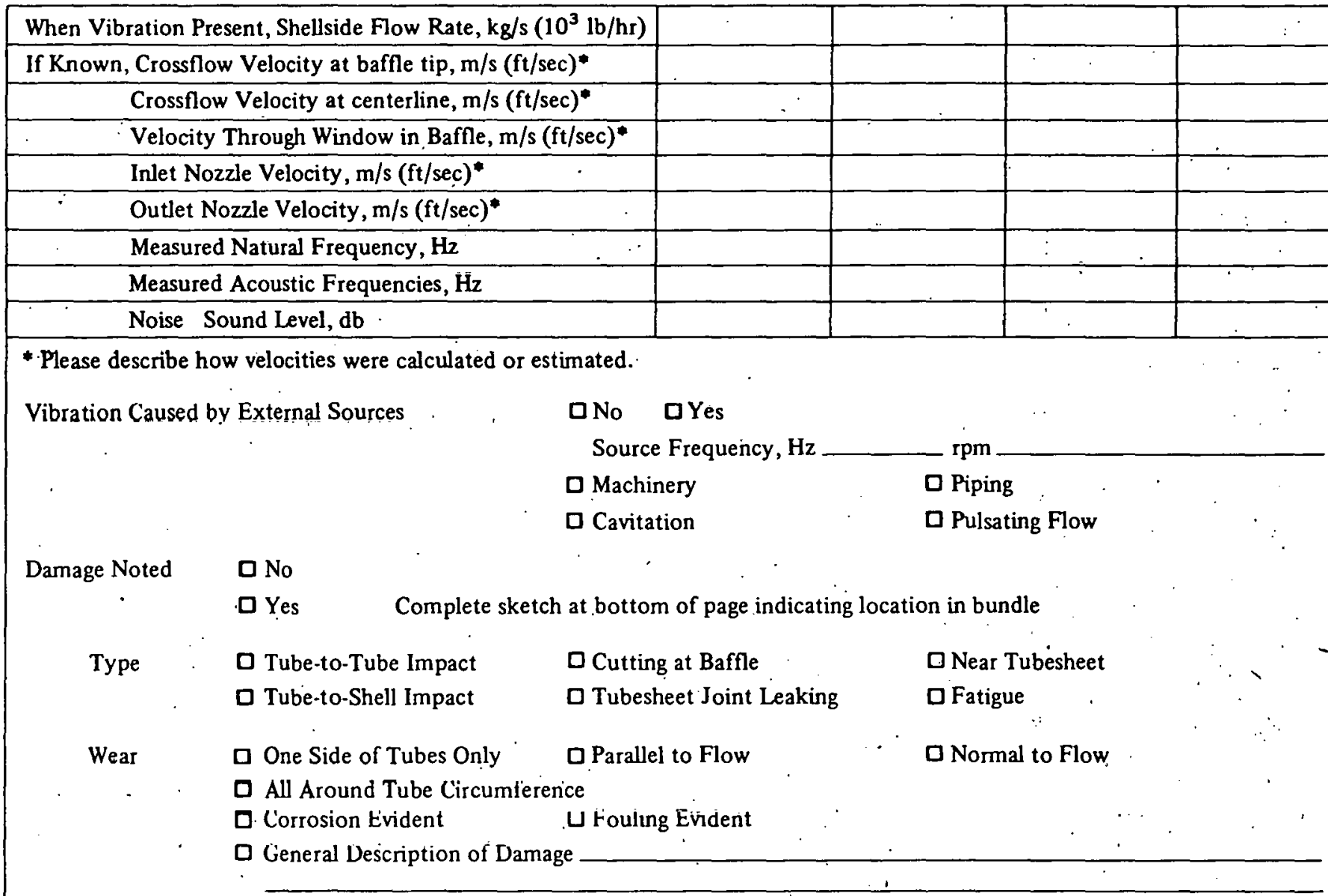

Exchanger Operation History

- How Long on Stream Before Damage Occurred?

- Any Unusual Occurrence Observed Prior to Vibration as a Consequence of
D Start-up
D. Plant-Upset
口 Shutdown

Describe

- If Vibration Remedy Applied, Describe and Indicate Results:

TUBE BUNDLE DAMAGE SKETCH
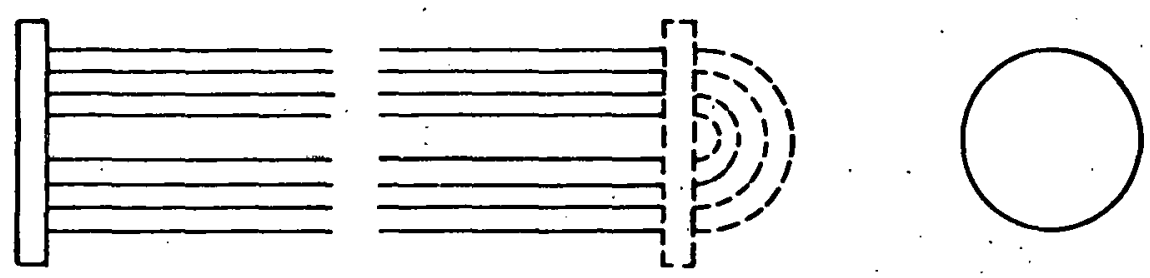
To protect the identity of the organization submitting this case, HTRI has assigned a case number. Additionally the data on pages 2, 3, and 4 have been reviewed to ensure that they do not include any propietary information.

This is a replacement for the original page 1 that provides space for Assigned Case No. 110 additional comments, drawings, photographs, etc.

Summary

This 45-in, diameter by 40-ft long CEM TEMA style heat exchanger is in refinery service. The tubeside fluid is cooling tower water while the shellside fluid is an olefin-isooctane mixture with hydrofluoric acid. Indications are that there was a loud noise coming from the exchanger at. start-up before the tubeside fluid was brought on stream. Tubes that failed were those within a few rows of the baffle tips of the double-segmental baffles. The breaks were at baffles near the center of the tube length. Metallurgical analysis indicates corrosion fatigue that was probably initiated by flow-induced vibration. The exchanger bundle was replaced with a new one with 18 instead of 12 baffles and no further vibration was noted (See Case 111).

For this case, there are partial construction drawings, an indication of the specific tubes that failed, and a metallurgical report on the failed tubes. 


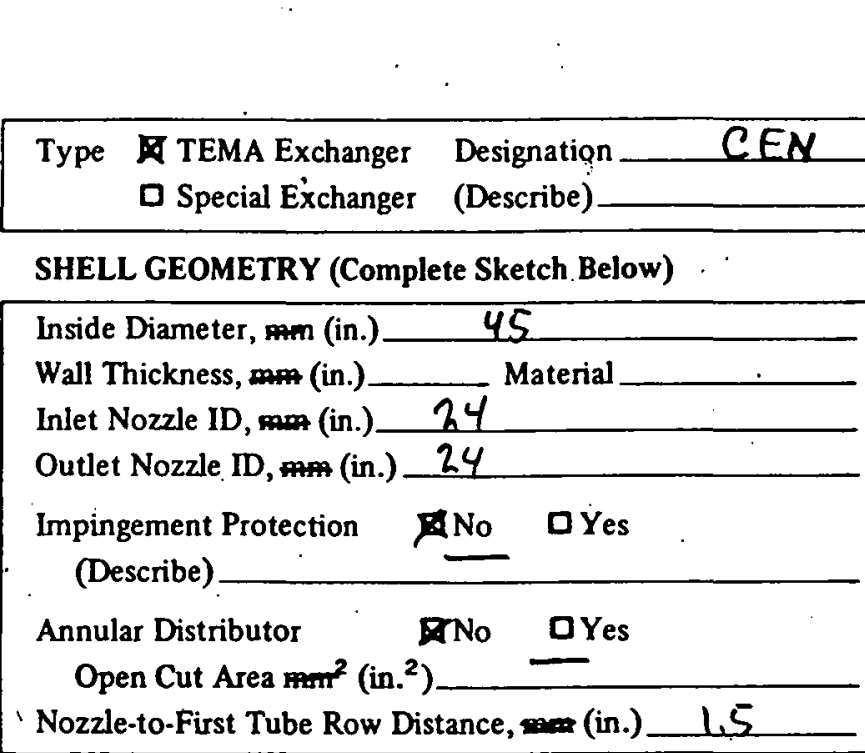

\section{CROSS BAFFLE GEOMETRY}

Type $\square$ Segmental; $\mathbf{X}$ Double-Segmental $\square$ Disc/Doughnut

口 Triple-Segmental; $\square$ No-Tubes-in-Window

Baffle Cut, \% Shell Diameter 36.5

Cut Orientation Relative to Axis of Inlet Nozzle

Inlet Baffle 口Perpendicular Parallel $\square 45^{\circ}$

Central Baffles $\square$ Perpendicular Parallel $\square 45^{\circ}$

Baffle Thickness, (in.) 0.625Material CS

Diametral Clearances Shell-to.baffle, (in.) 0.25

Tube-to-baffle (in.) 0.0156

Bundle-to-shell, (in.) 1.125

Number of Baffles Along Length of Shell 12

Baffle Spacing, (in.) Central 36.5

$$
\text { Inlet } 35.9 \text { Outlet } 35.9
$$

Unsupported Tube Span Lengths, (in.)

Longest 70.4 Inlet 70.4 Outlet 70.4

\section{Assigned}

Case No.

110

Shell Orientation

Rorizontal QVertical

\section{TUBE GEOMETRY}

Outside Diameter, (in.) 1.0

Wall Thickness, (in.) 0.134 Material CS

Tube Lengths

Straight Tube, Inside Tubesheets, (in.) 473.25

U.Tube, Tubesheet to Bend Tangent, mm (in.) _ -

Tube Pitch, mm(in.) 1.25

Layout (Please Circle)

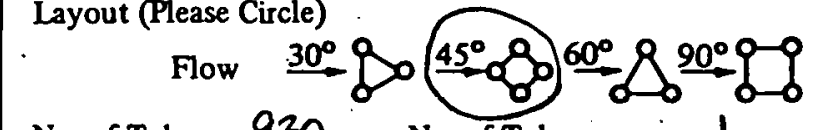

No. of Tubes 930 No. of Tubepasses

First Tubepass Countercurrent $\square$ Cocurrent

Tube-to-Tubesheet Joint

D. Welded Roller Expanded Dother

If U-Tube

Maximum Bend Radius, (in.)

Bend Orientation Relative to Axis of Shellside Inlet Nozzle

a Perpendicular a Parallel

If Bend Supported, Describe in Comments Below

If Finned. Tubes

Finotm (Fins/in.) _ _ Fin Material ___

Diameter, mm (ifr), Root ___ Over Fins ___ _

If Enhanced Surface Tubes ___ _ _ _

(Describe)

DE I UNING BAFFLE

If Detuning Baffle Used to Control Acoustic

Vibration, Indicate Position on Sketch Below

\section{COMMENTS AND SKETCH}

Complete sketches by drawing in tubeslde and shellside nozzles.

Indicate inlet nozzles with an arrow.

Show shellside inlot nozzle location, baffle cut , orlentation, and impingement devices.
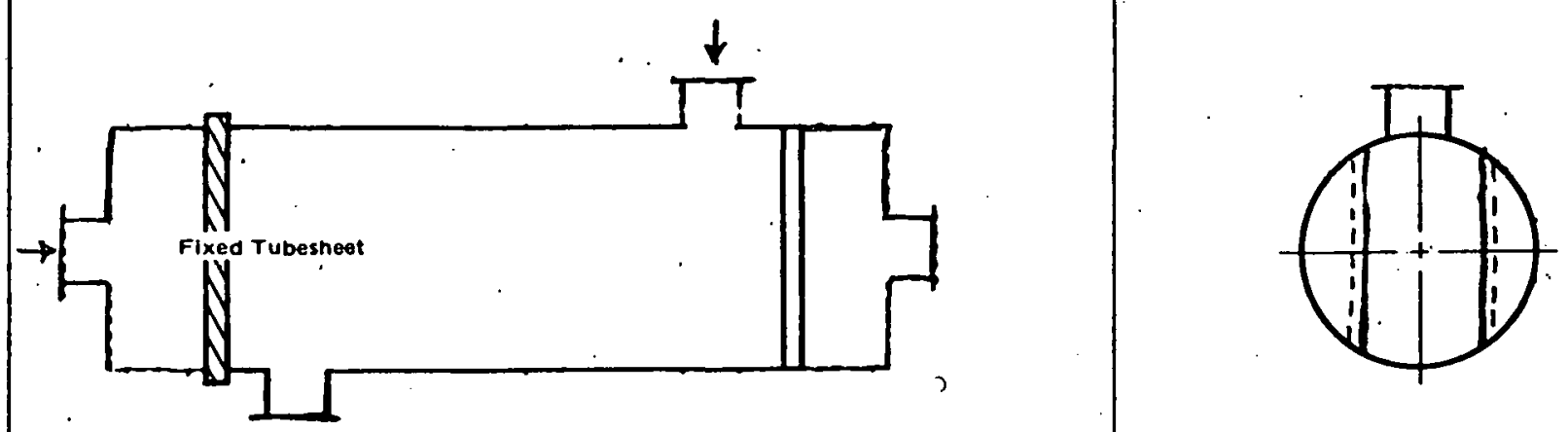

$\leftrightarrow \leftrightarrow$ 


\section{PROCESS CONDITIONS}

\begin{tabular}{|c|c|c|c|c|c|}
\hline & \multicolumn{2}{|c|}{ Reference Condition A } & \multicolumn{2}{|c|}{ Reference Condition B } \\
\hline & & Tubeside & Shellside & Tubeside & Shellside \\
\hline Flow Rate, $\mathrm{kg} / \mathrm{c}\left(10^{3} \mathrm{lb} / \mathrm{hr}\right)$ & & & 3300 & & \\
\hline Inlet Temperature, $\in(F)$ & & & 95 & & \\
\hline Oitlet Temperature, $€(F)$ & & & 90 & & \\
\hline Inlet Pressure, ${ } \mathbb{P}_{a}$ (psia) Absolute & 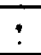 & & 200 & & \\
\hline Measured $\Delta \mathrm{P}, \mathrm{kP}_{\mathrm{Q}}$ (psi) & & & - & & \\
\hline Inlet Weight Fraction Vapor & & 0 & 0 & & \\
\hline Outlet Weight Fraction Vapor & & 0 & 0 & & \\
\hline Vibration Observed & & DNo & & aNo & \\
\hline
\end{tabular}

\section{FLUID PHYSICAL PROPERTIES}

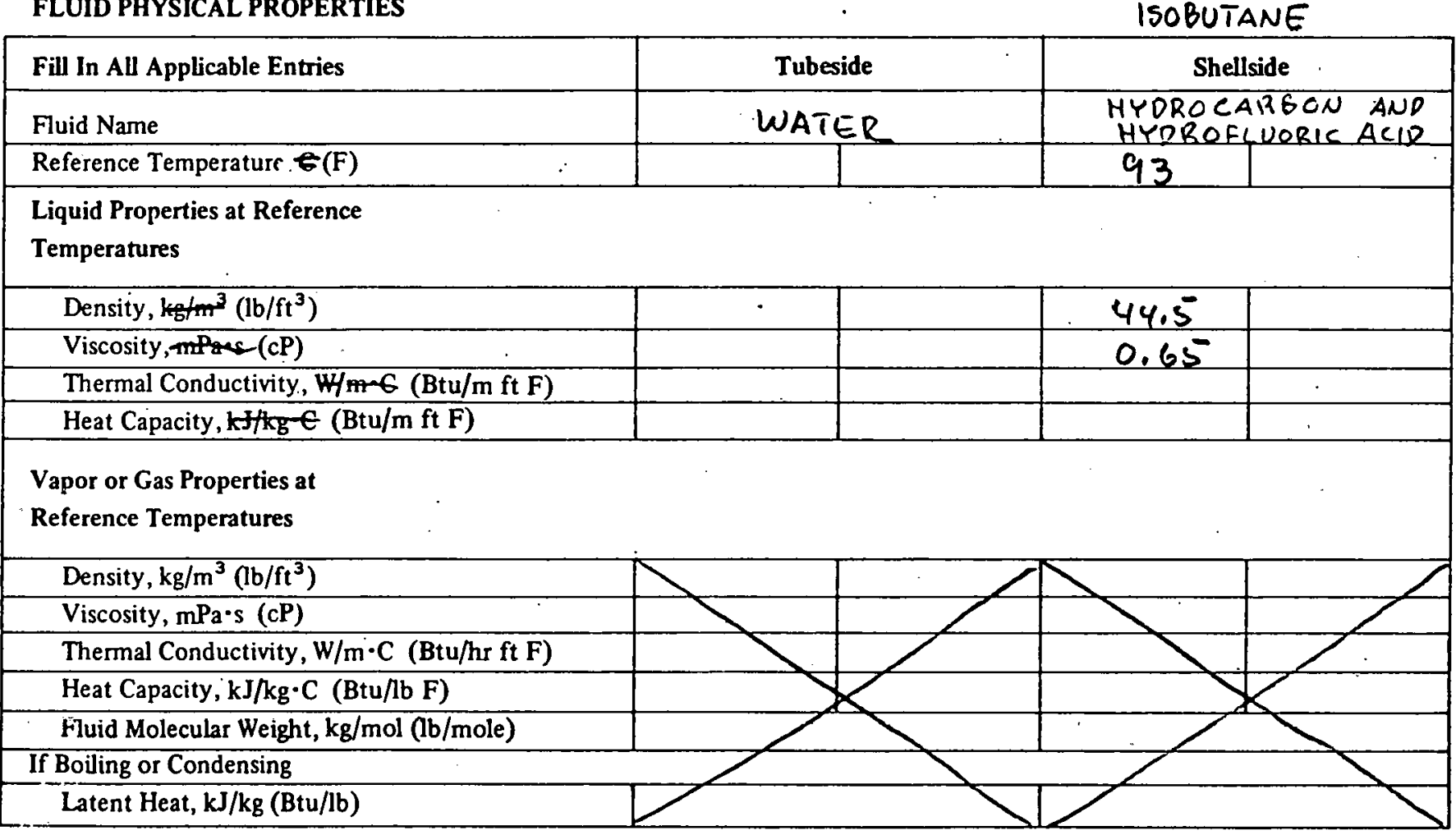




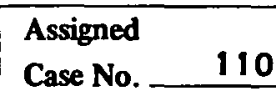

\section{VIBRATION AND DAMAGE DESCRIPTION}

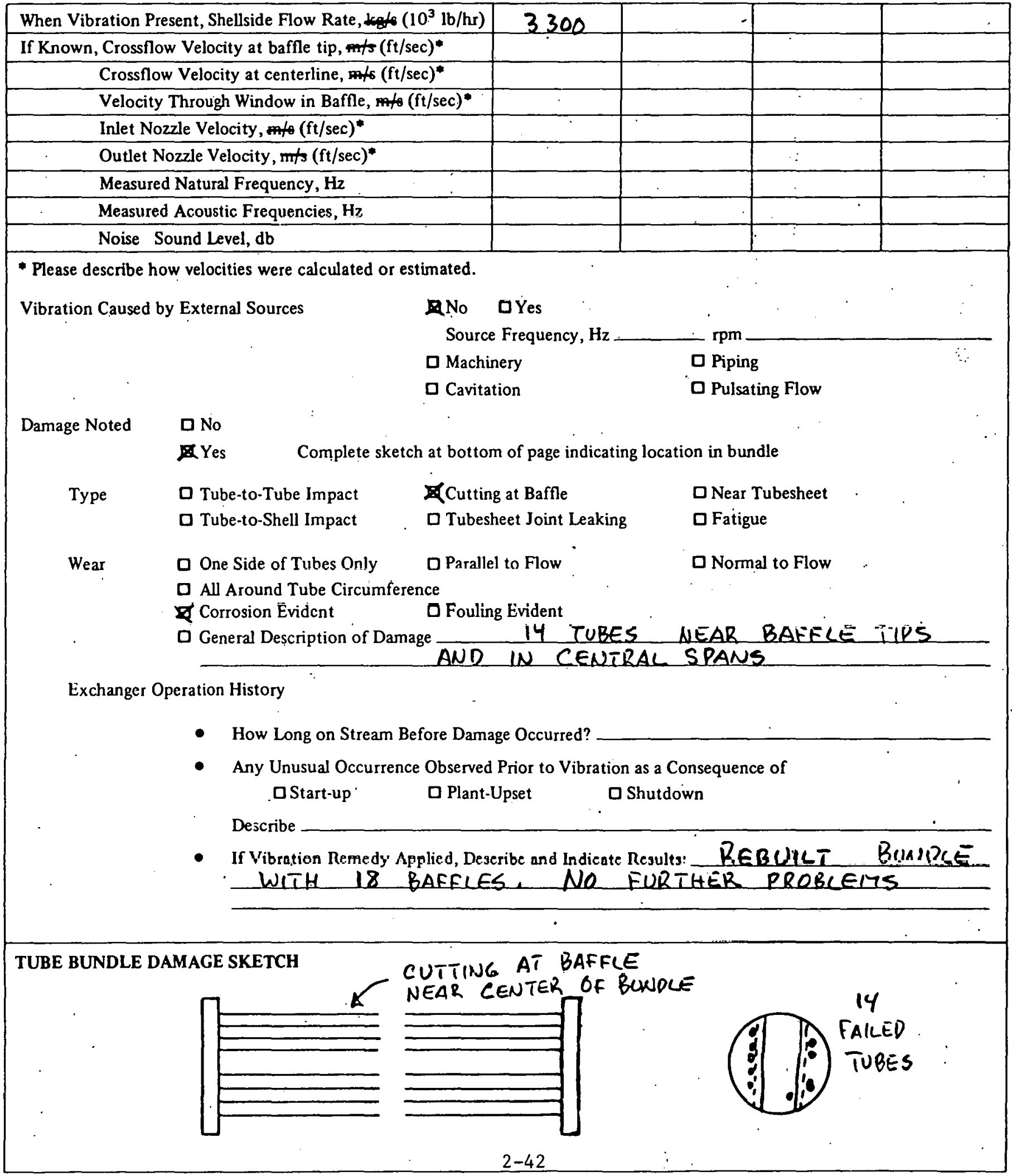


To protect the identity of the organization submitting this case, HTRI has assigned a case number. Additionally the data on pages 2, 3, and 4 have been reviewed to ensure that they do not include any propietary information.

Assigned

Case No.
111

This is a replacement for the original page 1 that provides space for additional comments, drawings, photographs, etc.

\section{Sưmmary}

This 45-in. diameter by 40-ft long CEM TEMA style heat exchanger is the same shell as for Case 110 with a replacement bundle. These are 18 double-segmental baffles instead of the oxiginal 12: The service and the fluids are the same. This exchanger resulted in a higher shellside pressure drop; however, added pump capacity was available. The exchanger has operated for several years without any vibration problems.

The information is limited to that included on the data form. 


\begin{tabular}{|c|c|c|c|}
\hline Type & $\begin{array}{l}\text { Q TEMA Exchanger } \\
\square \text { Special Exchanger }\end{array}$ & $\begin{array}{l}\text { Designation } \subset \in N \\
\text { (Deșcribe) }\end{array}$ & $\begin{array}{ll}\text { Shell Orientation } & \text { Horizontal } \\
& \square \text { Vertical }\end{array}$ \\
\hline
\end{tabular}

SHELL GEOMETRY (Complete Sketch Below)

Inside Diameter, (in.) 45

Wall Thickness, (in.) ___ Material

Inlet Nozzle ID, $\mathrm{mm}$ (in.) _ $\quad 24$

Outlet Nozzle ID, (in.) 24

Impingement Protection \&No $\square$.Yes

(Describe).

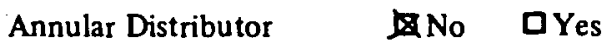

Open Cut Area $\operatorname{mm}^{2}$ (in. ${ }^{2}$ )

Nozzle-to-First Tube Row Distance, (in.) 1.5

\section{CROSS BAFFLE GEOMETRY}

Type a Segmental; Double-Segmental D Disc/Doughnut

$\square$ Triple-Segmental; $\square$ No-Tubes-in-Window

Baffle Cut, \% Shell Diameter $\quad 26.5$

Cut Orientation Relative to Axis of Inlet Nozzle

Inlet Baffle $\square$ Perpendicular Parallel $\square 45^{\circ}$

Central Baffles $\square$ Perpendicular $\otimes$ Parallel $\square 45^{\circ}$

Baffle Thickness, mm (in.) 0.625 Material CS

Diametral Clearances Shell-to-baffle, (in.) 0.25

Tube-to-baffle mm (in.) 0.0156

Bundle-to-shell, (in.) 1.125

Number of Baffles Along Length of Shell 18

Baffle Spacing, (in.)___entral 25

Inlet 24.13 outlet 24.13

Unsupported Tube Span Lengths, (in.)

Longest 50 Inlet 49.13 Outlet 49.13

\section{TUBE GEOMETRY}

Outside Diameter, $-m m$ (in.)

Wall Thickness, (in.) 0.134 Material

Tube Lengths

Straight Tube, Inside Tubesheets, (in.) 473.25

U.Tube, Tubesheet to Bend Tangent, mm (in.) _ _

Tube Pitch, $\operatorname{man}$ (in.) 1.25

Layout (Please Circle)

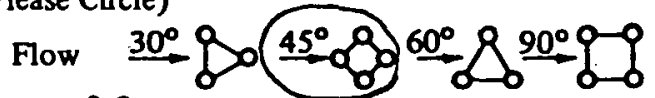

No. of Tubes 930 No. of Tubepasse 1.

Füst Tubepass Countercurrent $\square$ Cocurrent

Tube-to-Tubesheet Joint

FWelded Roller Expanded $\square$ Other

If U-Tube

Maximum Bend Radius, mm(in.)

Bend Orientation Relative to Axis of Shellside Inlet Nozzle

$\square$ Perpendicular $\square$ Parallel -

If Bend Supported, Describe in Comments Below

If Finned Tubes

Finoton (Fins/in.) __ Fin Material _-

Diameter, $\operatorname{mm}($ in.), Root __ Over Fins _ _

If Enhanced Surface Tubes

(Describe)

\section{DE'TUNING BAFFLE}

If Detuning Baffle Used to Control Acoustic

Vibration, Indicate Position on Sketch Below

\section{COMMENTS AND SKETCH}

Complete sketches by drawing in tubeside and shellside nozzles.

indicate inlet nozzles with an arrow.

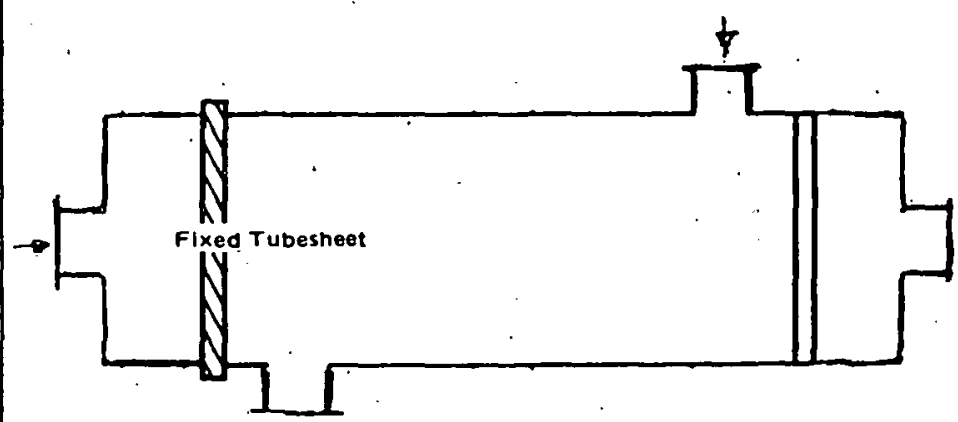

Show shellside inlet nozzle location, baffle cut orlentation, and impingement devices.

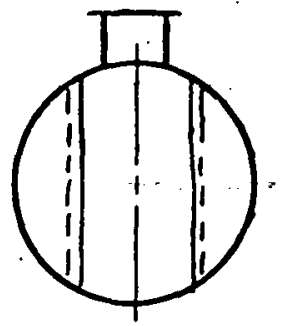
政 


\begin{tabular}{|c|c|c|c|c|c|}
\hline & & \multicolumn{2}{|c|}{ Reference Condition A } & \multicolumn{2}{|c|}{ Reference Condition B } \\
\hline & & Tubeside & Shellside & Tubeside & Shellside \\
\hline Flow Rate, kgts $\left(10^{3} \mathrm{lb} / \mathrm{hr}\right)$ & & & 3300 & & \\
\hline Inlet Temperature, $\mathcal{e ( F )}$ & & & 95 & & \\
\hline Outlet Temperature, $\in(F)$ & & & 90 & & \\
\hline Inlet Pressure, $k P^{2}$ (psia) Absolute & $\vdots$ & & 200 & & \\
\hline Measured $\Delta \mathrm{P}, \mathrm{PP}_{\mathrm{Q}}(\mathrm{psi})$ & & & - & & \\
\hline Inlet Weight Fraction Vapor & & 0 & 0 & & \\
\hline Outlet Weight Fraction Vapor & & 0 & 0 & & \\
\hline Vibration Observed & & $8 \mathrm{No}$ & & DNo & DYes \\
\hline
\end{tabular}

\section{FLUID PHYSICAL PROPERTIES}

ISOBUTANE

\begin{tabular}{|c|c|c|}
\hline Fill In All Applicable Entries & Tubeside & Shellside \\
\hline Fluid Name & WATER & $\begin{array}{l}\text { HYOROCARBOU PLUS } \\
\text { HYOROFUVORIS ACIC }\end{array}$ \\
\hline Reference Temperature (F) & & 93 \\
\hline \multicolumn{3}{|l|}{ Liquid Properties at Reference } \\
\hline \multicolumn{3}{|l|}{ Temperatures } \\
\hline Density, $\mathrm{kgtm}^{3}\left(\mathrm{lb} / \mathrm{ft}^{3}\right)$ & & 44.5 \\
\hline Viscosity, mPas (cP) & & 0.65 \\
\hline \multicolumn{3}{|l|}{ Thermal Conductivity, $\mathrm{W} / \mathrm{m}-\mathrm{G}(\mathrm{Btu} / \mathrm{m} \mathrm{ft} \mathrm{F})$} \\
\hline Heat Capacity, kJ/kg $\in(B t u / m$ ft F) & & \\
\hline \multirow{2}{*}{\multicolumn{3}{|c|}{$\begin{array}{l}\text { Vapor or Gas Properties at } \\
\text { Reference Temperatures }\end{array}$}} \\
\hline & & \\
\hline \multicolumn{3}{|l|}{ Density, $\mathrm{kg} / \mathrm{m}^{3}\left(\mathrm{lb} / \mathrm{ft}^{3}\right)$} \\
\hline \multicolumn{3}{|l|}{ Viscosity, $\mathrm{mPa} \cdot \mathrm{s}(\mathrm{cP})$} \\
\hline \multicolumn{3}{|l|}{ Thermal Conductivity, $\mathrm{W} / \mathrm{m} \cdot \mathrm{C}(\mathrm{Btu} / \mathrm{hr} \mathrm{ft} \mathrm{F})$} \\
\hline \multicolumn{3}{|l|}{ Heat Capacity, $\mathrm{kJ} / \mathrm{kg} \cdot \mathrm{C}(\mathrm{Btu} / \mathrm{lb} \mathrm{F})$} \\
\hline \multicolumn{3}{|l|}{ Fluid Molecular Weight, $\mathrm{kg} / \mathrm{mol}$ (lb/mole) } \\
\hline \multicolumn{3}{|l|}{ If Boiling or Condensing } \\
\hline Latent Heat, $\mathrm{kJ} / \mathrm{kg}(\mathrm{Btu} / \mathrm{lb})$ & & \\
\hline
\end{tabular}




\section{VIBRATION AND DAMAGE DESCRIPTION}

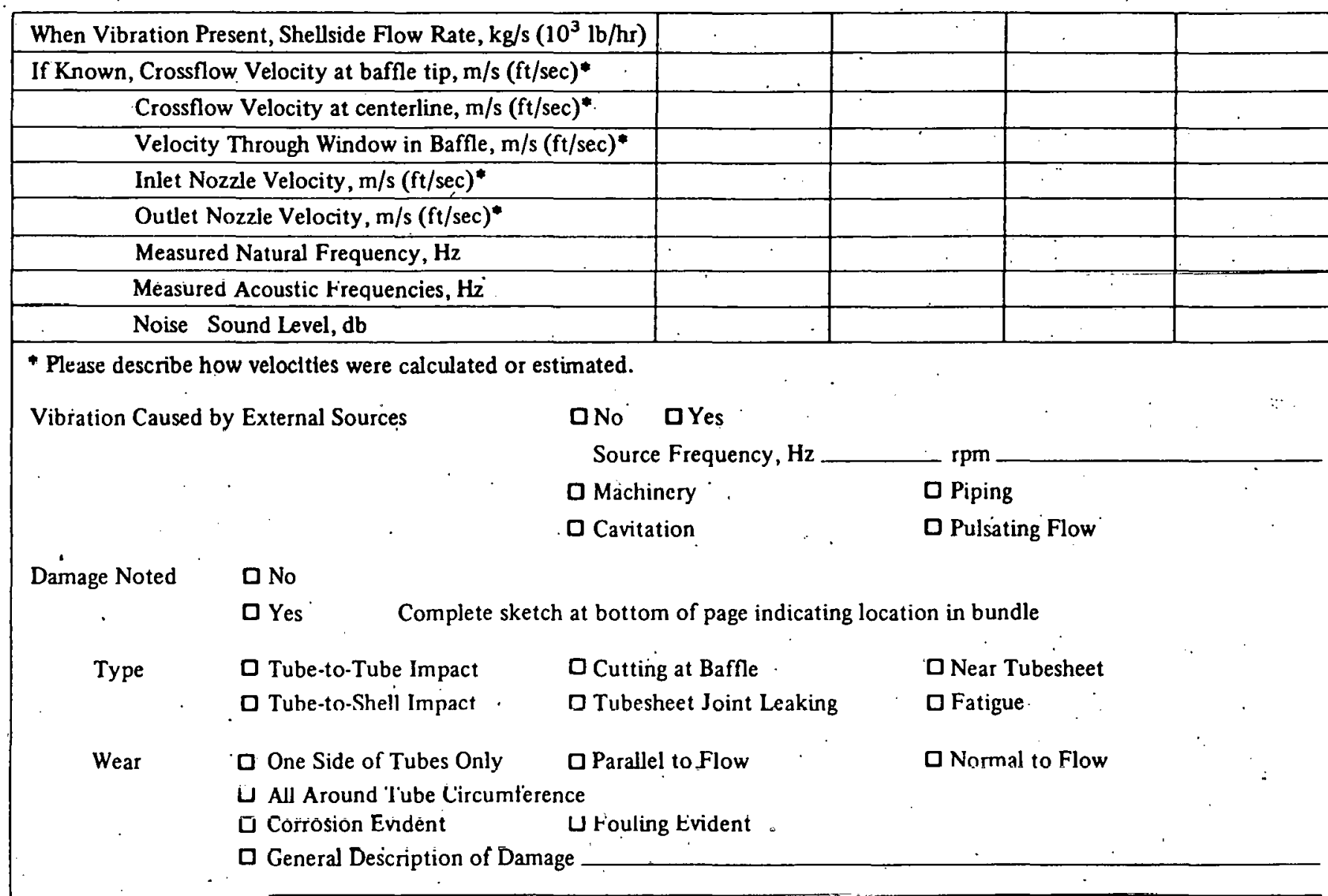

Exchanger Operation History

- How Long on Stream Before Damage Occurred?

- Any Unusual Occurrence Observed Prior to Vibration as a Consequence of
DStart-up
$\triangle$ Plant-Upset
Q Shutdown

Describe

- If Vibration Remody Appliod, Deneribe and Lidicate Results:

\section{TUBE BUNDLE DAMAGE SKETCH}
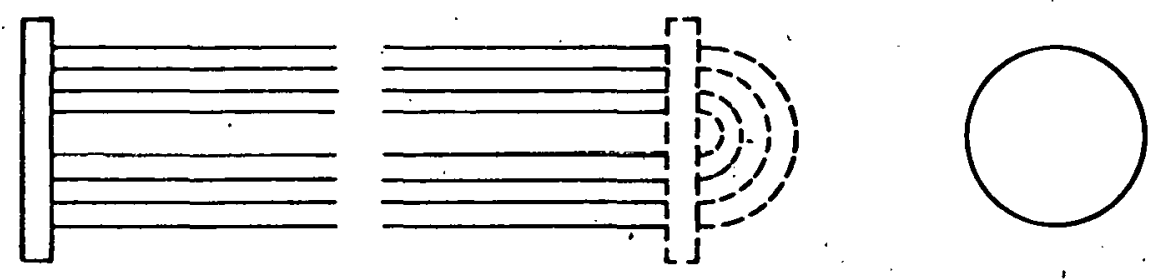

$2-46$ 
To protect the identity of the organization submitting this case, HTRI has assigned a case number. Additionally the data on pages 2, 3, and 4 have been reviewed to ensure that they do not include any propietary information.

Assigned

Case No.

This is a replacement for the original page 1 that provides space for additional comments, drawings, photographs, etc.

Summary

This 43-in. diameter by 19-ft long AJS TEMA style heat exchanger has an annular distribution at the center inlet with a series of slots to direct half of the shellside flow of air to each of the two ends of the exchanger. Cooling tower water flowed in the tube side. The bundle has segmental baffles with relatively short unsupported span lengths for $3 / 4-i n$. admiralty tubes. The tube field is arranged in an inline layout with a pitch-todiameter ratio of 1.33 . The indication is that this exchanger had "frequent tube leaks." Unfortunately, there is no indication as to where the leaks occurred. Tube vibration is given as the probable cause.

For this case, there are partial construction drawings, a field inspection report, and the exchanger specification sheet. 


\begin{tabular}{|c|c|c|c|c|}
\hline Type & $\begin{array}{l}\text { R TEMA Exchanger } \\
\text { O Special Exchanger }\end{array}$ & $\begin{array}{l}\text { Designation AJS } \\
\text { (Describe) }\end{array}$ & Shell Orientation & Q Horizontal \\
\hline
\end{tabular}

SHELL GEOMETRY (Complete Sketch Below)

\begin{tabular}{|c|}
\hline Inside Diameter, (in.) $\quad 43$ \\
\hline Wall Thickness, (in.) ___ Material \\
\hline Inlet Nozzle ID, (in.) $\quad 16$ \\
\hline Outlet Nozzle ID, (in.) 12 \\
\hline $\begin{array}{l}\text { Impingement Protection No } \quad \text { Nos } \\
\text { (Describe) }\end{array}$ \\
\hline $\begin{array}{l}\text { Annular Distributor } \quad \text { No }{ }^{2} \text { (ines } \\
\text { Open Cut Area } \\
\end{array}$ \\
\hline Nozzle-to-First Tube Row Distance, (in.) 1.625 \\
\hline CROSS BAFFLE GEOMETRY \\
\hline $\begin{array}{l}\text { Type Segmental; } \square \text { Double-Segmental } \square \text { Disc/Doughnut } \\
\square \text { Triple-Segmental; } \square \text { No-Tubes-in-Window } \\
\text { Baffle Cut, \% Shell Diameter } \quad 33\end{array}$ \\
\hline Cut Orientation Relative to Axis of Inlet Nozzle \\
\hline $\begin{array}{l}\text { Inlet Baffe } \square \text { Perpendicular Parallel } \square 45^{\circ} \\
\text { Central Baffles } \square \text { Perpendicular Parallel } \square 45^{\circ}\end{array}$ \\
\hline Baffle Thickness, (in.) 0.5 Material $C S$ \\
\hline $\begin{array}{r}\text { Diametral Clearances Shell-to-baffle, (in.) } \frac{0.25}{0.0156} \\
\qquad \begin{array}{r}\text { Tube-to-baffle (in.) } 0.25 \\
\text { Bundle-to-shell, }\end{array}\end{array}$ \\
\hline Number of Baffles Along Length of Shell $8+$ VIVIDER \\
\hline 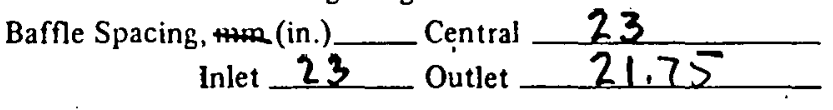 \\
\hline $\begin{array}{l}\text { Unsupported Tube Span Lengths, } \\
\text { Longest } 46 \text { Inlet } 46 \text { Outlet } 44.75\end{array}$ \\
\hline
\end{tabular}

\section{TUBE GEOMETRY}

Outside Diameter, mm-(in.) $\quad 0.750$

Wall Thickness, mm (in.) 0.065 Material AQMIRALTY Tube Lengths

Straight Tube, Inside Tubesheets, (in.) 227.5

U-Tube, Tubesheet to Bend Tangent, min-(in.)

Tube Pitch, (in.) 1.00 .

Layout (Please Circle)

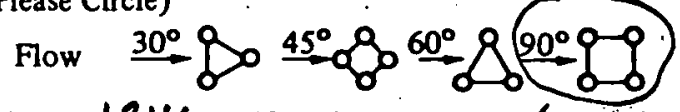

No. of Tubes 1214 No. of Tubepasses 6

First Tubepass 1 Countercurrent

Tube-to-Tubesheet Joint

$\square$ Welded Roller Expanded $\square$ Other

If U.Tube

Maximum Bend Radius, (in.) _ -

Bend Orientation Relative to Axis of Shellside Inlet Nozzle

口 Perpendicular O Parallel

If Bend Supported, Describe in Comments Below

If Finned Tubes

(Fins/in.) _ — Fin Material _-

Diameter; (in.), Root - Over Fins -

If Enhanced Surface Tubes _ _

(Describe)

DEIUNING BAFFLE

If Detuning Baffle Used to Control Acoustic

Vibration, Indicate Position on Sketch Below

\section{COMMENTS AND SKETCH}

Complete sketches by drawing in tubeside and shellside nozzles. Indicate inlet nozzles with an arrow.

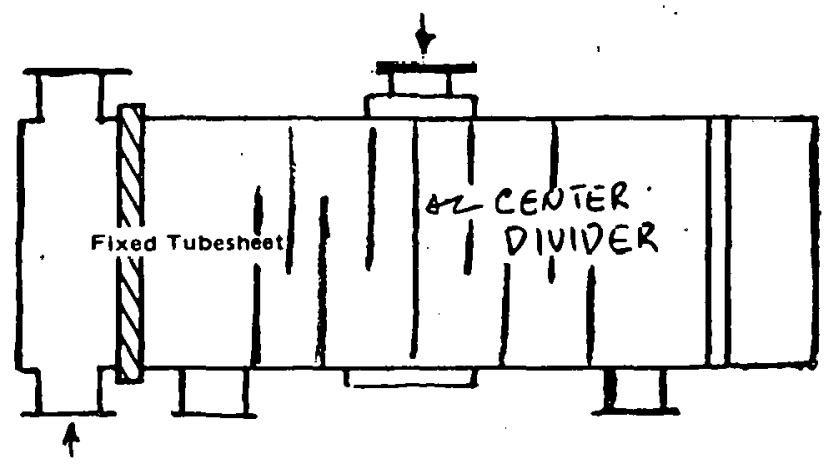

Show shellside Inlet nozzle location, baffle cut orientation, and Impingement devices.

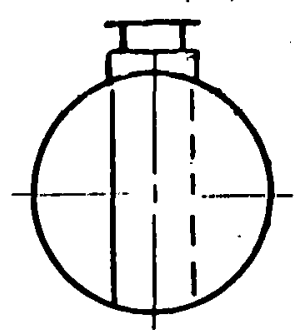




\begin{tabular}{l} 
Assigned \\
Case No. 112 \\
\hline
\end{tabular}

\section{PROCESS CONDITIONS}

\begin{tabular}{|c|c|c|c|c|}
\hline - & \multicolumn{2}{|c|}{ Reference Condition A } & \multicolumn{2}{|c|}{ Reference Condition B } \\
\hline Flow Rate, $\mathrm{k}_{\mathrm{b}} / \mathrm{f}\left(10^{3} \mathrm{lb} / \mathrm{hr}\right)$ & 378 & 168 & & \\
\hline Inlet Temperature, $\ell(F)$ & 90 & 225 & & \\
\hline Outlet Temperature, $\in(F)$ & 105 & 105 & & \\
\hline Inlet Pressure, $\mathrm{kPa}$ (psia) Absolute & 65 & 118 & & \\
\hline Inlet Weight Fraction Vapor & 0 & 1.0 & & \\
\hline Outlet Weight Fraction Vapor & 0 & 1.0 & & \\
\hline Vibration Observed & ONo & & DNo & \\
\hline
\end{tabular}

\section{FLUID PHYSICAL PROPERTIES}

\begin{tabular}{|c|c|c|}
\hline Fill In All Applicable Entries & Tubeside & Shellside \\
\hline Fluid Name & WATER & AIR \\
\hline \multicolumn{3}{|l|}{ Reference Temperaturc $\in(F)$} \\
\hline \multicolumn{3}{|l|}{$\begin{array}{l}\text { Liquid Properties at Reference } \\
\text { Temperatures }\end{array}$} \\
\hline \multicolumn{3}{|l|}{ Density, $\mathrm{kg} / \mathrm{m}^{3}\left(\mathrm{lb} / \mathrm{ft}^{3}\right)$} \\
\hline \multicolumn{3}{|l|}{ Viscosity, $\mathrm{mPa} \cdot \mathrm{s}(\mathrm{cP})$} \\
\hline \multicolumn{3}{|l|}{ Thermal Conductivity, $\mathrm{W} / \mathrm{m} \cdot \mathrm{C}(\mathrm{Btu} / \mathrm{m} \mathrm{ft} \mathrm{F})$} \\
\hline \multicolumn{3}{|l|}{ Heat Capacity, $\mathrm{kJ} / \mathrm{kg} \cdot \mathrm{C}(\mathrm{Btu} / \mathrm{m} \mathrm{ft} \mathrm{F})$} \\
\hline \multicolumn{3}{|l|}{ Vapor or Gas Properties at } \\
\hline \multicolumn{3}{|l|}{ Density, $\mathrm{kg} / \mathrm{m}^{3}\left(\mathrm{lb} / \mathrm{ft}^{3}\right)$} \\
\hline \multicolumn{3}{|l|}{ Thermal Conductivity, $\mathrm{W} / \mathrm{m} \cdot \mathrm{C}(\mathrm{Btu} / \mathrm{hr} \mathrm{ft} \mathrm{F})$} \\
\hline \multicolumn{3}{|l|}{ Heat Capacity, $\mathrm{kJ} / \mathrm{kg} \cdot \mathrm{C}(\mathrm{Btu} / \mathrm{lb} \mathrm{F})$} \\
\hline Fluid Molecular Weight, $\mathrm{kg} / \mathrm{mol} \mathrm{(} \mathrm{lb} / \mathrm{mole})$ & & . \\
\hline \multicolumn{3}{|l|}{ If Boiling or Condensing } \\
\hline Latent Heat $; \mathrm{kJ} / \mathrm{kg}(\mathrm{Btu} / \mathrm{lb})$ & & $\longrightarrow$ \\
\hline
\end{tabular}




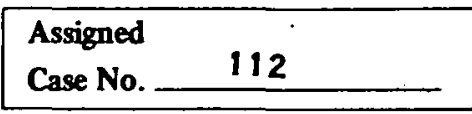

\section{VIBRATION AND DAMAGE DESCRIPTION}

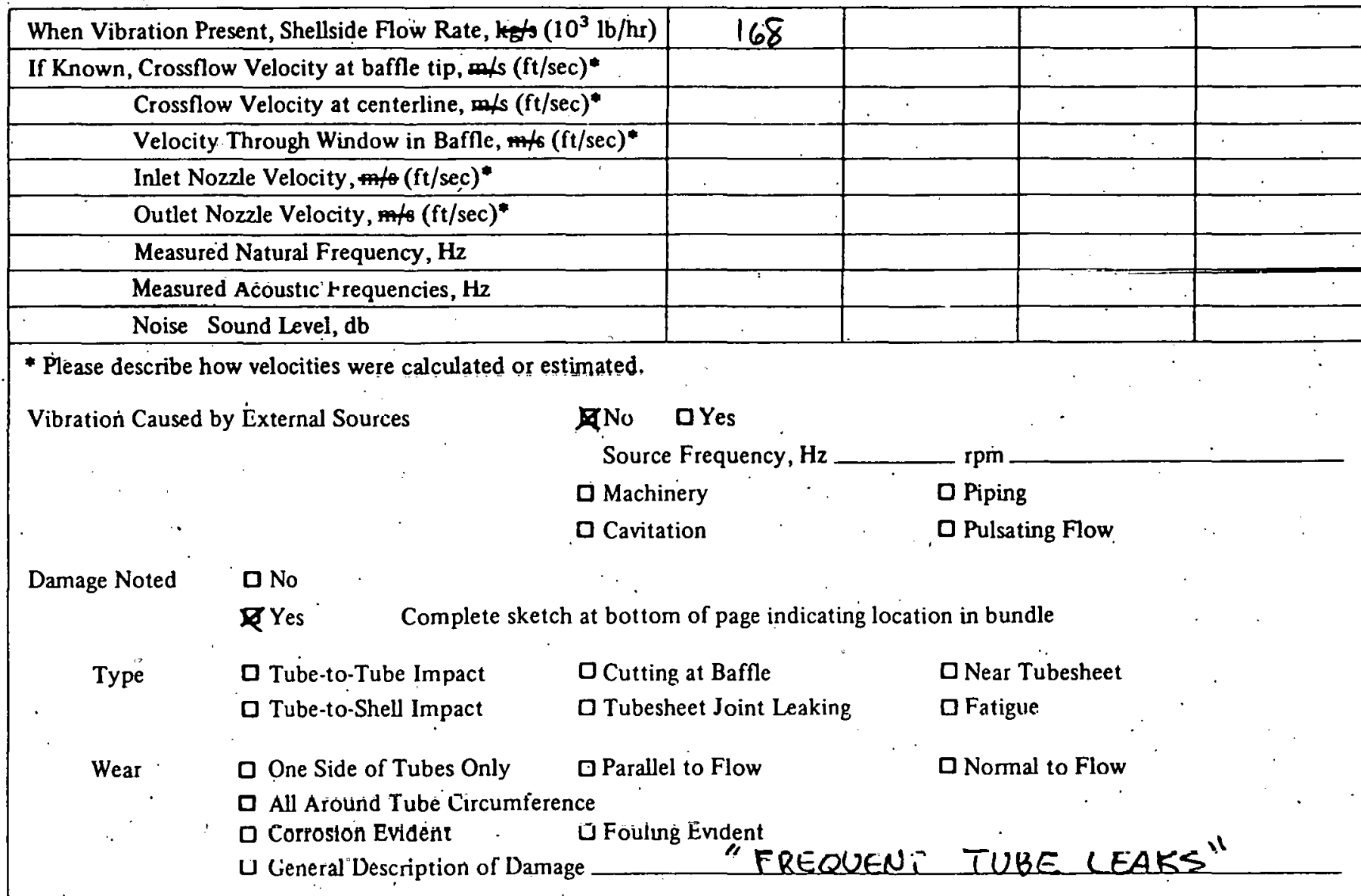

Exchanger Operation History

- How Long on Stream Before Damage Occurred?

- Any Unusual Occurrence Observed Prior.to Vibration as a Consequence of
D Start-up
口 Plant-Upset
口 Shutdown

Describe

- If Vibration Remedy Applied. Describe and Indicatc. Resiılts:

\section{TUBE BUNDLE DAMAGE SKETCH}
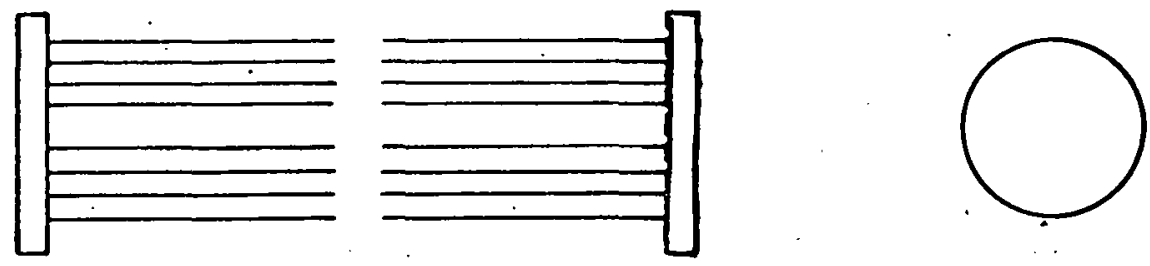

$2-50$ 
To protect the identity of the organization submitting this case, HTRI has assigned a case number. Additionally the data on pages 2, 3, and 4 have been reviewed to ensure that they do not include any propietary information.

This is a replacement for the original page 1 that provides space for

Assigned

Case No.

\section{Summary}

This 17-in. diameter by 16-ft long AEU TEMA style heat exchanger has cooling water flowing inside the two tube passes formed by the U-tubes. On the shell side a hydrocarbon designated "Atmospheric Top Pumparound" flows through a bundle with segmental baffles with very little overlap so the flow is mostly parallel to the tubes: Originally admiralty tubes were used and these suffered damage. The inspection report indicates evidence of "fretting corrosion with numerous penetrations under baffles." The indication is that there was a combined effect of vibration and corrosion. The tubes that failed were near the longitudinal baffle passlane and at the baffle plates. The cutting action resulted in the tubes being worn through on one side, but the specific orientation is not noted.

For this case, there are in addition to the data form, an inspection report, transmittal letter, and an indication of the remedial action taken. The bundle was retubed with 430 alloy stainless steel except for the bottom row of fin tubes which were with titanium. No further problems have been indicated. 


\begin{tabular}{l} 
Assigned \\
Case No. 113 \\
\hline
\end{tabular}

\begin{tabular}{|c|l|c|}
\hline Type $\begin{array}{l}\text { KeMA Exchanger } \\
\square \text { Special Exchanger }\end{array}$ & $\begin{array}{l}\text { Designation_AEU } \\
\text { (Describe) }\end{array}$ & Shell Orientation $\begin{array}{l}\text { Horizontal } \\
\text { QVertical }\end{array}$ \\
\hline
\end{tabular}

\section{SHELL GEOMETRY (Complete Sketch Below)}

Inside Diameter, (in.) 17.25

Wall Thickness, (in.) 0.375 Material_CS

Inlet Nozzle ID, (in.) 7.625

Outlet Nozzle ID, (in.) $7.625^{-}$

Impingement Protection No DYes

(Describe)

Annular Distributor No DYes

Open Cut Area $\mathrm{mm}^{2}$ (in. $^{2}$ )

Nozzle-to-First Tube Row Distance, (in.) 3.4375

\section{CROSS BAFFLE GEOMETRY}

Type \Segmental; 口 Double-Segmental $\square$ Disc/Doughnut

$\square$ Triple-Segmental; $\square$ No-Tubes-in-Window

Baffle Cut, \% Shell Diameter 43.5

Cut Orientation Relative to Axis of Inlet Nozzle

Inlet Baffle $\square$ Perpendicular \& Parallel $045^{\circ}$

Central Baffles $\square$ Perpendicular Parallel $045^{\circ}$

Baffle Thickness, (in.) 0.375 Material CS

Diametral Clearances Shell-to-baffle, (in.) 0,123

Tube-to-baffle (in.) 0,0156

Bundle-to-shell, (in.) .2 .0

Number of Baffles Along Length of Shell $\_6$

Baffle Spacing, (in.)__ Central 27

$$
\text { Inlet } 27 \text { Outlet } 27
$$

Unsupported Tube Span Lengths, (in.)

Longest 54 . Inlet 54 Outlet 54

\section{TUBE GEOMETRY}

Outside Diameter, mm (in.) 7.50

Wall Thickness, (in.) 0.065 Material ARMB ALIY

Tube Lengths

BRASS

Straight Tube, Inside Tubesheets, (in.) _ _ -

U-Tube, Tubesheet to Bend Tangent, (in.) 189

Tube Pitch, $\rightarrow$ m (in.) $\quad 1,00$

Layout (Please Circle)

Flow $\quad 30^{\circ}$ O

No. of Tubes_ 116

No. of Tubepasses

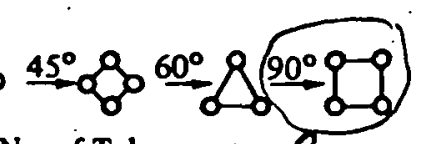

First Tubepass DCountercurrent DCocurrent?

Tube-to-Tubesheet Joint

$\square$ Welded Roller Expanded 0 Other

If U-Tube

Maximum Bend Radius, (in.) 7.25

Bend Orientation Relative to Axis of Shellside Inlet Nuzale

D Perpendicular Parallel

If Bend Supported, Describe in Comments Below

If Finned Tubes

Find (Fins/in.) ___ Fin Material

Diameter, (in.), Root — Over Fins

If Enhanced Surface Tubes

(Describe)

DETUNING BAFTLE

If Detuning Baffle Used to Control Acoustic

Vibration, Indicate Position on Sketch Below

\section{COMMENTS AND SKETCH}

Complete sketches by drawing in tubeside and shellside nozzles.

indicate Inlet nozzles with an arrow.

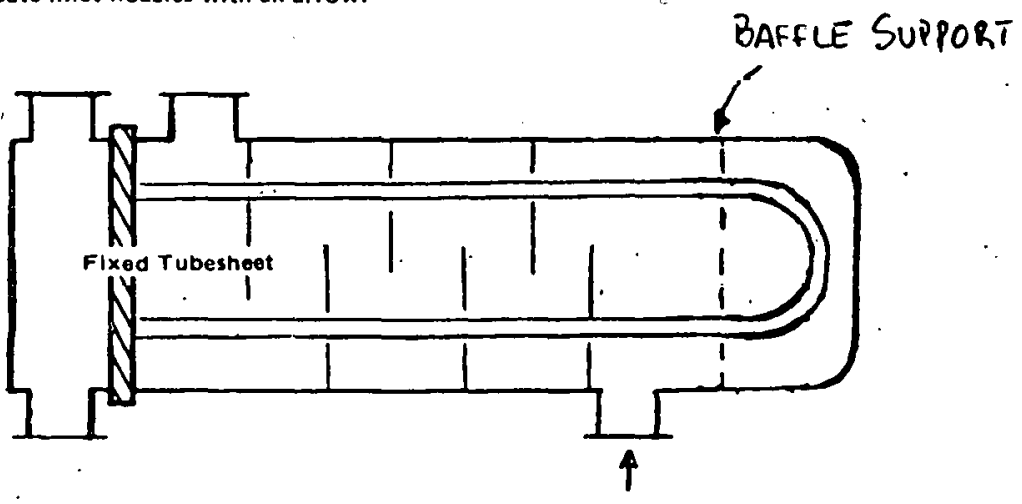

Show shellside Inlet nozzle location, baffle cut orientation, and impingement devices.

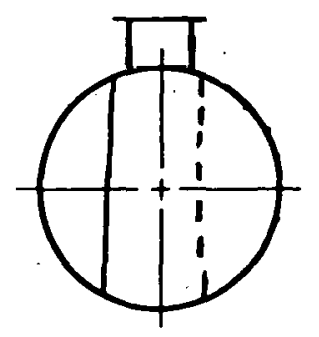




\section{PROCESS CONDITIONS}

\begin{tabular}{|c|c|c|c|c|}
\hline & \multicolumn{2}{|c|}{ Reference Condition $\mathrm{A}$} & \multicolumn{2}{|c|}{ Reference Condition B } \\
\hline Flow Rate, $\mathrm{kg} / \mathrm{s}\left(10^{3} \mathrm{lb} / \mathrm{hr}\right)$ & & 372.4 & & \\
\hline Inlet Temperature, $\in(F)$ & 70 & 234 & & \\
\hline Outlet Temperature, $\in(F)$ & 100 & 176 & & \\
\hline \multicolumn{5}{|c|}{ Inlet Pressure, kPo-(psia) Absolute } \\
\hline \multicolumn{5}{|c|}{ Measured $\Delta P, k_{2}$ (psi) } \\
\hline Inlet Weight Fraction Vapor & 0 & 0 & & $\therefore$ \\
\hline Outlet Weight Fraction Vapor & 0 & 0 & & \\
\hline Vibration Observed & \multicolumn{2}{|c|}{ DNo EYes } & \multicolumn{2}{|c|}{ QYes } \\
\hline
\end{tabular}

\section{FLUID PHYSICAL PROPERTIES}

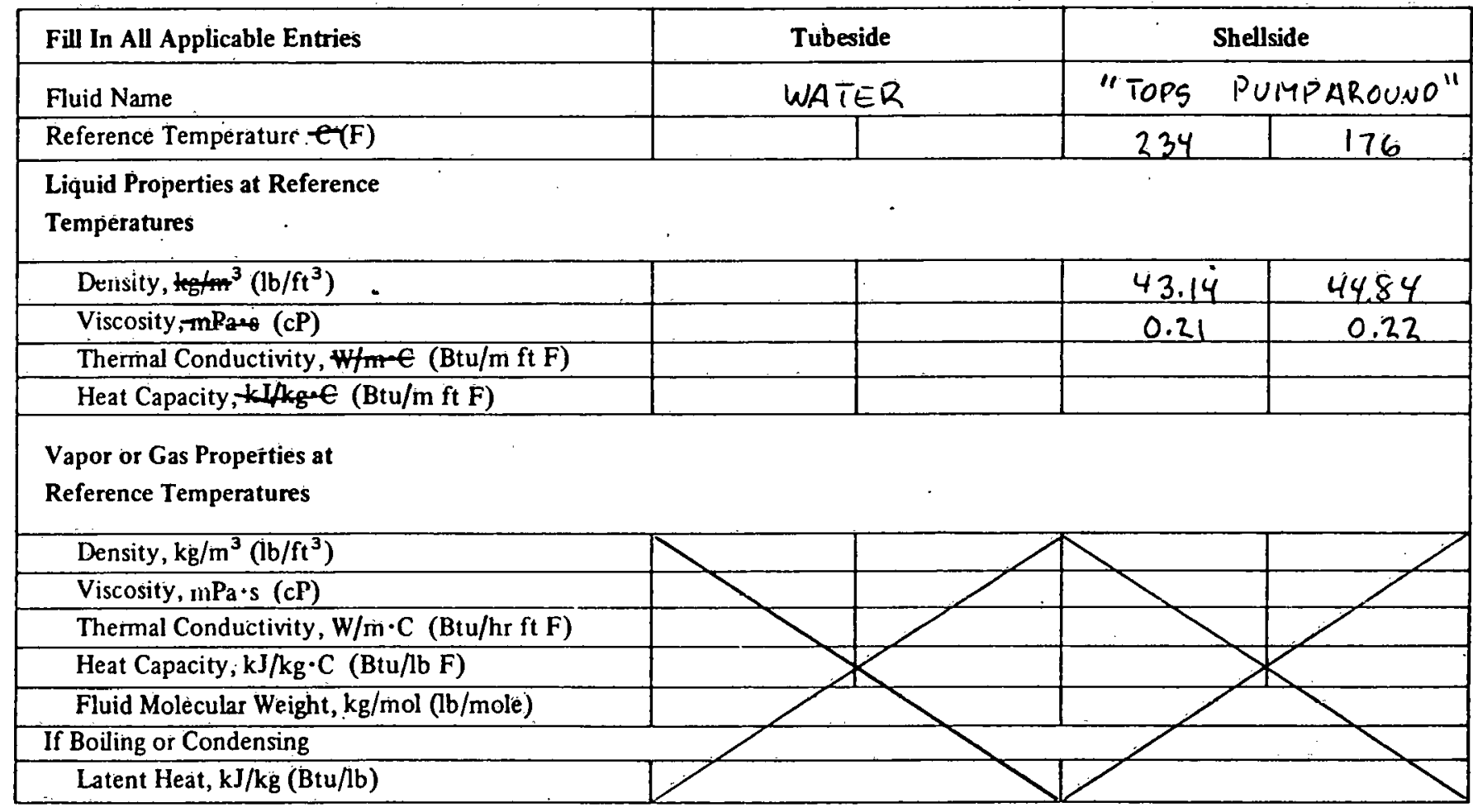


VIBRATION AND DAMAGE DESCRIPTION

\begin{tabular}{|c|}
\hline When Vibration Present, Shellside Flow Rate, $\left(10^{3} \mathrm{lb} / \mathrm{hr}\right)$ \\
\hline If Known, Crossflow Velocity at baffle tip, $(\mathrm{ft} / \mathrm{sec})^{*}$ \\
\hline Crossflow Velocity at centerline, $(\mathrm{ft} / \mathrm{sec})^{*}$ \\
\hline Velocity Through Window in Baffle, $/ \mathrm{ft} / \mathrm{sec})^{*}$ \\
\hline Inlet Nozzle Velocity, $\mathrm{ft} / \mathrm{sec})^{*}$ \\
\hline Outlet Nozzle Velocity, $(\mathrm{ft} / \mathrm{sec})^{*}$ \\
\hline Measured Natural Frequency, $\mathrm{Hz}$ \\
\hline Measured Acoustic Frequencies, $\mathrm{Hz}$ \\
\hline Noise Sound Level, db \\
\hline
\end{tabular}

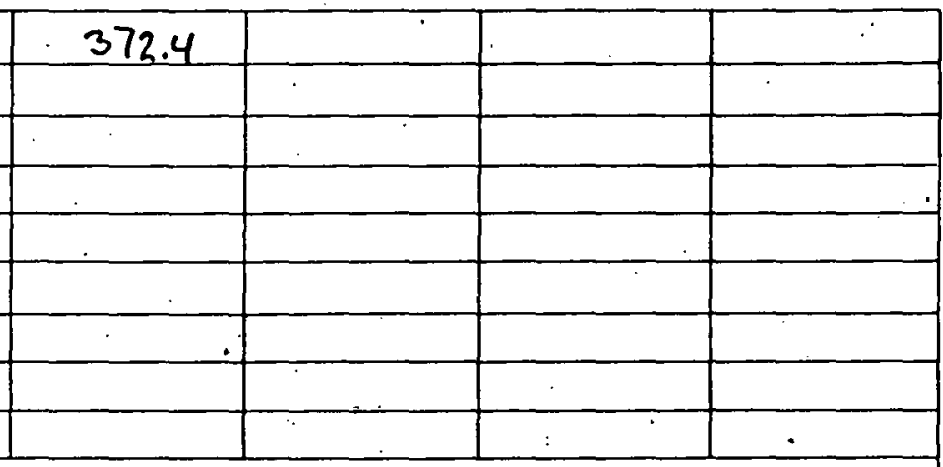

* Please describe how velocities were calculated or estimated.

Vibration Caused by External Sources

Damage Noted

D No

XYes
ENo DYes

Source Frequency, $\mathrm{Hz}$

Dachinery

Q Cavitation rpm

$\square$ Piping

D Pulsating Flow

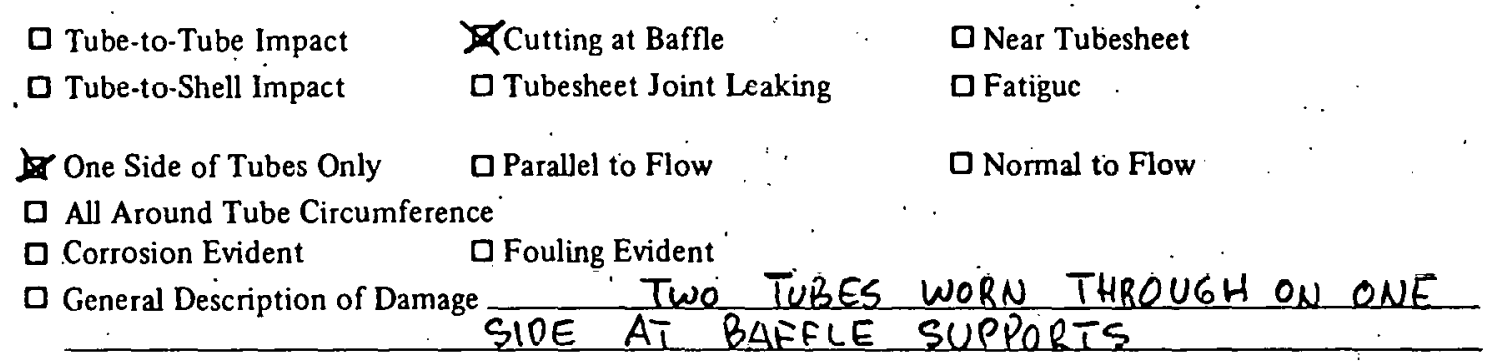

Exchanger Operation History

- How Long on Stream Before Damage Occurred?

- Any Unusual Occurrence Observed Prior to Vibration as a Consequence of
口Start-up
D Plant-Upset
D Shutdown

Describe

- If Vibraliun Remedy Applied, Desuibe aud Ludiuale Results.

\section{TUBE BUNDLE DAMAGE SKETCH}
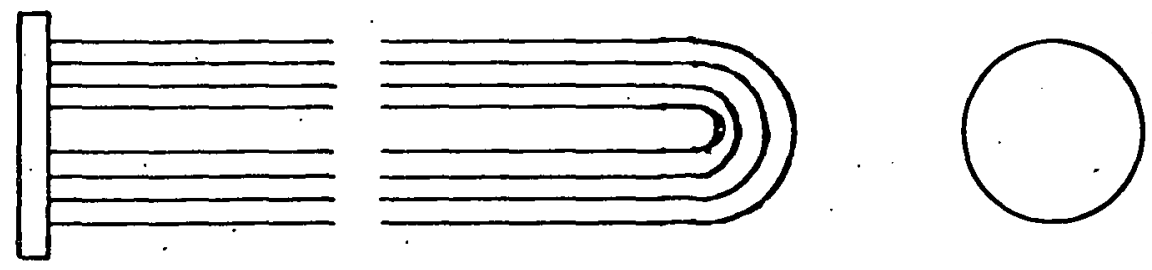
To protect the identity of the organization submitting this case, HTRI has assigned a case number. Additionally the data on pages 2,3, and 4 have been reviewed to ensure that they do not include any propietary information.

Assigned

Case No.

This is a replacement for the original page 1 that provides space for additional comments, drawings, photographs, etc.

\section{Summary}

This 56-in. diameter by 50-ft long AEL TEMA style heat exchanger has a hydrocarbon gas stream on both the shell side and the tube side. The "lean gas" is heated on the shell side where the bundle has 19 "double double-segmental" baffles. See sketch below. The "rich gas" is cooled on the tube side in a single tube pass. There was no impingement device under the shellside inlet nozzle. Tubes failed in "top three rows under shell inlet nozzle."

For this case, in addition to the data form is a description of the process conditions and geometry including the sketch reproduced below.

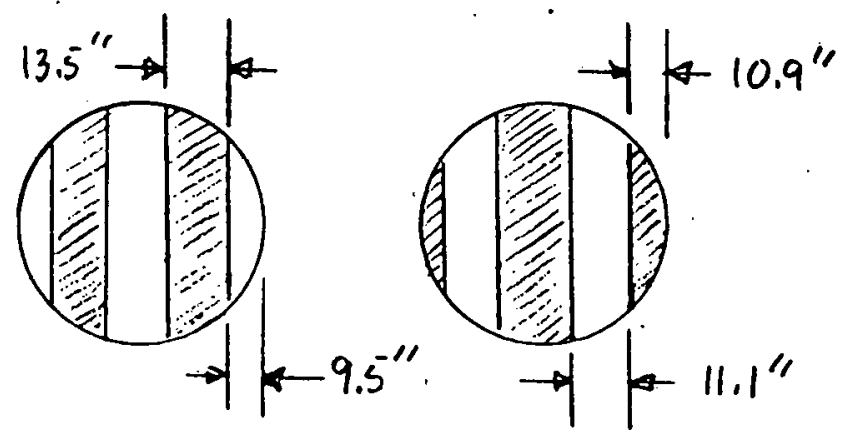




Assigned : 114
Case No. $\quad$.

\begin{tabular}{|c|c|c|c|c|}
\hline Type & $\begin{array}{l}\text { TEMA Exchanger } \\
\square \text { Special Exchanger }\end{array}$ & $\begin{array}{l}\text { Designation } A E L \\
\text { (Describe) }\end{array}$ & $\begin{array}{l}\text { Shell Orientation } \\
\text { NOT GIVEN }\end{array}$ & $\begin{array}{l}\text { Q Horizontal } \\
\text { Q Vertical }\end{array}$ \\
\hline
\end{tabular}

SHELL GEOMETRY (Complete Sketch Below)

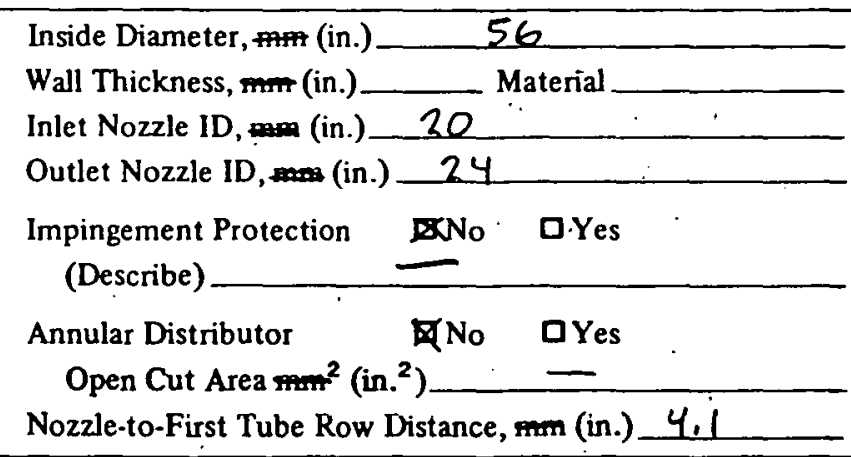

\section{CROSS BAFFLE GEOMETRY}

Type $\square$ Segmental; R Double-Segmental $\square$ Disc/Doughnut (OOUBCE)

$\square$ Triple-Segmental; $\square$ No-Tubes-in-Window

Baffle Cut, \% Shell Diameter 30

Cut Orientation Relative to Axis of Inlet Nozzle

Inlet Baffle $\square$ Perpendicular $\otimes$ Parallel $\square 45^{\circ}$

Central Baffles $\square$ Perpendicular Parallel $\square 45^{\circ}$

Baffle Thickness, (in.) 0.625 Material

Diametral Clearances Shell-to-baffle, (in.) 0,268

Tube-to-baffle (in.) 0.0156

Bundle-to-shell, (in.) 0.653

Number of Baffles Along Length of Shell 19

Baffle Spacing, (in.) _ Central 30 Inlet 28 Outlet 28

Unsupported Tube Span Lengths, (in.)

Longest 60 Inlet 58 Outlet $\_58$

\section{TUBE GEOMETRY}

Outside Diameter, (in.) $\quad 0.750$

Wall Thickness, $\mathrm{mm}$ (in.) 0,083 Material CS

Tube Lengths

Straight Tube, Inside Tubesheets, (in.) 596

U-Tube, Tubesheet to Bend Tangent, (in.)

Tube Pitch, mm (in.) $\quad 0.9375$

Layout (Please Circle)

Flow

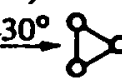

No. of Tubes 2910

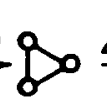

No. of Tubepasses

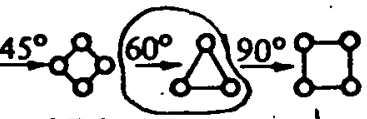

First Tubepass Countercurrent aCocurrent

Tube-to-Tubesheet Joint

$\square$ Welded $\square$ Roller Expanded $\square$ Other NOT G(UEN)

If U-Tube

Maximum Bend Radius, mm(in.)

Bend Orientation Relative to Axis of Shellside Inlet Nozzle

$\square$ Perpendicular $\square$ Parallel

If Bend Supported, Describe in Comments Below

If Finned Tubes

Finm (Fins/in.) _ _ Fin Material _ -

Diameter, (in.), Root — Over Fins -

If Enhanced Surface Tubes

(Describe)

\section{DETUNING BAFFLE}

If Detuning Baffle Used to Control Acoustic

Vibration, Indicate Position on Sketch Below

\section{COMMENTS AND SKETCH}

Complete sketches by drawing in tubeside and shellside nozzles.

Indicate inlet nozzles with an arrow.

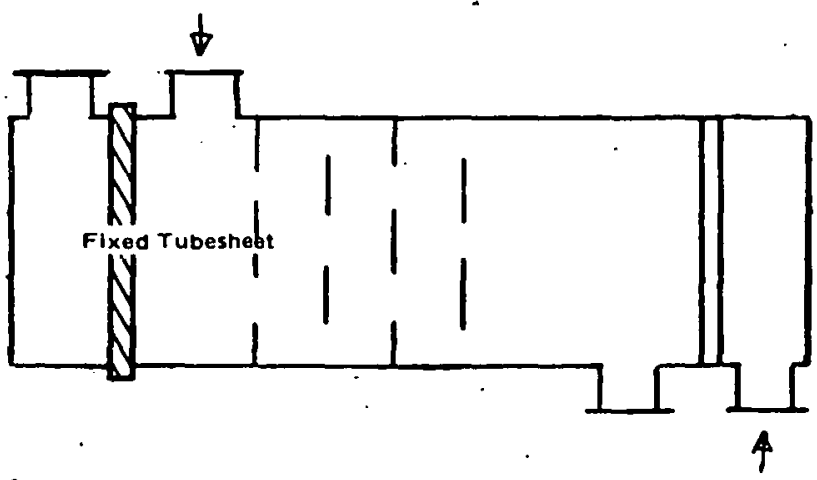

Snow shellslde inlet nozzle location, baffle cut orlentation, and Impingement devices.

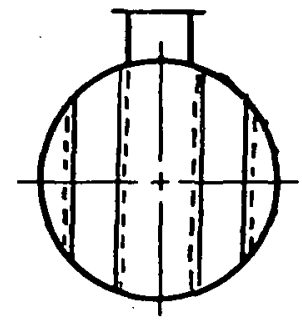

$\leadsto$ indicate top of exchanger as mounted 


\section{PROCESS CONDITIONS}

\begin{tabular}{|c|c|c|c|c|c|}
\hline & & \multicolumn{2}{|c|}{ Reference Condition A } & \multicolumn{2}{|c|}{ Reference Condition B } \\
\hline & & Tubeside & Shellside & Tubeside & Shellside \\
\hline Flow Rate, $\mathrm{k}\left(10^{3} \mathrm{lb} / \mathrm{hr}\right)$ & & 571.7 & 488.2 & & \\
\hline Inlet Temperature, $\in(F)$ & & 80 & -35 & & \\
\hline Outlet Temperature, $€(\mathrm{~F})$ & & -16 & 74 & & \\
\hline Inlet Pressure, $* \mathrm{P}_{a}$ (psia) Absolute & 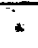 & 448 & 426 & & \\
\hline \multicolumn{6}{|l|}{ Measured $\Delta \mathrm{P}, \mathrm{kP}$-(psi) } \\
\hline Inlet Weight Fraction Vapor & & 1.0 & 1.0 & & \\
\hline Outlet Weight Fraction Vapor & & 1.0 & 1.0 & & \\
\hline Vibration Observed & & \multicolumn{2}{|c|}{ ONo XYes } & \multicolumn{2}{|c|}{ QYes } \\
\hline
\end{tabular}

\section{FLUID PHYSICAL PROPERTIES}

\begin{tabular}{|c|c|c|c|c|}
\hline Fill In All Applicable Entries & \multicolumn{2}{|c|}{ Tubeside } & \multicolumn{2}{|c|}{ Shellside } \\
\hline Fluid Name & \multicolumn{2}{|c|}{ RICH GAS } & \multicolumn{2}{|l|}{ LEAN } \\
\hline Reference Temperature $€(F)$ & 19.5 & 74 & -15.5 & 80 \\
\hline \multicolumn{5}{|l|}{$\begin{array}{l}\text { Liquid Properties at Reference } \\
\text { Temperatures }\end{array}$} \\
\hline \multicolumn{5}{|l|}{ Density, $\mathrm{kg} / \mathrm{m}^{3}\left(\mathrm{lh} / \mathrm{ft}^{3}\right)$} \\
\hline \multicolumn{5}{|l|}{ Viscosity, $\mathrm{mPa} \cdot \mathrm{s}(\mathrm{cP})$} \\
\hline \multicolumn{5}{|l|}{ Thermal Conductivity, $\mathrm{W} / \mathrm{m} \cdot \mathrm{C}(\mathrm{Btu} / \mathrm{m} \mathrm{ft} \mathrm{F})$} \\
\hline \multicolumn{5}{|l|}{ Heat Capacity, $\mathrm{kJ} / \mathrm{kg} \cdot \mathrm{C}(\mathrm{Btu} / \mathrm{m} \mathrm{ft} \mathrm{F})$} \\
\hline \multicolumn{5}{|l|}{$\begin{array}{l}\text { Vapor or Gas Properties at } \\
\text { Reference Temperatures }\end{array}$} \\
\hline \multicolumn{5}{|l|}{ Density, $\mathrm{kg} / \mathrm{m}^{3}\left(\mathrm{lb} / \mathrm{ft}^{3}\right)$} \\
\hline Viscosity, & 0.011 & 0.012 & 0,0108 & 0.0120 \\
\hline Thermal Conductivity, W/mre (Btu/hr ft F) & 0.0184 & 0.0206 & 0.0172 & 0.0207 \\
\hline Heat Capacity, kJ/kg-G-(Btu/lb F) & - & 0.585 & - & 0.604 \\
\hline Fluid Molecular Weight, k (lb/mole) & \multicolumn{2}{|c|}{16.62} & \multicolumn{2}{|c|}{17.41} \\
\hline \multicolumn{5}{|l|}{ If Boiling or Condensing } \\
\hline Latent Heat, k//kg (Btu/lb) & & - & & \\
\hline
\end{tabular}




\begin{tabular}{|l|}
\hline Assigned \\
Case No. 114 \\
\hline
\end{tabular}

\section{VIBRATION AND DAMAGE DESCRIPTION'}

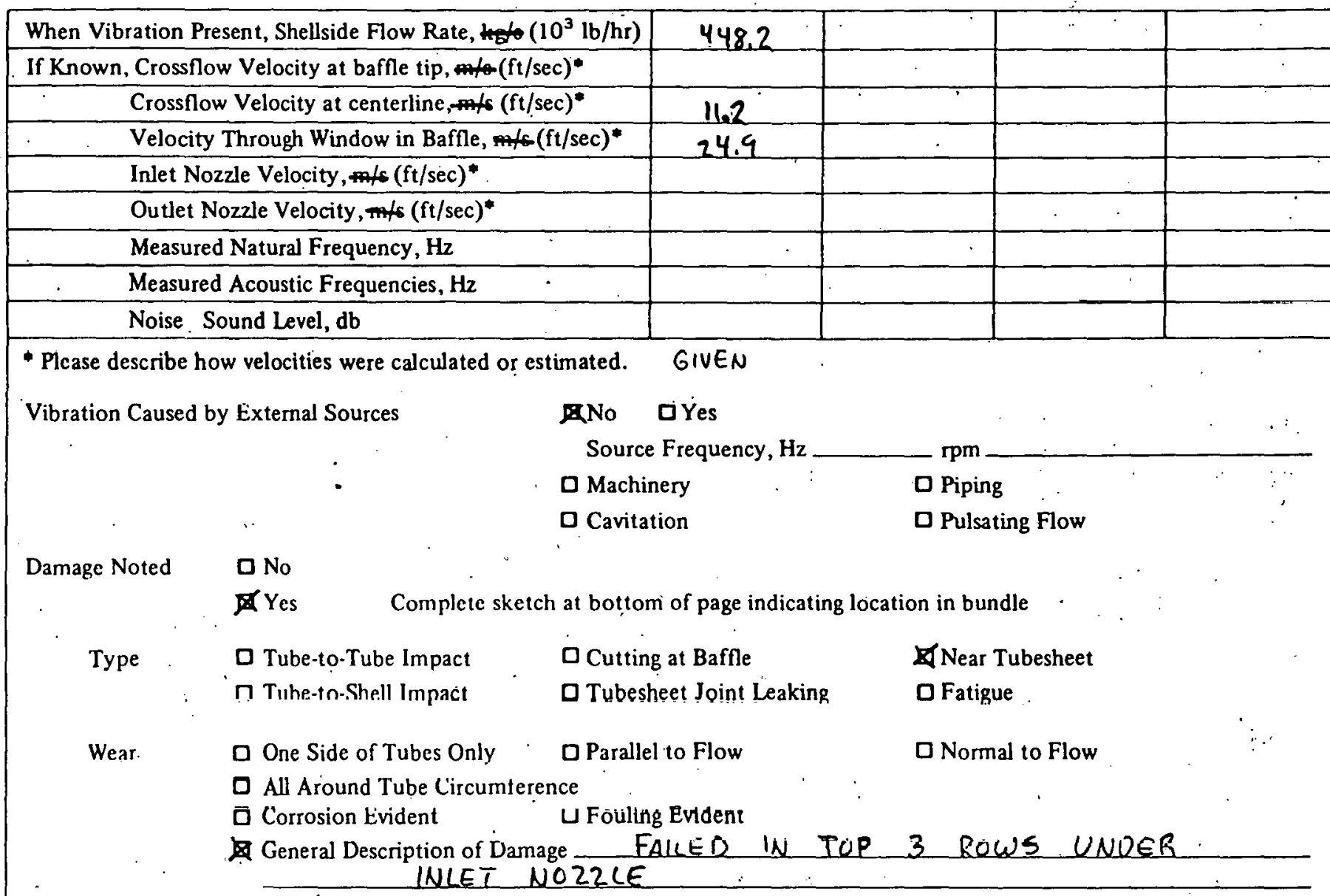

Exchanger Operation History

- How Long on Stream Before Damage Occurred?

- Any Unusual Occurrence Observed Prior to Vibration as a Consequence of
口Start-up
$\square$ Plant-Upset
Q Shutdown

Describe

- If Vibration Remedy Applied, Describe and Indicate Results:

TUBE BUNDLE DAMAGE SKETCH
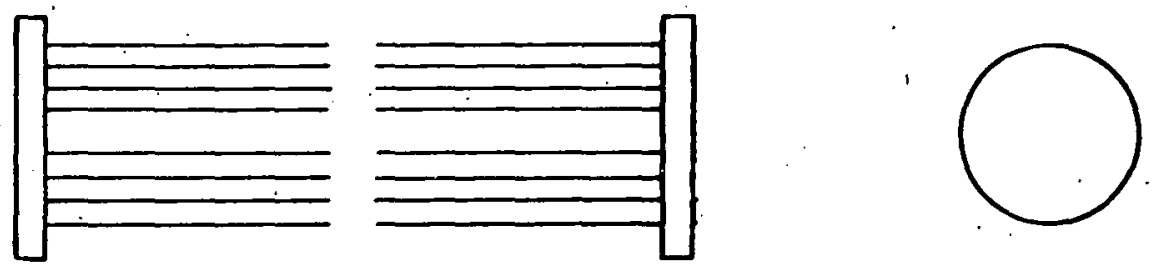
To protect the identity of the organization submitting this case, HTRI has assigned a case number. Additionally the data on pages 2, 3, and 4 have been reviewed to ensure that they do not include any propietary information. This is a replacement for the original page 1 that provides space for

Assigned

Case No. additional comments, drawings, photographs, etc.

\section{Summary}

This 69-in. diameter by 20-ft long BJS TEMA style heat exchanger is used as a process gas cooler. Shellside flow entered the single 42-in. nozzle at the center of the exchanger and was directed to the two ends by a solid flow divider plate. A portion of the nozzle was blanked off to prevent by passing and reduced the flow area to the equivalent of a 40.6 -in. diameter nozzle. There were five crosspasses on each end with segmental baffles. The outlet nozzles were 26 in. diameter and mated matching 26-in. diameter nozzles on a vessel that acted as a collecting header to combine the flow into a 42 in. diameter outlet line. See sketch - below. It should be noted that the tubefield layout was inline. There has not been any tube damage reported.. However, there is an extremely loud noise. The noise was greatest at the fixed tubesheet at the top of the shell and at the bottom of the shell near the floating head. The shellside pressure drop was pulsing between 2 to $4 \mathrm{psi}$. It is not clear that the noise was flowinduced inside the shell and not being generated by an outside source.

For this case, in addition to the data form, there are complete construction drawings of the exchanger (not the collecting header), a description of noise observations, and several sketches.

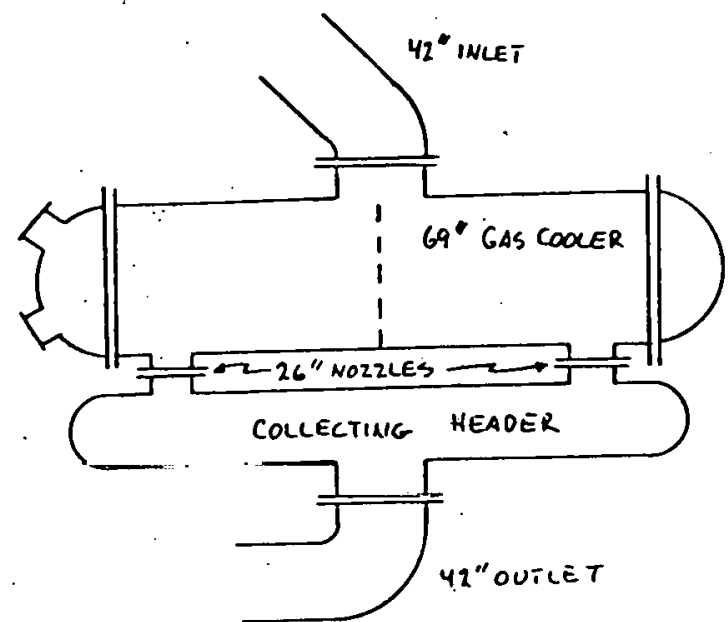




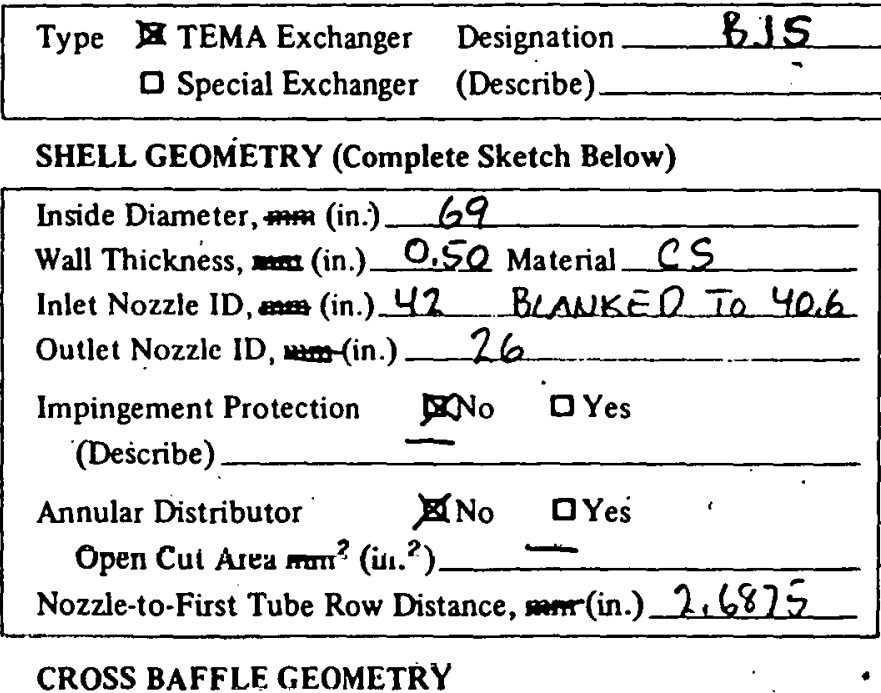

Type Segmental; 口Double-Segmental D Disc/Doughnut

$\square$ Triple-Segmental; $\square$ No-Tubes-in-Window

Baffle Cut, \% Shell Diameter 45

Cut Orientation Relative to Axis of Inlet Nozzle

Inlet Baffle $\$$ Perpendicular $\square$ Parallel $045^{\circ}$

Central Baffles $\square$ Perpendicular $\square$ Parallel $\square 45^{\circ}$

Baffle Thickness, (in.) 0.50 Material CS

Diametral Clearances Shell-to-baffle, (in.)

Tube-to-baffle (in.)

Bundle-to-shell, (in.)

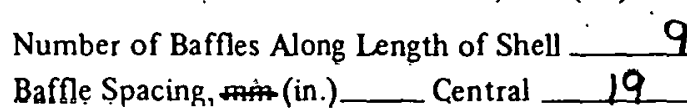

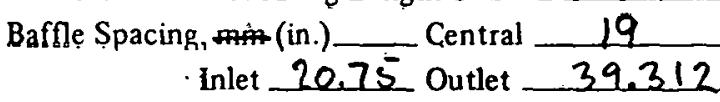

Unsupported Tube Span Lengths, (in.)

Longest 58.312 nnlet 38 Outlet 58.312

Shell Orientation $\mathbf{X}$ Horizontal

DVertical

\section{TUBE GEOMETRY}

Outside Diameter, $\operatorname{mm}$ (in.) 0.750

Wall Thickness, nm (in.) 0.083 Material CS

Tube lengths

Straight Tubc, Inside Tubesheets, (in.) 234.9

U-Tube, Tubesheet to Bend Tangent, man (in.) _-

Tube Pitch, (in.) 1.25

Layout (Please Circle)

No. of Tubes 2028

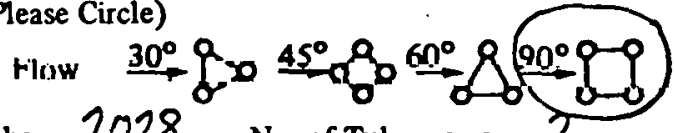

First Tubepass KCounterourrent $\mathrm{K}$ Cocurrent

Tube-to-Tubesheet Joint

DWelded $\mathbb{R}$ Roller Expanded $\square$ Other

If U-Tube

Maximum Bend Radius, mm (in.) ___

Bend Orientation Relative to Axis of Shellside Inlet Nozzle

$\square$ Perpendicular $\square$ Parallel

If Bend Supported, Describe in Comments Below

If Finned Tubes

Eimotm (Fins/in.) _- Fin Material __ -

Diameter, (in.), Roót $=$ Over Fins

If Enhanced Surface Tubes.

(Describe)

\section{DETUNING BAFFLE}

If Detuning Baffle Used to Control Acoustic

Vibration, Indicate Position on Sketch Below

\section{COMMENTS AND SKETCH}

Complete sketches by drawing in tubeslde and shellside nozzles.

indicate inlet nozzles with an arrow.

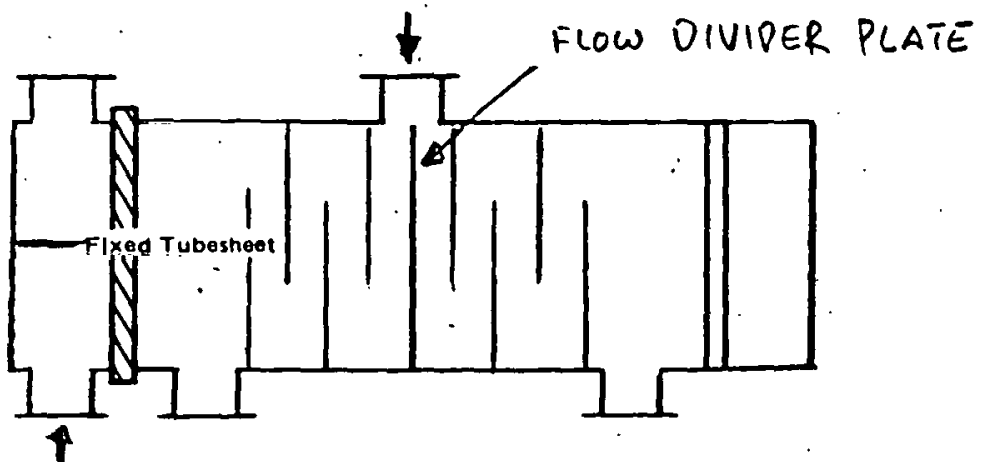

Show shellside Inlet nozzle locatlon, batfle cut orientation, and Impingement devices. 


\section{DOE/ANL/HTRI HEAT EXCHANGER TUBE VIBRATION DATA FORM}

Page 3

\begin{tabular}{l} 
Assigned \\
Case No. 115 \\
\hline
\end{tabular}

\section{PROCESS CONDITIONS}

\begin{tabular}{|c|c|c|c|c|}
\hline & \multicolumn{2}{|c|}{ Reference Condition A } & \multicolumn{2}{|c|}{ Reference Condition B } \\
\hline Flow Rate, $\left(10^{3} \mathrm{lb} / \mathrm{hr}\right)$ & $1697 *$ & 372 & & \\
\hline Inlet Temperature, $\mathrm{e}(\mathrm{F})$ & 87 & 192 & & \\
\hline Outlet Temperature, $\in(F)$ & 96 & 105 & & \\
\hline Inlet Pressure, 190 (psia) Absolute & 50 & 51 & & \\
\hline Inlet Weight Fraction Vapor & 0 & 1.0 & & \\
\hline Outlet Weight Fraction Vapor & 0 & 1.0 & & \\
\hline Vibration Observed & \multicolumn{2}{|l|}{ ONo } & \multicolumn{2}{|c|}{ QYes } \\
\hline
\end{tabular}

\section{CALCULATED}

\section{FLUID PHYSICAL PROPERTIES}

\begin{tabular}{|c|c|c|c|}
\hline Fịll In All Applicable Entries & Tubeside & \multicolumn{2}{|c|}{ Shellside. } \\
\hline Fluid Name & WATER & PROCESS & GAS \\
\hline Reference Temperaturc $+(\mathrm{F})$ & & 105 & 192 \\
\hline $\begin{array}{l}\text { Liquid Properties at Reférence } \\
\text { Temperatures }\end{array}$ & & & \\
\hline Density, $\mathrm{m}^{3}\left(\mathrm{lb} / \mathrm{ft}^{3}\right)$ & & & \\
\hline Viscosity, (cP) & & & \\
\hline Thermal Conductivity, W/mE (Btu/m ft F) & & & \\
\hline Heat Capacity, $\mathrm{kJ} / \mathrm{kg} \in(\mathrm{Btu} / \mathrm{m} \mathrm{ft} \mathrm{F})$ & & & \\
\hline $\begin{array}{l}\text { Vapor or Gas Properties at } \\
\text { Reference Temperatures }\end{array}$ & & & \\
\hline Density, $\mathrm{k} / \mathrm{m}^{3}\left(\mathrm{lb} / \mathrm{ft}^{3}\right)$ & & & \\
\hline Viscosity, mPaet (cP) & & 0.0099 & 0.0115 \\
\hline Thermal Conductivity, W/ma (Btu/hr ft F) & & 0.0366 & 0.0397 \\
\hline Heat Capacity, $\mathrm{kJ} / \mathrm{kg} \mathrm{C}-(\mathrm{Btu} / \mathrm{lb} \mathrm{F})$ & & 0.3330 & 0.6090 \\
\hline Fluid Molecular Weight, $\mathrm{kg} / \mathrm{lb} / \mathrm{mole})$ & & 22 & 59 \\
\hline If Boiling or Condensing & & & \\
\hline Latent Heat, $/$ kg (Btu/lb) & & $\ldots$ & \\
\hline
\end{tabular}




\section{VIBRATION AND DAMAGE DESCRIPTION}

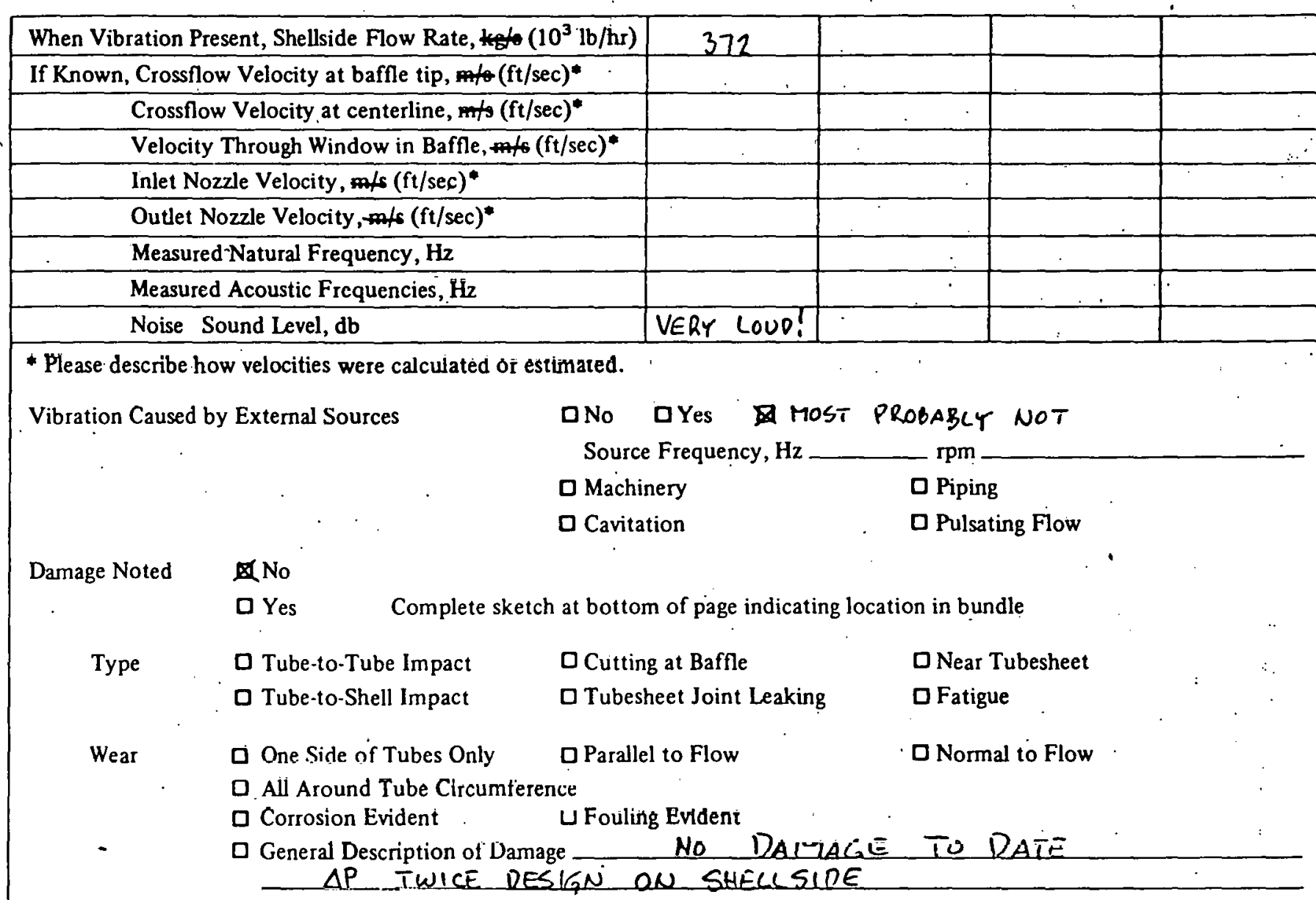

Exchanger Operation History

- How Long on Stream Before Damage Occurred?

- Any Unusual Occurrence Observed Prior to Vibration as a Consequence of
DStart-up
Q Plant-Upset
$\square$ Shutdown.

Descrihe

- If Vibration Remedy Applied, Describe and Indicate Results:

\section{TUBE BUNDLE DAMAGE SKETCH}
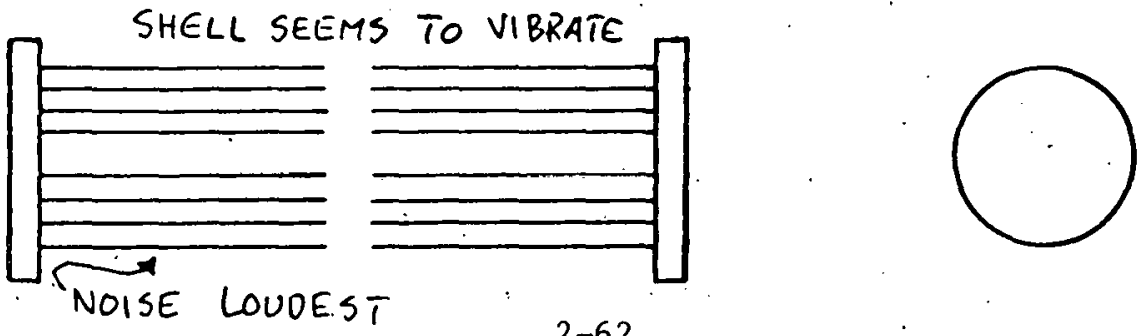